\title{
A NOVEL HYBRID PHOTOACOUSTIC-FLUORESCENT CONTRAST AGENT TO TRACK AQUEOUS HUMOUR PATHWAYS IN VIVO
}

By

Kirsten Cardinell

B.Sc. Ryerson University, Toronto, Ontario, 2017

\author{
A thesis \\ presented to Ryerson University \\ in partial fulfillment of the \\ requirements for the degree of \\ Master of Science \\ in the program of Biomedical Physics
}

Toronto, Ontario, Canada, 2019

(C) Kirsten Cardinell, 2019 


\section{AUTHOR'S DECLARATION}

I hereby declare that I am the sole author of this thesis. This is a true copy of the thesis, including any required final revisions, as accepted by my examiners.

I authorize Ryerson University to lend this thesis to other institutions or individuals for the purpose of scholarly research.

I further authorize Ryerson University to reproduce this thesis by photocopying or by other means, in total or in part, at the request of other institutions or individuals for the purpose of scholarly research.

I understand that my thesis may be made electronically available to the public.

Kirsten Cardinell 


\title{
Abstract
}

\section{A NOVEL HYBRID PHOTOACOUSTIC-FLUORESCENT CONTRAST AGENT TO TRACK AQUEOUS HUMOUR PATHWAYS IN VIVO}

\author{
Kirsten Cardinell \\ Master of Science, Biomedical Physics \\ Department of Physics, Ryerson University , 2019
}

Contrast agents are frequently used in photoacoustic imaging (PAI) to study the lymphatic system. However, there is a lack of standardized approaches to evaluate agents for specific applications. Furthermore, validation of PAI results through post-mortem fluorescence imaging is often required. To address this, hybrid modality contrast agents would be advantageous. Using unbiased characterization experiments and analyses three commercial NIR dyes are compared: IRDye QC-1, IRDye800CW, and CF770 for the minimum detectable concentration, linear range, and stability of PA signal with prolonged laser exposure. QC-1 had the most desirable properties for quantitative lymphatic PAI specifically for glaucoma treatment testing. Therefore a hybrid tracer was developed and QC-1 alongside fluorescent dye BODIPY was labelled to bovine serum albumin (QBB). QBB tracer showed nearly identical PA properties to free QC-1 dye and maintained a strong fluorescent signal after laser exposure. The QBB tracer shows promise as a hybrid contrast agent for in vivo lymphatic PAI. 


\section{Acknowledgements}

First and foremost, I would like to thank my supervisors Dr. Yeni Yucel, Dr. Carl Kumaradas, and Dr. Neeru Gupta. I consider myself extremely lucky to have had the privilege of working with Dr. Yucel on several projects throughout my undergraduate and graduate studies. I was given the opportunity in my $3^{\text {rd }}$ year of my undergraduate degree to join his lab as a co-op student. Since then, Dr. Yucel has consistently provided me with valuable knowledge, guidance, and opportunities that have been essential to my growth and success as a researcher. His door was always open whenever I had questions or ran into problems, and his passion for this project helped make these past two years productive and enjoyable. Dr. Yucel is a visionary researcher and I hope to have more opportunities to work with him in the future.

My sincere thanks to Dr. Kumaradas for being my co-supervisor, enabling me to do this project. Dr. Kumaradas was always happy to provide me with assistance and direction whenever I needed it and I greatly appreciate his contributions in my committee meetings and towards this thesis document.

Dr. Gupta, although not an official supervisor, has always provided me with valuable feedback on my work as well as clinical background knowledge essential for experimental design. She always found ways to build upon and improve my work whether it be through experiments, presentations, or document writing. Dr.Gupta's insights are incredibly valued, and I am lucky to have access to her expertise in the field of Glaucoma research.

I would like to thank my supervisory committee members Dr. Michael Kolios and Dr. Bryan Koivisto. Dr. Kolios was my very first mentor and greatly contributed to my development as a researcher. He introduced me to photoacoustic imaging and I have never looked back since. Dr. Kolios' scientific input during my committee meetings were extremely helpful for the development and progression of my thesis project.

Dr. Koivisto's excitement of this project and contribution towards the development of a hybrid photoacoustic-fluorescent contrast agent made this thesis a pleasure to work on. I greatly appreciate the time 
he took to help me with my knowledge in chemistry and his expertise was heavily relied on through to the completion of this thesis.

I am grateful to Dr. Toronov for agreeing to be my external examiner and for his careful input and guidance aimed to improve the quality of my thesis document.

I would also like to express my gratitude to all the Gupta \& Yucel lab members and St. Michael's staff that have aided in my research ambitions. Xun Zhou contributed to this project by providing training on experimental protocols, contributing to the experimental designs and set up, and aiding in the interpretation of data. Xun also performed all of the mouse eye injections and dissections discussed in the future directions portion of this thesis. I was also very lucky to have Megan Yuen and Amanda Mac preparing and sectioning the mouse tissue for me, and I am grateful for Joseph Hanna who graciously took the time out of his busy schedule to train me on the protocol for tissue staining. Both Joseph Hanna and Caterina Di Ciano-Oliveira were helpful with training me to use the confocal microscope to image said tissue sections. I would also like to thank Hyacinth Irving, who performed and provided expertise on the statistical analysis of the data. A special thanks to Dr. Paola Luciani for her contribution as a coauthor to the manuscript entitled "An Approach to Test the Suitability of a Commercial Near-Infrared Dye for Quantitative In Vivo Photoacoustic Lymphatic Imaging" that makes up Chapter 2 of this thesis document.

Last, but not least, I would like to thank my parents for their love and support, my sister, Jillian, for her tolerance of my shenanigans, and the rest of my family for all their encouragement. I would also like to thank my friends and peers for providing an escape from our studies and making the last two years a blast. Finally, I would like to thank Nico Arezza for everything he did to help me during these past two years, whether it be reading over my work, or providing support.

Thank you all for your contributions to my experience in this master's program. Without you, this accomplishment would not have been possible. 


\section{Table of Contents}

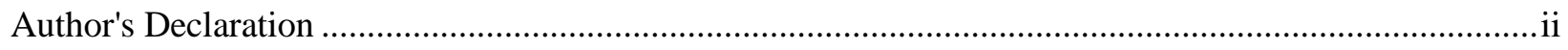

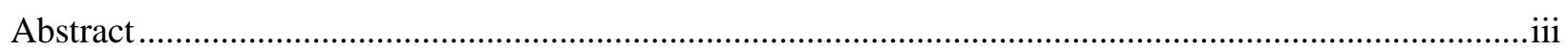

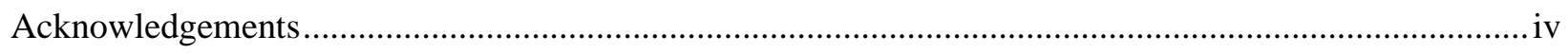

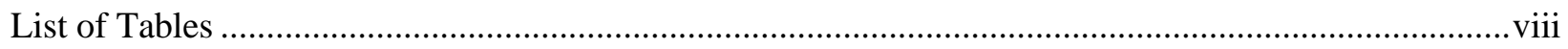

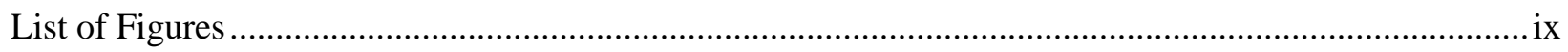

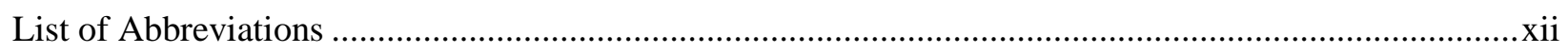

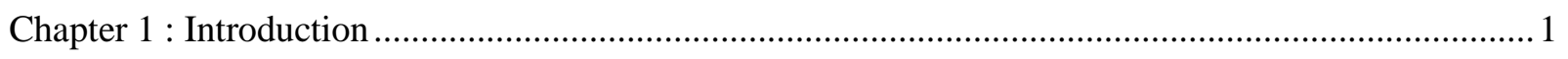

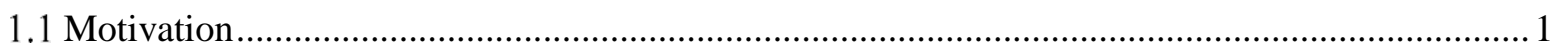

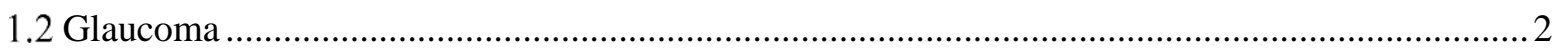

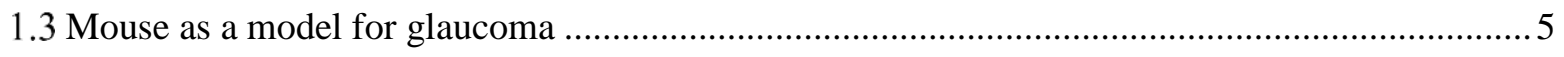

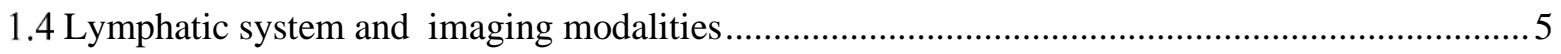

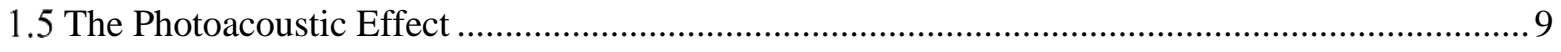

1.6 Contrast Agents for Photoacoustic Imaging …............................................................... 12

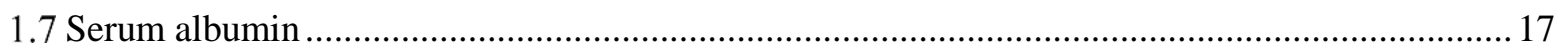

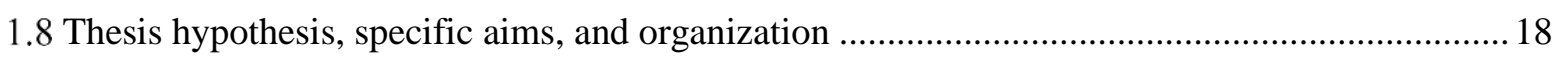

Chapter 2: An Approach to Test the Suitability of a Commercial Near-Infrared Dye for Quantitative In

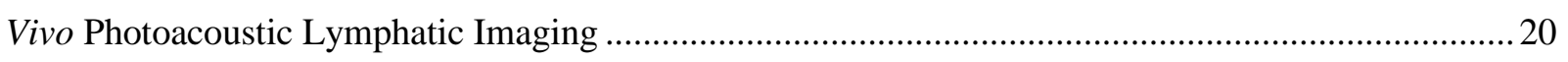

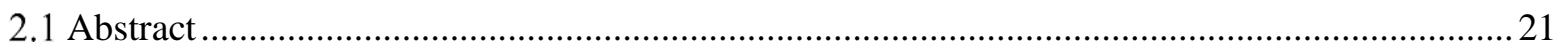

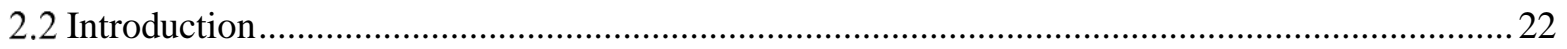

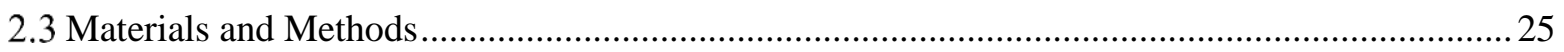


2.4 Results

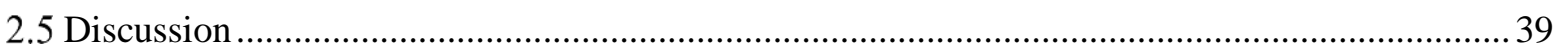

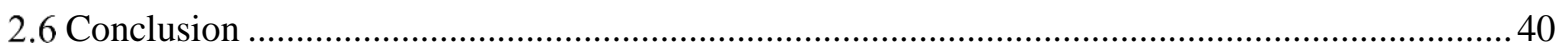

Chapter 3 : A Hybrid Tracer for Photoacoustic and Fluorescence Imaging of Lymphatic Drainage from

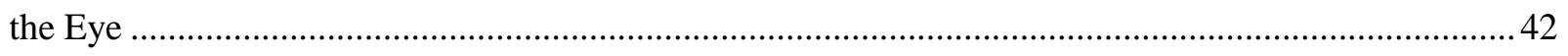

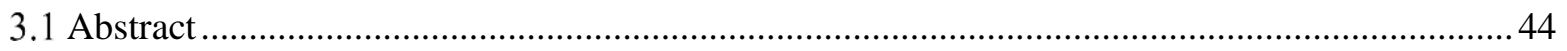

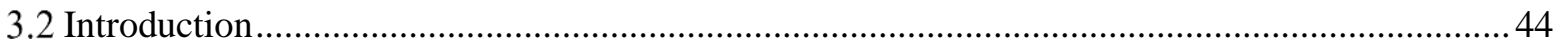

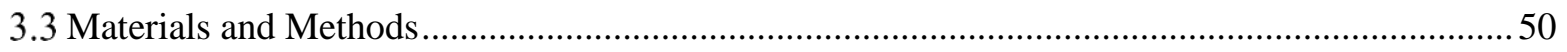

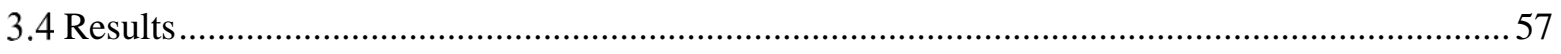

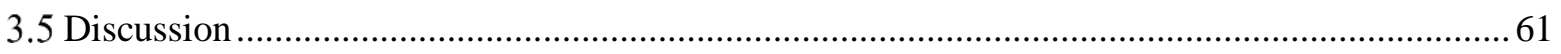

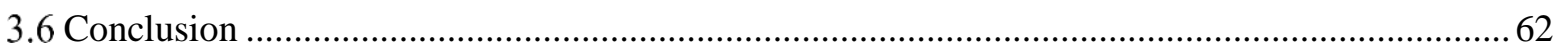

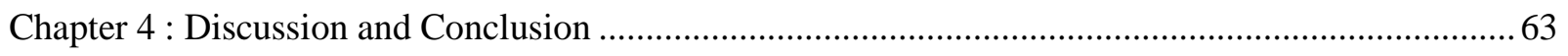

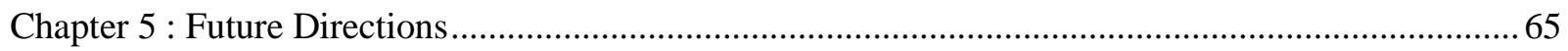

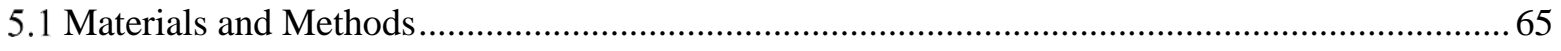

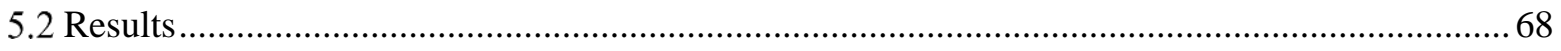

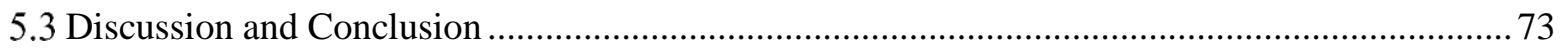

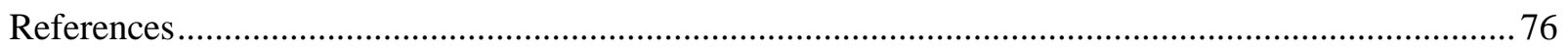




\section{List of Tables}

Table 1. The list of NIR dyes used in this study along with their maximum absorption wavelength

$\left(\lambda_{\max \text { abs }}\right)$, molar extinction coefficient $(\varepsilon)$, cost per $0.5 \mathrm{mg}$, and supplier.

Table 2. Minimum detectable concentration: The estimated minimum concentration detectable in vitro by

PA imaging for NIR dyes. .36

Table 3. Linear range: The approximate ranges between two concentrations where the photoacoustic signal intensity is linearly related to the concentration.

Table 4. Properties of commonly use fluorescent dyes with peak emission wavelengths below the imaging range of the MSOT and the quenching range of QC-1

Table 5. Fluorescence count reading of QBB with $480 \mathrm{~nm}$ excitation and $520 \mathrm{~nm}$ emission. Values are the average of 3 samples. .61 


\section{List of Figures}

Figure 1-1. Labelled eye diagram. ${ }^{9}$

Figure 1-2. Normal eye (left) compared to eye with glaucoma (right). Images designed based off of diagrams in Weinreb et al. ${ }^{4}$

Figure 1-3. Diagram of the lymphatic system and blood circulation. The capillary bed shifts from red to blue indicating deoxygenation of hemoglobin in blood. Artery and vein are indicated in red and blue, respectively. The high pressure of the capillaries forces blood plasma out into the surrounding tissue (black arrows) which is taken up by the lymphatic vessels (green). The fluid is subsequently filtered by the lymph nodes (not shown) ${ }^{38}$ 6

Figure 1-4. Simplified diagram of photoacoustic imaging of a light absorber within a phantom. 10

Figure 1-5. A simplified Jablonski diagram contrasting the difference between fluorescent and nonfluorescent dye excitation and return to ground state. 15

Figure 1-6. Structure of human serum albumin. ${ }^{95}$. 17

Figure 2-1. Phantom preparation and imaging set up. A) Diagram of a home-made phantom. B) Phantom holder and PA multispectral tomography animal imaging system (MSOT) diagram. C) Reconstructed PA image of the dye imaging set up. The red circle shows the dye region of interest, and the blue circle shows the control (PBS) region of interest. The intersect of dotted lines indicate the center of the transducer ring shown in B. The scale bar indicates 2mm. Diagrams A) and B) are approximated and not to scale..........29 Figure 2-2. Near-infrared dye molar extinction coefficients and absorption spectra measured from 680 to 980 nm. A) Molar extinction coefficients determined from the spectrophotometer measurements. B) PA mean pixel intensity (MPI) or PA spectra. Concentration of each dye: QC-1 $(24.06 \mu \mathrm{M}), \mathrm{CF} 770(26.55 \mu \mathrm{M})$, IRDye 800CW $(23.33 \mu \mathrm{M})$, all diluted in PBS. .33

Figure 2-3. PA signal (mean pixel intensity, MPI) as a function of dye concentration for all near-infrared dyes. A) Concentration ranges from 0 to approximately $200 \mu \mathrm{M}$. B) Concentration ranges from 0 to 
approximately $10 \mu \mathrm{M}$. Error bars represent the standard deviations of MPI between the imaging slices in the three samples 34

Figure 2-4. Minimum Detectable Concentration and PA signal (mean pixel intensity, MPI) as a function of dye concentration. Concentration ranges from $0 \mu \mathrm{M}$ to approximately $25 \mu \mathrm{M}$. A) IRDye QC-1 B) CF770 C) IRDye 800CW. Limit of Detection line shown in black. MDC for each dye is: QC-1 (0.000992 a.u.), CF770 (0.000367 a.u.), and IRDye 800CW (0.00033 a.u.). PBS MPI is shown in gray. Error bars represent the standard deviations of MPI between the imaging slices in the three samples. .35

Figure 2-5. The line of best fit for the statistically linear range of concentrations for each dye. Bars represent the $95 \%$ confidence interval. .37

Figure 2-6. The normalized PA signal (mean pixel intensity, MPI) as a function of imaging time (total 1 hour) for QC-1 (red), CF770 (orange), and IRDye 800CW (blue). Error bars represent the standard deviation between the three imaging sessions of three separate dye samples. .38

Figure 3-1. General structure of BODIPY $: \mathrm{Z}, \mathrm{Y}=\beta$ or 2,6 positions, $\mathrm{X}=$ meso position, $\mathrm{W}=\alpha$ or 3,5 positions, $\mathrm{V}=1,7$ positions or other $\beta$ positions. Pentamethyl BODIPY is $\mathrm{Z}, \mathrm{Y}=-\mathrm{H} ; \mathrm{X}, \mathrm{V}, \mathrm{W}=$ methyl. 48 Figure 3-2. The normalized absorbance spectrum of A) free QC-1 (purple) and BODIPY (blue), single dye bound BSA (orange and red respectively), and the QBB tracer (green), and B) The QBB tracer alone. Semitransparent purple is the quenching range of QC-1 and semi-transparent red is the imaging range of the MSOT PA imager. .58

Figure 3-3. The linear range. The range of concentrations where the relationship between the QBB concentration (determined by the concentration of QC-1) and the photoacoustic detection is statistically linear. Error bars the indicate the standard deviation of the three trials. A.U. arbitrary unit. .59 Figure 3-4. The graphical representation of the minimum detectable concentration for the QBB tracer. The green data points represent the average mean pixel intensity of the photoacoustic signal of QBB. Error bars are the standard deviation. The black line is the limit of detection, and the green line is the line of best fit. The intersection between the green and black lines is the minimum detectable concentration (1.22 $\mu \mathrm{M})$. A.U. arbitrary unit. 
Figure 3-5. The average photoacoustic signal of QBB over time of laser irradiation at $735 \mathrm{~nm}$. Error bars represent the standard deviation between the three samples of QBB.

Figure 5-1. Photoacoustic images of the mouse head cross-section at the region of the eyes after injection of QBB tracer. The top row is mouse M1, the second row is M2, and the third row is M3. Green represents the photoacoustic signal generated from the QBB tracer. $S=$ Superior, $\mathrm{R}=$ Right, $\mathrm{L}=$ Left, $\mathrm{I}=$ Inferior. Scale bar $=5 \mathrm{~mm}$. The vertical bar on the right shows pixel intensity values of the QBB tracer ranging from 0 to 2800

a.u.

Figure 5-2. Photoacoustic images of the mouse head cross-section at the region of the neck and cervical lymph nodes after injection of QBB tracer. The top row is mouse M1, the second row is M2, and the third row is M3. Green indicates the QBB tracer signal. $\mathrm{S}=$ Superior, $\mathrm{R}=$ Right, $\mathrm{L}=\mathrm{Left}, \mathrm{I}=$ Inferior. Scale bar $=5 \mathrm{~mm}$. Asterisks indicate the location of right cervical lymph node without tracer signal. Arrows indicate the presence of tracer signal in right cervical lymph node. The vertical bar on the right shows pixel intensity values of the QBB tracer ranging from 0 to 740 a.u. .70

Figure 5-3. Infrared (nm) images of mice heads (top row) and necks (bottom row) with blue autofluorescence $(\mathrm{nm})$ images overlaid in green. The green indicates the tracer in the right eye in all three mice. $\mathrm{R}=\mathrm{Right}, \mathrm{L}$ $=$ Left. The arrowheads indicate the location of the cervical lymph nodes. M1, M2, and M3 are the mice ID numbers. .71

Figure 5-4. Right (injected) eye (A) and left (non-injected) eye (B) histology slide sections of mouse \# M2. Green is the tracer, blue is the nuclear stain. $\mathrm{N}=$ Nasal and $\mathrm{T}=$ Temporal. The scale bar is $600 \mu \mathrm{m} . \ldots . . .72$ Figure 5-5. Right (A) and left (B) cervical lymph nodes histology slide sections of the mice M2 under confocal laser microscopy. Green is the tracer, blue is the nuclear counterstain with DAPI. No green tracer is noted in both cervical lymph nodes. SG = salivary gland, arrowheads point to lymph node border. The scale bar indicates $200 \mu \mathrm{m}$. 73 


\section{List of Abbreviations}

\begin{tabular}{|c|c|}
\hline $\mathrm{AH}$ & Aqueous Humour \\
\hline BSA & Bovine Serum Albumin \\
\hline CEUS & Contrast-Enhanced Ultrasound \\
\hline DCE-MRI & Dynamic Contrast-Enhanced MRI \\
\hline $\mathrm{DMF}$ & N, N-Dimethylformamide \\
\hline DNA & Deoxyribonucleic acid \\
\hline FDA & US Food and Drug Administration \\
\hline HSA & Human Serum Albumin \\
\hline ICG & Indocyanine Green \\
\hline IOP & Intraocular Pressure \\
\hline LOB & Limit of the Blank \\
\hline LOD & Limit of Detection \\
\hline LOQ & Limit of Quantification \\
\hline MDC & Minimum Detectable Concentration \\
\hline MPI & Mean Pixel Intensity \\
\hline MRI & Magnetic Resonance Imaging \\
\hline MSOT & Multispectral Optoacoustic Tomography \\
\hline NIR & Near-Infrared \\
\hline PA & Photoacoustic \\
\hline PAI & Photoacoustic Imaging \\
\hline PAT & Photoacoustic Tomography \\
\hline PBS & Phosphate Buffer Saline \\
\hline PET & Positron Emission Tomography \\
\hline QBB & QC-1/BSA/BODIPY \\
\hline
\end{tabular}


ROI

RPM
Region of Interest

Rotations per minute 


\section{Chapter 1 : Introduction}

\subsection{Motivation}

Photoacoustic imaging (PAI) is a three-dimensional (3D) hybrid imaging modality combining optical acoustic imaging. ${ }^{1}$ PAI reconstructs images from ultrasonic pressure waves induced by the absorption of pulsed laser light by various molecules in the tissue. Laser light in the near-infrared (NIR) range from 650 to $950 \mathrm{~nm}$ is used to achieve a greater penetration depth due to lower tissue absorption in this range. ${ }^{2}$ Unique wavelength dependent, light-absorbing properties of the chromophores in tissue enables the detection and differentiation between endogenous chromophores such as melanin, oxygenated and deoxygenated hemoglobin through a deconvolution method called spectral unmixing. For biological processes or entities that do not produce a photoacoustic signal (do not possess chromophores that absorb light in the NIR wavelength range), exogenous chromophores are used as contrast agents to increase the signal to noise ratio at the targeted tissues or processes. Currently, there are no standardized approaches for characterizing and comparing potential contrast agents for use in specific experimental conditions or photoacoustic imaging systems. Quantification analysis should be performed on new contrast agents to test for characteristics such as the minimum detectable concentration, range of concentrations for linear analysis, and stability of photoacoustic signal with increasing laser exposure. Since biological processes assessed with in vivo photoacoustic imaging requires computational methods for image reconstruction and spectral unmixing, validation of PAI results is required by another imaging modality, for example through fluorescence imaging and histology, or else the results will be met with skepticism from the scientific community. Therefore, suitable contrast agents need to be developed for the dual purpose of in vivo photoacoustic imaging and post-mortem validation using fluorescence in situ imaging and microscopy. Additionally, a non-biased, systematic method of characterizing the contrast agents is required to determine its PAI properties to determine their suitability as contrast agents for various experimental conditions. 


\subsection{Glaucoma}

Glaucoma is the world's leading cause of irreversible blindness. ${ }^{3}$ It is characterized by the degeneration of retinal ganglion cells, and is often associated with high intraocular pressure (IOP), a major risk factor. ${ }^{3-5}$ IOP is the fluid pressure within the eye and is maintained through the production and drainage of an intraocular clear fluid called aqueous humour. Therefore, high IOP is caused by an increased production of aqueous humour, a decrease of aqueous humour drainage from the eye, or a combination of both. The only proven method of treatment for glaucoma is the lowering of IOP. ${ }^{4}$ This is achieved by using either medication or surgery depending on the type of glaucoma.

Aqueous humour fills the anterior and posterior chambers of the eye (Figure 1-1). It consists primarily of water, electrolytes, carbohydrates, urea, antioxidants, peptides and proteins, oxygen, and carbon dioxide. ${ }^{6}$ The proteins in aqueous humour include albumin, immunoglobulins, growth factors, and angiogenic regulators ${ }^{7}$, which increase with aging. ${ }^{8}$ Furthermore, the aqueous humour is verly low in protein with 200 times less protein than that in plasma. ${ }^{6}$

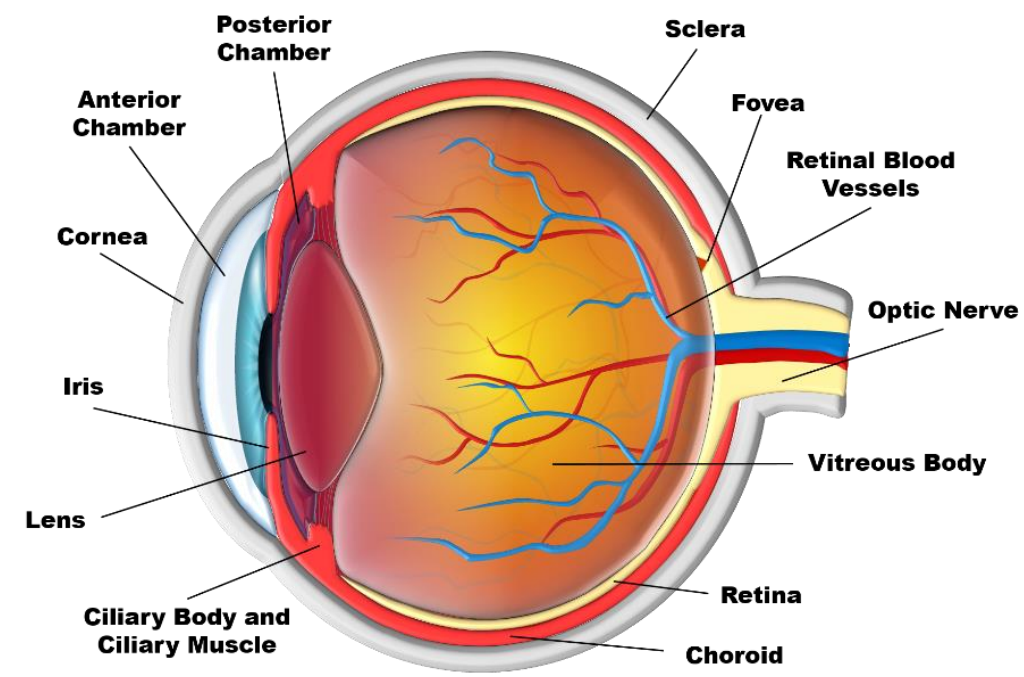

Figure 1-1. Labelled eye diagram..$^{9}$ 
Aqueous humour $(\mathrm{AH})$ is produced within the ciliary processes located in a region within the ciliary body just posterior to the iris. ${ }^{6}$ Diffusion, ultrafiltration, and active secretion are the mechanisms involved in $\mathrm{AH}$ production. Diffusion occurs when solutes, primarily sodium chloride, are transferred through tissue membranes located between the capillaries and the posterior chamber and ultrafiltration is the movement of water and water-soluble substances into the ciliary stroma. ${ }^{6,10}$ While diffusion and ultrafiltration are passive processes, secretion of molecules requires energy and it is mediated by protein transporters within the cellular membrane..$^{6,11}$

There are several AH drainage pathways from the eye. The conventional pathway was the first pathway discovered where the AH passes through the trabecular meshwork, and collector channels and aqueous veins, prior to entering the venous system via the episcleral veins. The unconventional, or uveoscleral pathway routes $\mathrm{AH}$ through the iris root into the ciliary body, choroid, and sclera. ${ }^{12,13}$ The uveolymphatic pathway is a newly discovered pathway where AH exits the eye through ciliary body lymphatics. The presence of this pathway has been demonstrated through the finding of ciliary body lymphatic vessels in human eye sections,${ }^{14}$ the detection of intracamerally injected radioactive and fluorescent tracers in the ciliary body lymphatics and cervical lymph nodes in sheep and mice respectively, ${ }^{15,}{ }^{16}$ and the dynamic tracking of intracamerally injected near-infrared dye-labelled albumin from the eye to the cervical lymph nodes in mice. ${ }^{17}$

There are two main types of glaucoma: closed-angle and open-angle (Figure 1-2). In closed-angle glaucoma, the closure of the anterior chamber angle between the iris and cornea blocks the drainage pathways. ${ }^{5}$ Closed-angle glaucoma makes up one-third of patients with primary glaucoma worldwide, especially in Asian populations ${ }^{18}$ and is associated with more immediate signs and symptoms and can be considered an ocular emergency when the condition is acute and requires urgent treatment. ${ }^{19}$ Open-angle glaucoma shows no apparent obstruction of the AH drainage, but the drainage pathways are functionally and structurally impaired, often resulting in increased IOP, a major risk factor. ${ }^{4,19}$ 


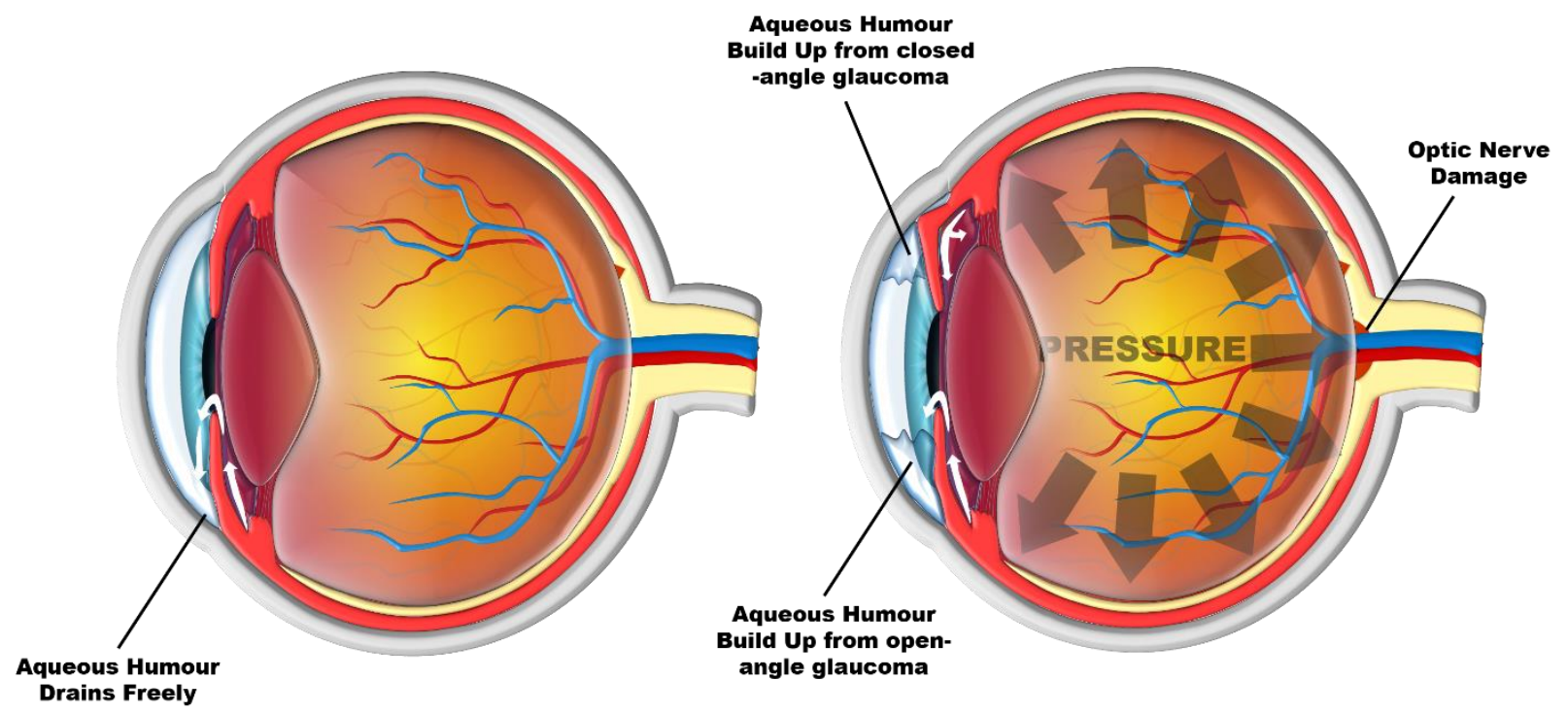

Figure 1-2. Normal eye (left) compared to eye with glaucoma (right). Images designed based off of diagrams in Weinreb et al. ${ }^{4}$

Although glaucoma cannot be cured, its progression towards blindness can be slowed or halted through IOP-lowering treatment. The first line of treatment is the use of topical medications. The fewest possible medications with the fewest adverse side effects are used to achieve the targeted IOP. ${ }^{20}$ When medications fail to achieve sufficient IOP reduction, surgical procedures such as laser or microsurgery techniques, such as trabeculectomy, are used. Trabeculectomy is the most common incisional surgical procedure as it is more effective than other surgical options. ${ }^{21}$ Trabeculectomy creates a partial excision of the trabecular meshwork or the corneoscleral tissue to create an alternative drainage pathway of the $\mathrm{AH}$ into the subconjunctival space.

The most widely used topical medication is Latanoprost, a prostaglandin $\mathrm{F}$ 2alpha analogue. ${ }^{22}$ Having previously been known for increasing uveoscleral drainage, ${ }^{23}$ a study using quantum dots discovered that Latanoprost increases $\mathrm{AH}$ from the eye also via the uveolymphatic pathway where the tracer signal increased in the cervical lymph node sections of the treated mouse group. ${ }^{20}$ However, the quantification of the tracer signal could be performed only in post-mortem tissue sections. By developing a non-invasive, in vivo method to dynamically and quantitatively track the AH through the uveolymphatic drainage pathway, 
we can test novel glaucoma drugs by comparing the rate of lymphatic drainage between treated and nontreated animals in preclinical studies.

\subsection{Mouse as a model for glaucoma}

There are many similarities between the mouse ocular anatomy and physiology and that seen in humans. Mice have similar AH dynamics in terms of intraocular pressure (10 to $20 \mathrm{mmHg}$ in mice and $16 \mathrm{mmHg}$ in humans), episcleral venous pressure, and turnover rate. ${ }^{25}$ Additionally, mice have similar anatomical structure, outflow pathways, and responses to IOP-lowering glaucoma drugs. ${ }^{26,27}$

Mice are cost-effective and easy to breed which allows for large sample sizes. They also have a considerable advantage over other species for genetic manipulation studies which enable the study of various physiological processes such as development ${ }^{28}$ and aging ${ }^{29}$ as well as human diseases. There are several experimentally induced mouse models for glaucoma ${ }^{30}$ and naturally-occurring inherited models of glaucoma (DBA/2J) mice are well-studied and frequently used. ${ }^{31,32}$ This makes the mouse an ideal model for studying lymphatic drainage from the eye and its role in glaucoma and glaucoma treatments.

\subsection{Lymphatic system and imaging modalities}

The lymphatic system is composed of lymphatic vessels, lymph nodes, and other lymph organs distributed throughout the body. It forms an integrated network to maintain fluid homeostasis, aid in the absorption of fat from the digestive system, and to maintain immunocompetence. ${ }^{33}$ Pressure and concentration gradients cause fluid to exit the blood vessel capillaries and enter the interstitial space as interstitial fluid which is then taken up by lymphatic vessels. The lymph is a clear fluid that does not contain any chromophores or other molecules that can be detected using any imaging modality to date. Furthermore, the lymph contains only approximately half the amount of proteins found in plasma. ${ }^{34}$ Therefore, all current imaging methods for lymphatics in the visible and near-infrared range require injected exogenous contrast agents. 
The lymphatic vessels also contribute to the metastasis of several cancers such as breast cancer, melanoma, and head and neck cancers. ${ }^{35}$ It also contributes to inflammation ${ }^{36}$, diabetes ${ }^{34}$, and obesity. ${ }^{37}$ Furthermore, due to the recent discovery of the uveolymphatic drainage pathway of the eye $\mathrm{e}^{14,15}$, lymphatics can also be relevant in glaucoma and its treatment. Therefore, lymphatic imaging is an important means of diagnosing and monitoring diseases, as well as developing novel treatments. There are many methods of lymphatic imaging and they will be briefly described in the following sections.

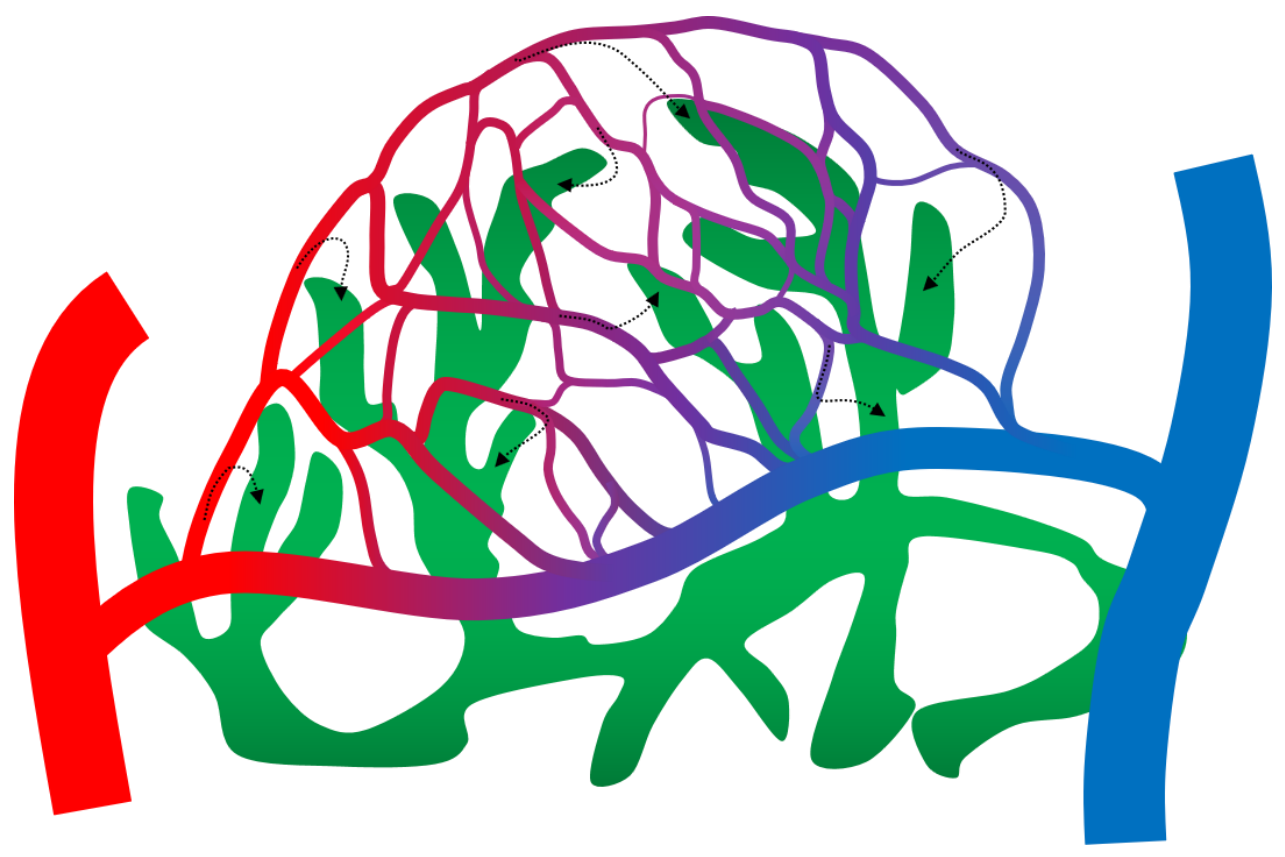

Figure 1-3. Diagram of the lymphatic system and blood circulation. The capillary bed shifts from red to blue indicating deoxygenation of hemoglobin in blood. Artery and vein are indicated in red and blue, respectively. The high pressure of the capillaries forces blood plasma out into the surrounding tissue (black arrows) which is taken up by the lymphatic vessels (green). The fluid is subsequently filtered by the lymph nodes (not shown). ${ }^{38}$

\subsubsection{Conventional Lymphatic Imaging}

In lymphangiography, poppy seed oil conjugated to iodine is injected into the lymph vessels in the lower limbs. ${ }^{39,40}$ Several consecutive X-rays or CT scans can depict the anatomy and the function of lymphatic flow. Lymphangiography is rarely used diagnostically today due to its invasiveness, cost, and risk of complication. ${ }^{38}$ 
Lymphoscintigraphy is a branch of nuclear medicine used to image the lymphatic system. Lymphoscintigraphy requires the introduction of a gamma-emitting radioactive tracer (technetium-99m conjugated sulfur colloid or albumin) that can then be detected using a gamma camera to measure and locate the radiation emitted from the tracer. ${ }^{38,41}$ This method was first discovered in 1953 and is currently the gold standard for lymphatic imaging. ${ }^{42}$

Despite its frequent use for measuring lymph drainage and lymphedema quantification, ${ }^{43}$ lymphoscintigraphy requires radiation exposure -albeit minuscule amounts- and has a relatively poor resolution. An alternate imaging solution without the use of radiation is required to reduce risks to patients.

\subsubsection{Fluorescence Optical Lymphatic Imaging}

Fluorescence optical imaging uses light to excite fluorescent molecules to generate fluorescence signal in tissue. The autofluorescence of the tissue and/or fluorescence of an exogenous contrast agent can then be detected. Autofluorescence is a broad, featureless spectrum that fluoresces across the visible and NIR wavelengths. The intensity of autofluorescence varies with the tissue type and the presence of the autofluorescence can lead to high background signal and negatively affect the detection of fluorescent labels. ${ }^{44}$ Additionally, autofluorescence is less prominent in the NIR range. ${ }^{45}$ Fluorescence optical imaging also has limited imaging depth due to light scattering and absorption by tissue. To reduce this limitation, emission light wavelengths in the near-infrared $(700 \mathrm{~nm}-900 \mathrm{~nm})$ are selected for as blood absorbs the least in this region allowing for a greater imaging depth. However, this still only achieves a maximum imaging depth of 5 to $7 \mathrm{~mm} .^{46}$

\subsubsection{Computed Tomography (CT)}

CT imaging provides accurate lymph node detection when using contrast agents. CT imaging with Iohexol, a water-soluble non-ionic monometric iodine contrast agent, was able to identify sentinel lymph nodes in a preliminary study using a tongue VX2 carcinoma model. ${ }^{47}$ 
However, due to the spatial resolution required for flow imaging, it is difficult to track the flow of lymph using CT imaging. Furthermore, the use of ionizing radiation for CT imaging may be cause for concern when repetitive imaging is required.

\subsubsection{Magnetic resonance imaging (MRI)}

MRI is able to detect the enlargement of lymph nodes. ${ }^{38}$ More recently, a method called dynamic contrastenhanced MRI (DCE-MRI) technique was developed to measure lymph flow and lymphatic dysfunctions through the mapping of lymph drainage in mice. ${ }^{48}$ DCE-MRI uses multiple MRI scans after the intravenous injection of a contrast agent.

Lymphotropic nanoparticle-enhanced MRI often uses iron oxide nanoparticles which adds cell-specific (malignant vs benign cellular content) information in addition to conventional MRI with the aim to provide improved assessment of lymph nodes. ${ }^{40}$

MRI is an often-sought-after imaging modality for its penetration depth, and lack of radiation exposure. However, MRI is a large and expensive imaging modality with a relatively large resolution $(\sim 1 \mathrm{~mm})^{49}$ compared to other imaging modalities such as optical coherence tomography $(\sim 2-10 \mu \mathrm{m})^{50}$, PAI $(\sim 100 \mu \mathrm{m}$ depending on the transducer $)^{51}$, and ultrasound $(\sim 150 \mu \mathrm{m}$ depending on the transducer $) .^{49} \mathrm{~A}$ cheaper and portable method of lymph flow analysis would be more feasible and allow for easily accessible lymphatic analysis.

\subsubsection{Ultrasound}

Contrast-enhanced ultrasound (CEUS) is a promising imaging modality for detecting sentinel lymph nodes due to its low cost and high signal-to-noise ratio. ${ }^{52} \mathrm{~A}$ sentinel lymph node is typically the first lymph node or group of lymph nodes that cancer reaches when a tumor metastasizes. Microbubbles are the most commonly used ultrasound contrast agents and can be made with a variety of materials; however, their large size (less than $\sim 10 \mu \mathrm{m}$, approximately the size of a red blood cell), results in several caveats such as slow 
uptake. Furthermore, CEUS has poor spatial resolution and the imaging of the lymph is dependent on the operator's sonography experience..$^{53}$

\subsubsection{Positron emission tomography (PET)}

PET is currently the most sensitive in vivo technique for imaging molecular pathways in humans ${ }^{54}$. PET radioactive tracers are physiologically and pharmacologically relevant compounds labeled with positronemitting isotopes such as fluoride-18 or carbon-11. After being either injected or inhaled by a patient, the location and quantity of the tracer are assessed with a PET scanner. The sensitivity and specificity of PET is the major strength of this technique. However, PET scans involve radioactive exposure, although minimal, it limits the number of scans a patient should undergo and proves a risk during pregnancy, as is with other imaging modalities requiring the use of ionizing radiation. Additionally, it is a costly procedure.

\subsubsection{Photoacoustic imaging (PAI)}

PAI is a three-dimensional (3D) hybrid imaging modality combining the optical and acoustic imaging. ${ }^{1} \mathrm{PAI}$ allows for in vivo functional imaging by utilizing light absorbing properties of chromophores by irradiating them with laser lights of various wavelengths. A laser light used to generate the photoacoustic signal is limited by the depth it can reach in tissue; however, the acoustic pressure waves generated by the light absorption can travel much further resulting in a larger penetration depth for PAI compared to purely optical imaging modalities. Several studies have used PAI for lymphatic imaging by using a wide variety of

contrast agent. ${ }^{55-58}$ Photoacoustic imaging does not use ionizing radiation and is a relatively cheap and portable imaging modality.

\subsection{The Photoacoustic Effect}

The photoacoustic (PA) effect was first discovered in 1880 by Alexander Graham Bell. ${ }^{59}$ When lightabsorbing molecules called chromophores are illuminated with pulses of laser light, some of the energy from the absorbed light is converted into heat. This local temperature increase results in thermoelastic 
expansions which produce high-frequency pressure waves $(>20 \mathrm{kHz})$ within a medium and can be detected by ultrasound transducers (Figure 1-4). ${ }^{60}$

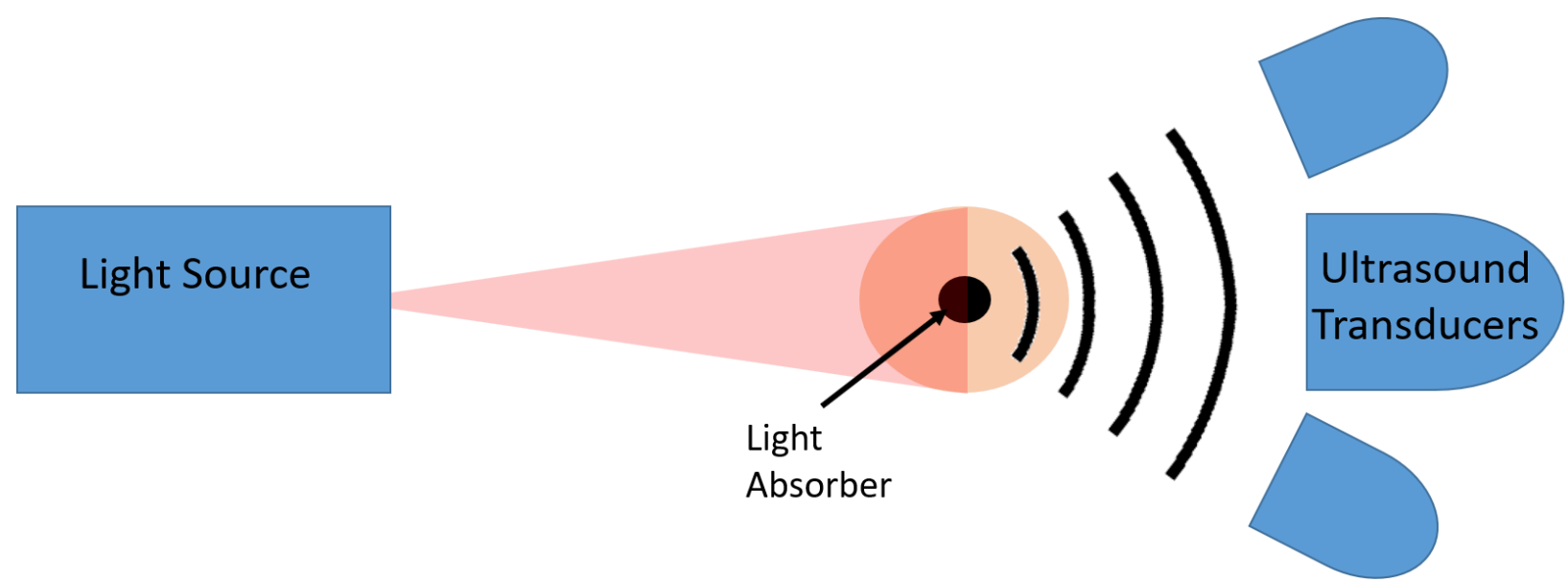

Figure 1-4. Simplified diagram of photoacoustic imaging of a light absorber within a phantom.

To use the PA effect for medical imaging, the duration (or width) of the laser pulse of the imaging system needs to satisfy the following conditions: 1$)$ the pulse must be shorter than the thermal relaxation time $\left(\tau_{t h}\right)$ so that heat diffusion is negligible throughout the laser pulse, and 2) the pulse must be shorter than the stress relaxation time $\left(\tau_{s}\right)$ so that the volume expansion within the matter is negligible throughout the laser pulse. ${ }^{60}$ These two constraints are defined by the following equations:

$$
\begin{gathered}
\tau_{t h}=\frac{d_{c}^{2}}{4 D_{T}} \\
\tau_{s}=\frac{d_{c}}{v_{s}}
\end{gathered}
$$

where $d_{c}$ is the dimension of the heated region, or, the absorber size, $D_{T}$ is the thermal diffusivity of the sample, and $v_{S}$ is the speed of sound. 
The initial pressure wave $(p)$ at position $r$ generated by thermoelastic expansion in an acoustically homogenous and non-viscous medium can then be calculated using the following equation so long as the previously mentioned conditions are met: ${ }^{1}$

$$
p(\vec{r})=\frac{\beta}{\rho C_{v} \kappa} \mu_{a} \phi(\vec{r})
$$

where $\beta$ is the isobaric volume expansion coefficient of the material $\left(\mathrm{K}^{-1}\right)$, and $C_{p}$ is the isobaric specific heat of the material $\left(\mathrm{J} \mathrm{K}^{-1} \mathrm{~kg}^{-1}\right), \mu_{a}$ and $\phi$ are the absorption coefficient and the optical radiation fluence rate of the material, respectively.

\subsubsection{Photoacoustic Spectral Unmixing}

Due to the dependence on light absorption for photoacoustic signal generation, the different light absorbance profiles of chromophores across multiple wavelengths enables their differentiation in PA images. Since the size of a chromophore is significantly smaller than the resolution of a tomographic PA device, it is assumed that there can be multiple types of chromophores within a single pixel. A deconvolution technique called spectral unmixing is used to determine the concentration (micromolars) of chromophores in each pixel based on the pixel spectrum. There are many different methods of spectral unmixing that fall under linear and non-linear methods. The most basic method of spectral unmixing assumes a linear relationship between the relative amount of chromophores within a pixel and the photoacoustic spectrum of the pixel and relationship is represented by the equation can be seen below ${ }^{61}$ :

$$
\mu_{a}(\lambda, r)=\sum_{i=1}^{n} \varepsilon_{i}(\lambda) C_{i}(r)+\eta(\lambda, r)
$$

Where $\mu_{a}$ is the absorption coefficient of a pixel at location $r$ over the imaged wavelengths, $\mathrm{n}$ is the number of chromophores, $\varepsilon_{i}$ is the molar extinction coefficient of chromophore $i, C_{i}$ is the concentration of 
chromophore $i$ at location $r$, and $\eta$ is the noise. Obtaining accurate concentrations of chromophores within a pixel can be determined by, but not limited to, least squares error analysis and various different combinations of constraints on the algorithm ${ }^{62}$. Furthermore, there are many algorithms used assuming a non-linear relationship between each chromophore concentration and the absorption coefficient of a pixel. ${ }^{63}$

Computer analysis of photoacoustic images using spectral unmixing algorithms is a useful tool for detecting exogenous contrast agents from the background signal generated by tissue. ${ }^{64}$ However, spectral unmixing faces challenges due to spectral colouring, noise, image reconstruction artifacts, laser power fluctuation, and animal movement which may lead to inaccurate results. ${ }^{61}$ For this reason, photoacoustic imaging of biological processes should be accompanied by rigourous validation techniques.

\subsection{Contrast Agents for Photoacoustic Imaging}

A contrast agent is a substance that improves the contrast of structures or fluids for better medical imaging quality by increasing the signal to noise ratio. In PAI, the acoustic signal detection is based on the optical absorption properties of two categories of chromophores: endogenous and exogenous. Endogenous chromophores are those that are already present in the body. Some examples include oxygenated and deoxygenated hemoglobin, melanin, and cytochrome oxidase. Although these endogenous chromophores do have applications, these applications are rather limited to primarily melanin in melanoma, and hemoglobin oxygenation in blood. ${ }^{65}$ Applications in other biological systems require exogenous chromophores which are introduced to the body. Exogenous chromophores can be added to increase the maximum imaging depth, locate biological entities and/ or observe physiological functions. An exogenous contrast agent for photoacoustic imaging is made of two primary components: a targeting ligand and a signaling compound.

Targeting ligands are selected based on their size and charge such that the desired biological entity and/or function is targeted. A targeting ligand may be a small molecule, peptide, affibody, aptamer, antibody, or protein. 
A signaling compound is based on the imaging modality requirements. For PAI, signaling compounds are generally selected to have a high optical absorbance (molar extinction coefficient) with a sharp peak within the NIR optical window (700 - $900 \mathrm{~nm}$ ), high photostability, low quantum yield, and efficient heat conversion. ${ }^{66}$ There are three categories of signaling compound: Organic nanostructures, metallic or semiconducting nanostructures, and NIR dyes. ${ }^{66,67}$

Metallic nanoparticles are a class of contrast agents for PAI due to their modifiable properties such as material type, particle shape, and size $(<1000 \mathrm{~nm})$, and surface properties such as charge and polarity. ${ }^{66}$ Metallic nanoparticles also have higher molar extinction coefficients than NIR dyes, therefore producing stronger photoacoustic signals. However, the ability to reproduce, purify, and quantify nanoparticles is challenging. ${ }^{68}$ Additionally, the most widely used material for metallic nanoparticles is gold, where its toxicity is still debated. ${ }^{69-73}$

Organic nanostructures primarily include conjugated polymers, porphyrin-based structures, and encapsulations. ${ }^{51}$ These nanostructures have high flexibility in terms of composition, shape, charge, and size. They can act as the contrast agents on their own, as well as enhance other contrast agents or signaling compounds. Furthermore, they can also be the foundation of activatable probes. The development of these types of contrast agents is still relatively new, and therefore more in-depth information on biocompatibility to demonstrate their potential for in vivo photoacoustic imaging is still needed..$^{51}$

NIR dyes tend to have relatively good tissue distribution and penetration, low toxicity, and fast clearance rates compared to the other two categories of signaling compounds. ${ }^{66}$ Furthermore, NIR dyes can be conjugated to various biologically relevant proteins and enzymes ${ }^{74}$ and due to their small sizes, there should be little to no change of the biological abilities of the protein or enzyme. This enables the development of tracers that can be used to track biodistribution and biological processes. We have performed this in previous work by tracking lymphatic drainage using NIR dye-labelled albumin ${ }^{17}$ which is the most abundant 
plasma protein found to have a high accumulation in the lymph nodes and is often used as a targeting ligand for lymphatic imaging. ${ }^{75}$

\subsubsection{Fluorescence vs Non-fluorescence}

There are two types of dyes: fluorescent, and non-fluorescent. When either type of dye absorbs light, the energy from the light excites the molecules from a ground state to an excited state. The amount of light energy absorbed is proportional to the molar extinction coefficient of the dye molecule. Once a molecule has been excited to a higher energy state, it will eventually return to the ground state. For fluorescent dyes, some energy will be given off through vibration producing heat, and some energy will be given off by producing a lower energy, longer wavelength photon (Stokes shift). A characteristic known as the quantum yield is the number of photons emitted compared to the number of photons absorbed. A non-fluorescent dye returns to ground state exclusively by vibrational energy and therefore has a quantum yield nearing zero. An example of a simplified Jablonski diagram to compare the two types of dyes is shown in Figure $1-5$.

Photoacoustic imaging relies on the production of heat to generate the thermoelastic expansion, therefore, a non-fluorescent dye should produce a stronger photoacoustic signal than a fluorescent dye with similar properties due to the lower quantum yield. ${ }^{51}$ However, even though a non-fluorescent dye should produce a stronger photoacoustic signal, they will not be detected by fluorescence validation imaging. 


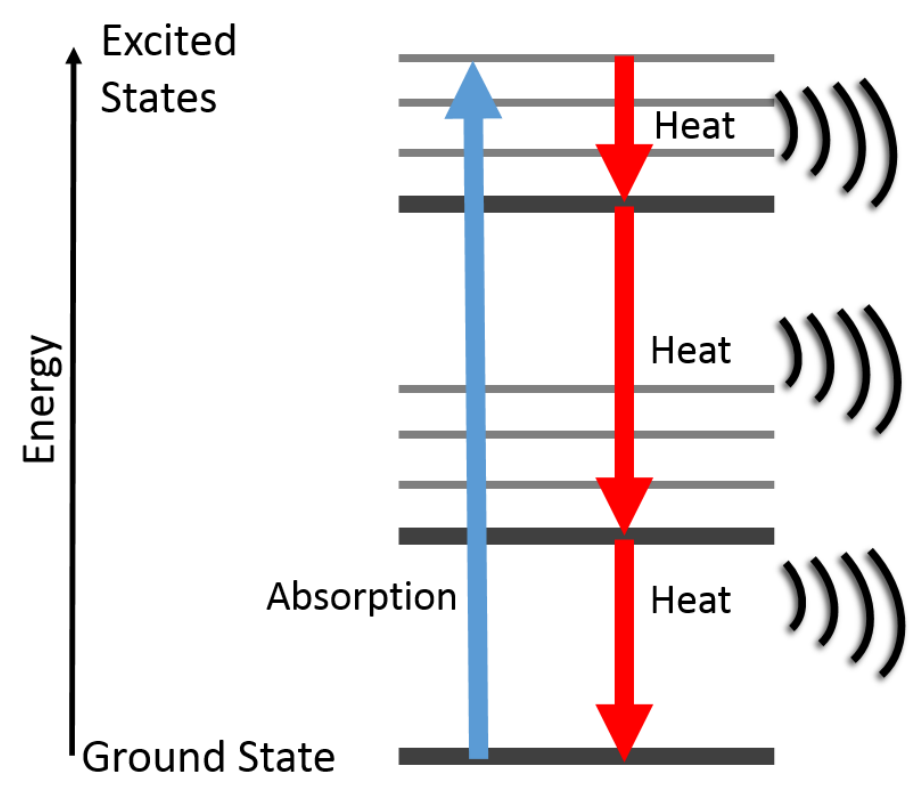

Non-Fluorescence

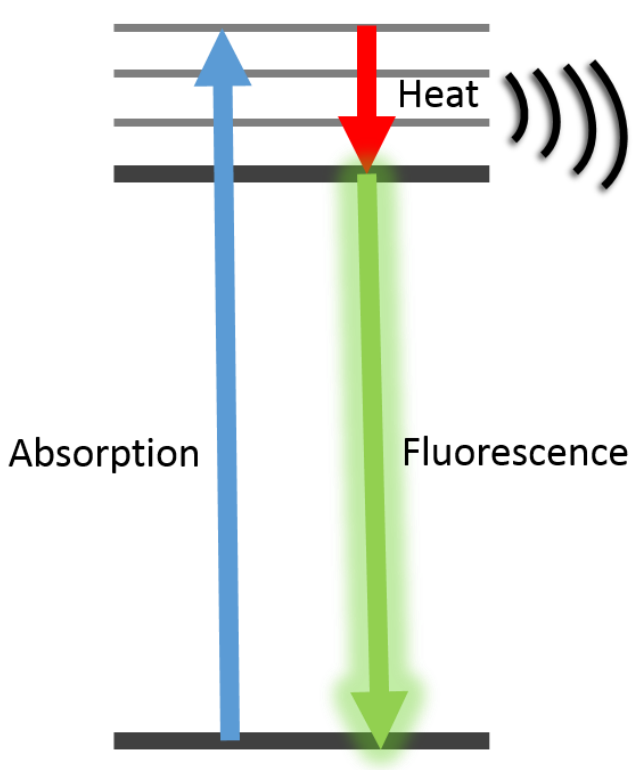

Fluorescence

Figure 1-5. A simplified Jablonski diagram contrasting the difference between fluorescent and non-fluorescent dye excitation and return to ground state.

\subsubsection{Commercially Available Near-infrared Dyes}

There is an extensive list of NIR fluorescent dyes available on the market and only a handful of NIR nonfluorescent dyes. For this thesis, the following commercial NIR dyes will be discussed: quencher dye IRDye QC-1, and fluorescent dyes CF770, IRDye 800CW, and Indocyanine green (ICG).

IRDye QC-1 (LI-COR Biosciences, NE, USA) is a dark (non-fluorescent), broad-range quencher dye designed to quench (absorb) the fluorescence signal for several common fluorophores such as IRDye 800CW, IRDye 700DX, Fluorescein, Cy3, and Cy5. ${ }^{76}$ QC-1 has a peak absorption at $737 \mathrm{~nm}$ in PBS with an extinction coefficient of $96,000 \mathrm{M}^{-1} \mathrm{~cm}^{-1}$ and a molecular weight of $1243.75 \mathrm{~g} / \mathrm{mol} .{ }^{76} \mathrm{QC}-1$ has been used as a signalling compound for photoacoustic imaging on several occasions including but not limited to, phantom studies. ${ }^{55,66}$ The dynamic tracking of aqueous humour to the lymph nodes ${ }^{16}$, and target-dependent PA signal detection via DNA aptamers. ${ }^{78}$ 
IRDye $800 \mathrm{CW}$ is a commonly used fluorescent dye. It has a molar extinction coefficient of $240,000 \mathrm{M}^{-1} \mathrm{~cm}^{-}$ 1, a peak absorption wavelength of $774 \mathrm{~nm}$, an emission maximum at $789 \mathrm{~nm}$, and a molecular weight of $1166.20 \mathrm{~g} / \mathrm{mol}$. IRDye $800 \mathrm{CW}$ has been used in various applications for fluorescence imaging ${ }^{79,80}$ as well as photoacoustic imaging. ${ }^{81-83}$

CF770 (Biotium, CA, USA) is a fluorescent dye with a molar extinction coefficient of $220,000 \mathrm{M}^{-1} \mathrm{~cm}^{-1}$ where the peak absorption wavelength is $770 \mathrm{~nm}$ which emits light at $797 \mathrm{~nm}$. Although CF770 absorbs light in the NIR window, it has not, to our knowledge, been used for photoacoustic imaging.

Indocyanine green (ICG) is the only FDA (US Food and Drug Administration) approved and clinically used NIR dye. After its introduction, it was quickly used for recording dye dilution curves, primarily for cardiac output. ICG has an absorption maximum near the isosbestic point of oxygenated and deoxygenated hemoglobin $(\sim 800 \mathrm{~nm})$, binds to plasma proteins after intravenous injection resulting in its confinement to the vascular system, has low toxicity and rapid excretion. ${ }^{84}$ However, ICG has poor stability after light exposure. ${ }^{85}$

\subsubsection{BODIPY}

BODIPY (4,4'-difluoro-4-bora-3a,4a-diaza-s-indacene) dyes are a family of fluorescent dyes that are strongly absorbing, emit relatively sharp fluorescence peaks, and have high quantum yields. They are also relatively insensitive to properties of their environment (the polarity and $\mathrm{pH}$ ) and are reasonably stable in physiological conditions. ${ }^{86}$ BODIPY dyes have also been used and developed for protein, DNA, and lipid labeling. ${ }^{86-89}$ BODIPY dyes are commercially available and can be selected for peak absorption wavelengths from $502 \mathrm{~nm}$ to $646 \mathrm{~nm} .{ }^{90}$ Since their peak absorption wavelengths operate outside the NIR window, BODIPY dyes have not been recommended for use for photoacoustic imaging. BODIPY is used for in situ and microscopic fluorescence imaging. 


\subsection{Serum albumin}

Human serum albumin (HSA, Figure 1-6) is the most common protein in plasma with a concentration of 3.5-5.0 g/dl. It is $66 \mathrm{kDa}$ in size with dimensions of 40 by 40 by $140 \mathrm{~A}^{\mathrm{o}} .^{91}$ Its primary functions are to maintain oncotic pressure and to transport molecules such as hormones and fatty acids. ${ }^{92}$ Serum albumin has been explored for its use in cancer chemotherapy as a drug carrier to promote tumor uptake and increased permeability and retention..$^{93,94}$

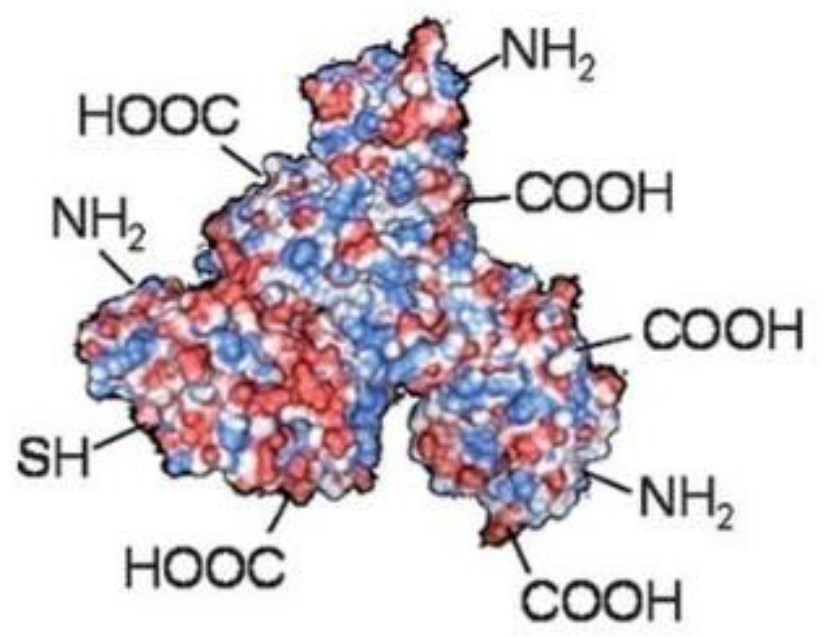

Figure 1-6. Structure of human serum albumin. ${ }^{95}$

HSA has several primary amines (-NH 2 , shown in Figure 1-6) which are positively charged at physiologic $\mathrm{pH}$ which therefore results in their occurrence on the outside surface of the HSA structure making them readily accessible for the conjugation to amine-reactive groups such as N-hydroxysuccinimide esters (NHS esters). HSA also contains hydrophobic regions ${ }^{96-98}$ which can be used as binding cites for phenoxyacetic and phenylacetic acids, and ligands. HSA also contains a relatively larger proportion of acidic amino acids making it negatively charged at physiological $\mathrm{pH} .{ }^{92}$

HSA is a promising carrier for cancer-targeted imaging and therapy. ${ }^{99}$ For photoacoustic imaging, HSA was bound to indocyanine green (ICG) for tumor targeting. ${ }^{100-103}$ HSA is also used for lymph node 
targeting ${ }^{75}$ and in our previous work, we used bovine serum albumin (BSA) conjugated to a near-infrared quencher dye to track of lymphatic drainage from the eye. ${ }^{17}$ BSA and HSA have a similar amino acid composition and are nearly identical with the exception that HSA has one tryptophan group where BSA has two, and HSA also has a higher content of valine. ${ }^{104}$

The presence of various groups and regions on BSA and HSA enable dual labeling where one signaling compound can be conjugated to the protein via the amine-reactive groups, where another can use the hydrophobic region as a binding site. Since BODIPY is hydrophobic, it can be used as a signaling compound by placing it within the hydrophobic regions of the protein via an entropic effect. Another dye like QC-1, CF770, or IRDye 800CW can be used as the second signaling compound using an amine-ester covalent bond. Additionally, thiol-reactive BODIPY can be used to label to proteins as well. ${ }^{105}$

By utilizing the targeting properties of serum albumin, dyes can be labelled to the protein to alter and potentially improve the imaging properties of the tracer. Therefore, a hybrid tracer using serum albumin as a targeting ligand will enable live tracking of $\mathrm{AH}$ outflow to the lymphatic system as well as fluorescence in situ and microscopic imaging validation which would be useful for testing novel treatments for glaucoma.

\subsection{Thesis hypothesis, specific aims, and organization}

\subsubsection{Hypothesis}

Labeling a quencher dye and a fluorescent dye to the same protein will create a hybrid tracer that may be suitable for both in vivo photoacoustic imaging, and post-mortem fluorescence imaging (for validation of in vivo photoacoustic imaging results).

\subsubsection{Specific aims}

The specific aims of this thesis are: 
SA1: To determine a standardized method to characterize photoacoustic contrast agents for lymphatic imaging.

SA2: To characterize several near-infrared tracers for photoacoustic imaging

SA3: To select an optimal near-infrared dye for in vivo photoacoustic imaging of lymphatic drainage

SA4: To combine the selected near-infrared dye and a fluorescent dye for dual labeling of bovine serum albumin to image lymphatic drainage of $\mathrm{AH}$ from the eye to lymph node

SA5: To characterize the combined tracer using the same methods determined by SA1

SA6: To test the combined photoacoustic and fluorescent, lymph node targeting contrast agent, by injecting it into the anterior chamber of mice and imaging the mice with photoacoustic and fluorescent imaging, in vivo and post-mortem respectively.

\subsubsection{Thesis Organization}

This thesis is organized in six chapters. Chapter 1 covers the introduction to glaucoma, its connection to lymphatics and lymphatic imaging. Chapter 1 also introduces the basic concepts of photoacoustic imaging and contrast agents, and the use of serum albumin as a targeting ligand for the lymphatic system. Chapter 2 is the basis of a manuscript submitted to the Molecular Imaging and Biology Journal that describes in depth methods to characterize photoacoustic contrast agents for lymphatic imaging. Chapter 3 is a second manuscript that builds upon the characterization techniques described in Chapter 2 to aid in the development of a hybrid photoacoustic and fluorescent contrast agent. It is deemed ready for submission by the supervisors. Thesis discussion and conclusions are presented in Chapters 4, and Chapter 5 presents future directions and preliminary in vivo data using the hybrid contrast agent described in Chapter 3, and some conclusions based on preliminary data. Chapter 6 contains all of the references. 


\section{Chapter 2 : An Approach to Test the Suitability of a Commercial Near- Infrared Dye for Quantitative In Vivo Photoacoustic Lymphatic Imaging}

This chapter presents a manuscript that was submitted to the Journal of Molecular Imaging and Biology

Submission \#: MIBI-D-19-00220

Submission date: $23 / 07 / 2019$

An Approach to Test the Suitability of a Commerical Near-Infrared Dye for Quantitative In Vivo Photoacoustic Lymphatic Imaging

Kirsten Cardinell ${ }^{1-3}$, Neeru Gupta ${ }^{1,2,4,7,8}$, J Carl Kumaradas ${ }^{3}$, Xun Zhou ${ }^{1,2}$, Hyacinth Irving ${ }^{1}$, Paola Luciani $^{9,10}$, and Yeni H. Yücel ${ }^{1-6}$

${ }^{1}$ Keenan Research Centre for Biomedical Science, Li Ka Shing Knowledge Institute, St. Michael’s Hospital, Toronto, Ontario, Canada

${ }^{2}$ Department of Ophthalmology and Vision Sciences, St. Michael's Hospital, University of Toronto, Toronto, Ontario, Canada

${ }^{3}$ Department of Physics, Faculty of Science, Ryerson University, Toronto, Ontario, Canada

${ }^{4}$ Department of Laboratory Medicine \& Pathobiology, St. Michael's Hospital, University of Toronto, Toronto, Ontario, Canada

${ }^{5}$ Institute of Biomedical Engineering, Science and Technology (iBEST), St. Michael's Hospital, Ryerson University, Toronto, Ontario, Canada

${ }^{6}$ Department of Mechanical Engineering, Faculty of Engineering and Architectural Science, Ryerson University, Toronto, Ontario, Canada 
${ }^{7}$ Glaucoma Unit, St. Michael's Hospital, Toronto, Ontario, Canada

${ }^{8}$ Dalla Lana School of Public Health, University of Toronto, Toronto, Ontario, Canada

${ }^{9}$ Department of Pharmaceutical Technology, Institute of Pharmacy, Friedrich Schiller University Jena, Otto Schott Strasse 41, 07745 Jena, Germany

${ }^{10}$ Pharmaceutical Technology Group, Department of Chemistry and Biochemistry, University of Bern, Freiestrasse 3, 3027 Bern, Switzerland

\section{Preface}

This paper, entitled "An Approach to Test the Suitability of a Commercial Near-Infrared Dye for Quantitative In Vivo Photoacoustic Lymphatic Imaging", describes a study where we determined and implemented a method of non-biased characterization of photoacoustic contrast agents. I was the primary researcher of this study. I designed and executed the experiments, performed the data analysis, and prepared the figures.

I formulated the research concept with Dr. Yeni Yucel and Dr. Neeru Gupta, both of whom provided feedback and suggestions for experimental design and contributed intellectual ideas to this work. The statistical analysis outlined in section 2.3.5.5 was performed by Hyacinth Irving. Dr. Paola Luciani provided insights into the tested dyes. Xun Zhou provided insights on experimental design and results as well as provided me with the appropriate training. Dr. Carl Kumaradas provided insights on experimental results through committee meetings. All authors took part in editing this manuscript.

\subsection{Abstract}

There is a lack of an objective standardized approach to compare and select contrast agents for photoacoustic imaging. To determine the optimal contrast agent for photoacoustic imaging of lymphatic drainage, three commercially available near-infrared dyes were investigated: dark quencher dye, IRDye® 
QC-1, and fluorescent dyes IRDye ${ }^{\circledR} 800 \mathrm{CW}$ and $\mathrm{CF}^{\mathrm{TM}} 770$. The characteristics assessed in vitro included minimum detectable concentration (MDC), linear range, and photostability. All three dyes demonstrated similar MDCs of 0.89 (QC-1), 1.99 (IRDye 800CW), and 2.81 $\mu \mathrm{M}$ (CF770). Linear range was largest for CF770, followed by IRDye 800CW with two distinct ranges. QC-1 showed the narrowest linear range. However, QC-1 was more sensitive to variation in concentration with a slope an order of magnitude steeper than the other dyes. QC-1 was the most photostable followed by CF770. This work provides a systematic unbiased approach to compare dyes to optimize in vivo quantitative photoacoustic experiments.

Keywords: Contrast Agent, Minimum Detectable Concentration, Limit of Detection, Linear Range, Photobleaching

\subsection{Introduction}

Recent developments in the field of photoacoustic (PA) imaging include, but are not limited to, the advancements of super-resolution PA imaging, ${ }^{106}$ functional PA microscopy,${ }^{107-110}$ and PA tomography. ${ }^{111}$ The development of contrast agents ${ }^{65,112-118}$ has also been important for PA imaging. However, standardized methods to compare various contrast agents and to select them for specific applications in PA imaging are rather limited. ${ }^{67}$

The acoustic detection in PA imaging is based on the optical absorption of two categories of chromophores: endogenous and exogenous. Endogenous chromophores such as oxygenated and deoxygenated hemoglobin, or melanin are constitutively present in the blood and skin, respectively. Currently, endogenous chromophores are used in a limited subset of clinical ailments. ${ }^{65}$ Alternatively, exogenous chromophores are not present in the body and are often introduced in PA imaging as contrast agents to increase the contrast between the signal and background, increase the maximum imaging depth, locate biological entities, or to observe biological functions in vivo. Contrast agents consist of two primary components: a targeting ligand, and a signaling compound. The targeting ligand is used to specify the location of the contrast agent in vivo and it is selected based on the biological entity or process of interest. 
The selection of the targeting ligand depends on numerous parameters and is beyond the scope of this paper. A signaling compound is the source of the PA signal and can be categorized into three broad classes: metallic or semiconducting nanostructure, organic polymer, or near-infrared (NIR) dye. ${ }^{66,67}$

Of the three classes of signaling compounds, NIR dyes tend to have good tissue distribution and penetration, low toxicity, and fast clearance rates. ${ }^{66}$ Furthermore, NIR dyes can be conjugated to various proteins and enzymes $^{74}$ to obtain NIR tracers that can be used to track biodistribution and biological processes. We have performed this in previous work by tracking lymphatic drainage using labelled albumin. ${ }^{17}$ PA dyes should absorb in the NIR range within the "biological window" which is the range of wavelengths (from $700 \mathrm{~nm}$ to $900 \mathrm{~nm}$ ) where the absorption due to the endogenous chromophores oxygenated and deoxygenated hemoglobin, and water are at a minimum. ${ }^{119}$ To our knowledge, tests for quantitative standardization and characterization to compare new and current NIR dye signaling compounds for PA imaging are not yet well-established.

There are several important characteristics when selecting a PA signaling compound and these parameters will vary depending on the application. The background signal due to the presence of endogenous chromophores in the target tissue or compartment is important to consider in the selection of a tracer. Imaging of the lymph, whose PA background signal is extremely low owing to an aqueous fluid without endogenous chromophores, presents unique opportunities for the development of PA contrast agents.

In this study, we aim to develop a method to characterize and to help compare contrast agents in a lymphlike aqueous solution with minimum background signal. One pivotal characteristic of the contrast agents to be determined is the minimum detectable concentration (MDC), also known as limit of detection (LOD) ${ }^{120}$, defined as the lowest concentration at which the PA signal from the signaling compound can be distinguished from the background or control signal. It is desirable to have a smaller LOD for applications in which identifying the location of the signaling compound in low concentrations is important. 
Furthermore, to quantify relative changes in signaling compound detection in PA images, it is essential that the concentrations in the region of interest remain within the linear range of the PA signal vs concentration for the contrast agent. Therefore, the larger the linear range, the larger the range of quantification. The other relevant parameter is the slope within the linear range, the steeper the slope, the greater the sensitivity of the photoacoustic imaging to differentiate between concentrations of the contrast agent. Directly connected to the linear range is the limit of quantification (LOQ), defined as the minimum quantifiable concentration which can be equal to, or greater than, the LOD. Finally, photostability of the NIR dyes under continuous light exposure is an important characteristic to assess for quantitative photoacoustic imaging. The decrease in PA signal as a function of laser irradiation time and energy is an informative parameter to be assessed for each NIR dye. We will refer to this phenomenon as PA photobleaching. Some studies have already investigated the effects of laser power and energy on the PA photobleaching of various contrast agents. ${ }^{113-}$ ${ }^{115}$ An ideal signaling compound for experiments requiring repetitive PA imaging would be photostable, and would not undergo PA photobleaching, and would generate a consistent PA signal, regardless of light exposure during the imaging session. The lack of PA photobleaching would enable accurate quantification of the PA signal with multiple imaging sessions.

The NIR dyes suitable for PA could be either fluorescent or non-fluorescent- also known as dark quencherdyes. Fluorescent dyes enable the validation of PA images through gold standard fluorescence imaging modalities. However, since some of the energy absorbed by fluorescent dyes is converted into lower energy photons, less energy is converted into heat and in turn, a smaller PA signal is produced than an equivalent dye that doesn't fluoresce. Dark quencher dyes absorb the photons from fluorescent dyes (quench) and do not convert absorbed energy into photons which could potentially generate a stronger PA signal. ${ }^{121,122}$ Furthermore, we hypothesize that the lack of fluorescence from dark quencher dyes will result in less PA photobleaching. ${ }^{123}$

In this study, we compare three different commercial NIR dyes: IRDye 800CW, CF770, and IRDye QC-1. IRDye $800 \mathrm{CW}$ is a fluorescent dye that has already been used in PA imaging ${ }^{82,118,124-126}$ and is commonly 
used in various other applications like some cited here. ${ }^{79}, 80,127,128$ CF770, a remarkably photostable fluorophore that has been used for in vivo fluorescence imaging ${ }^{129,130}$ and western blot experiments, ${ }^{131}$ has not yet been used in PA imaging to the best of our knowledge.

IRDye QC-1 is a broad range NIR dark quencher that we used as the PA signaling compound in our aforementioned study. ${ }^{17}$ It has also been used in other PA studies as a quencher for IRDye $800 \mathrm{CW} .{ }^{78,121}$ QC-1 has also been compared against other NIR dyes in terms of photoacoustic performance and detection when formulated in biocompatible nanoemulsions. ${ }^{65}$

In this study, we propose a comprehensive in vitro characterization method for signaling compounds for the use of quantitative in vivo PA imaging. Several commercially available NIR dyes were compared with a PA imaging by determining the minimum detection concentration, the relationship between the dye concentration and the PA signal, and the effects of PA photobleaching on PA signal.

\subsection{Materials and Methods}

\subsubsection{Dye Selection}

Commercially available NIR dyes were selected based on two primary optical absorption properties: peak absorption wavelength, and molar extinction coefficient. In biological optical imaging, there is a NIR window between $700 \mathrm{~nm}$ and $900 \mathrm{~nm}$ where the absorption of the endogenous chromophores is at a minimum allowing for maximum depth penetration. ${ }^{119}$ It is important for any optical imaging signaling compound to have a peak absorbance in this window to have valid in vivo imaging use. All NIR dyes were selected to have their peak absorption wavelength within this range. Furthermore, the molar extinction coefficient is a value that is directly related to the light absorbance of a material which is one of the main parameters that dictate the magnitude of the generated PA pressure wave. ${ }^{132}$ Therefore, a higher molar extinction coefficient is likely to produce a higher PA signal which will also increase the signal to noise ratio. 


\subsubsection{Dye Preparation}

Several NIR dyes were selected for testing as PA contrast agents: QC-1 (IRDye QC-1; LI-COR Biosciences, NE, USA), CF770 (Biotium, CA, USA), and IRDye 800CW (IRDye 800CW LI-COR, NE, USA). QC-1 is a dark quencher dye, and CF770 and IRDye 800CW are NIR fluorescent dyes with maximum emission wavelengths at $797 \mathrm{~nm}$, and $789 \mathrm{~nm}$ in PBS, respectively (Hyclone Laboratories Inc, UT, USA).

Table 1. The list of NIR dyes used in this study along with their maximum absorption wavelength $\left(\lambda_{\max }\right.$ abs $)$, molar extinction coefficient $(\varepsilon)$, cost per $0.5 \mathrm{mg}$, and supplier. ${ }^{.}$

\begin{tabular}{lcclc}
\hline Dye & $\lambda_{\max a b s}$ & $\varepsilon$ & Cost Per 0.5 mg (USD) & Supplier \\
\hline IRDye QC-1 & $737 \mathrm{~nm}$ & $96,000 \mathrm{M}^{-1} \mathrm{~cm}^{-1}$ & $\$ 130$ & LI-COR \\
CF770 & $770 \mathrm{~nm}$ & $220,000 \mathrm{M}^{-1} \mathrm{~cm}^{-1}$ & $\$ 41$ & Biotium \\
IRDye 800CW & $774 \mathrm{~nm}$ & $240,000 \mathrm{M}^{-1} \mathrm{~cm}^{-1}$ & $\$ 130$ & LI-COR \\
\hline
\end{tabular}

Each dye was delivered in lyophilized powder form and prior to experiments, a stock solution was made by mixing $250 \mu \mathrm{L}$ of ultra-pure water (Wisent bioproducts, QC, Canada) with the lyophilized powder in the original packaging as instructed by the manufacturer. The concentration of the stock solution was calculated using the molar mass of the dye, and the mass of the powder provided by the manufacturer.

\subsubsection{Absorbance Readings}

Absorbance spectrum measurements were taken with the Biotek plate reader spectrophotometer (MultiMode Reader Synergy Neo2 BioTek, VT, USA) after further diluting each dye in PBS to approximately 25 $\mu \mathrm{M}$. Absorbance was measured from duplicate $2 \mu \mathrm{L}$ samples of each dye concentration scanned from 680

\footnotetext{
${ }^{1}$ Prices obtained from suppliers as of February 2019. Prices were converted into USD for 0.5 mg of product. Amount of product does not necessarily reflect what is available for sale by the supplier.
} 
$\mathrm{nm}$ to $980 \mathrm{~nm}$ in $1 \mathrm{~nm}$ steps with PBS as a control. The concentration of each sample was determined by Beer-Lambert's law with the molar extinction coefficient at the peak absorbance wavelength provided by the manufacturer.

\subsubsection{Phantom Preparation}

A cylindrical phantom mold was made from two modified $20 \mathrm{~mL}$ syringes (diameter of $1.85 \mathrm{~cm}$, Becton, Dickinson and Company, NJ, USA) and two capillary tubes (Inner diameter of $1.1 \mathrm{~mm}$ and outer diameter of $1.5 \mathrm{~mm}$, Thermo Fisher Scientific, ON, Canada) to create nearly identical phantoms for each in vitro dye characterization experiment. The capillary tubes were placed symmetrically into holes on both sides of the cylindrical mold. A diagram of the phantom mold is shown in Figure 2-1.

Phantoms were made using a modified protocol from Deán-Ben et al. ${ }^{133}$ to provide a uniform light distribution to the dye samples. Agar powder (75 mg, Sigma-Aldrich, MO, USA) was mixed with $50 \mathrm{~mL}$ of deionized water and brought to a boil in a microwave oven (Sanyo Electric, Osaka, Japan). The agar mixture was allowed to cool for $5 \mathrm{~min}$ at room temperature prior to adding $2.5 \mathrm{~mL}$ of warmed Intralipid ${ }^{\circledR}$ (20\%, Sigma-Aldrich, MO, USA) to make the final mixture $1 \%$ Intralipid and $1.5 \%$ agar. The agarintralipid mixture was then poured into the phantom mold and allowed to solidify at room temperature. Once solidified, the phantom was removed from the mold to be used for PA imaging.

\subsubsection{Relationship between PA Signal and Dye Concentration}

\subsubsection{Dye Preparation}

From the stock solutions, 17 concentrations were made for each dye $(200,175,150,125,100,75,50,40$, $25,20,10,5,2.5,1.25,0.625,0.313$, and $0.156 \mu \mathrm{M})$ by diluting in PBS assuming that the stock concentration is accurate. Prior to PA imaging, the absorbance of duplicate $2 \mu \mathrm{L}$ samples of each dye concentration was scanned with PBS as a control using the spectrophotometer from $680 \mathrm{~nm}$ to $980 \mathrm{~nm}$ in 1

$\mathrm{nm}$ steps. The average maximum absorbance for each dye sample concentration was used to calculate the 
actual concentration of the dye sample which varied significantly from the planned concentrations either due to inaccurate dye powder masses or inexact quantities of added ultrapure water to the stock solution.

\subsubsection{PA Imaging}

Capillary tubes were cut to approximately $5 \mathrm{~cm}$ segments. For each concentration, approximately $15 \mu \mathrm{L}$ was placed inside each capillary tube. Both ends of the tube were sealed with a few millimeters of white polymerclay (Sculpey, Michael's Craft Store, TX, USA) where super glue (Elmer's Krazy glue, Elmers' Products, NC, USA) was used to enhance the seal. Each dye concentration was imaged inside the agarintralipid phantom next to a capillary tube of PBS as control. Dyes were imaged using the MSOT inVision 128 (iThera Medical, Heidelberg, Germany). Prior to imaging, the MOST was turned on and allowed to warm up for 40 min to allow the water to reach $32{ }^{\circ} \mathrm{C}$, the generic preset temperature for the MSOT imager.

The phantom was oriented inside the MSOT imaging chamber using the MSOT phantom holder with the sample and control horizontal to one another and the phantom was centered in the imaging chamber. Each dye concentration sample was imaged from $680 \mathrm{~nm}$ to $980 \mathrm{~nm}$ in $5 \mathrm{~nm}$ steps every $2 \mathrm{~mm}$ along $1 \mathrm{~cm}$ of the phantom. At each position, 3 frames were taken and averaged for each wavelength. Each concentration for each dye was imaged 3 times ( 3 different samples in their own respective capillary tubes) to demonstrate reproducibility and observe any variation. 

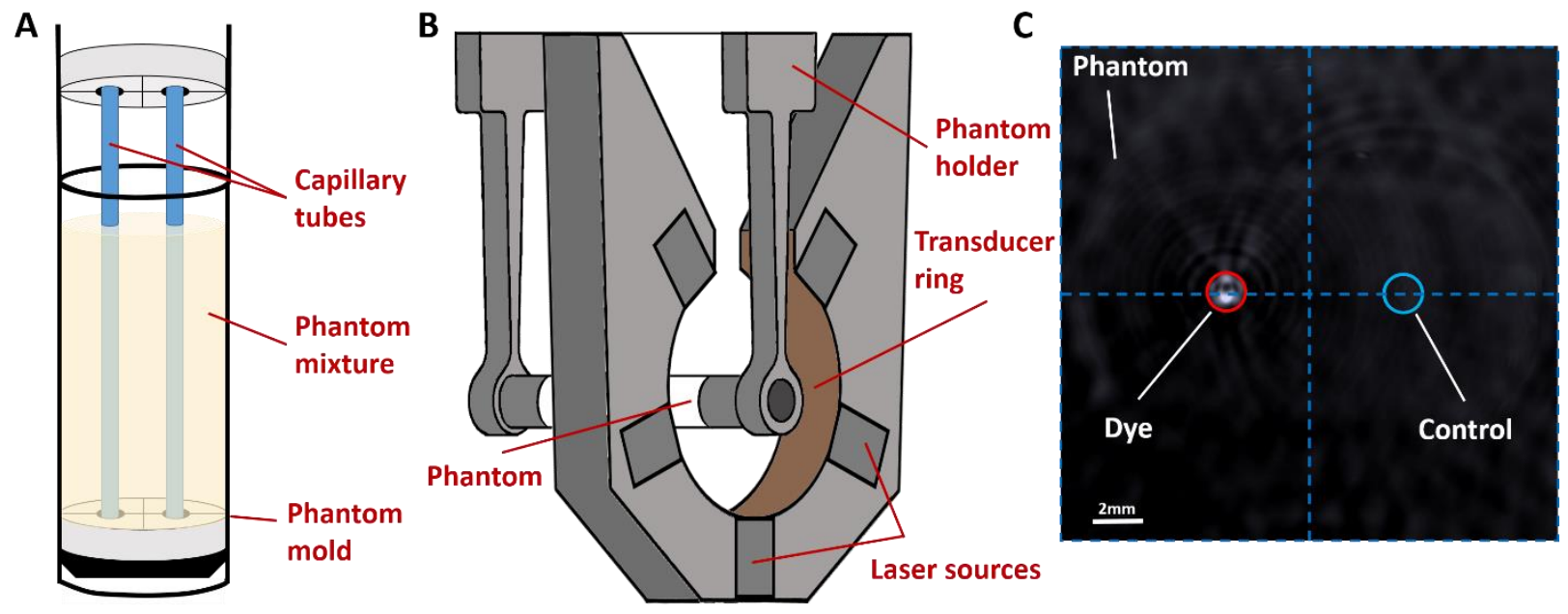

Figure 2-1. Phantom preparation and imaging set up. A) Diagram of a home-made phantom. B) Phantom holder and PA multispectral tomography animal imaging system (MSOT) diagram. C) Reconstructed PA image of the dye imaging set up. The red circle shows the dye region of interest, and the blue circle shows the control (PBS) region of interest. The intersect of dotted lines indicate the center of the transducer ring shown in B. The scale bar indicates $2 \mathrm{~mm}$. Diagrams A) and B) are approximated and not to scale.

\subsubsection{PA Image Data Analysis}

PA images were reconstructed using the Backprojection algorithm within the native viewMSOT software. To obtain the PA spectra of each dye to compare to the molar extinction coefficients, circular regions of interest (ROIs) were taken surrounding the dye and control (PBS without dye) capillary tubes with an area of $1.75 \mathrm{~mm}^{2}$. The mean pixel intensity (MPI) within the ROIs was recorded for each wavelength and the MPIs for the dye ROIs were plotted as a function of wavelength and are shown in Figure 2-2. The PA spectra were taken from the dyes with concentrations near $25 \mu \mathrm{M}$.

To determine the relationship between concentration and PA detection, spectral unmixing was done using wavelengths from $700 \mathrm{~nm}-900 \mathrm{~nm}$ with the water molar extinction coefficient spectrum provided by the viewMSOT software taken from data in the literature as well as the molar extinction coefficients of the imaged dyes as calculated by the spectrophotometer readings mentioned in section 2.3.1. The linear 
regression spectral unmixing algorithm was used to determine the unit-less relative proportions of water and the dye.

The same sized circular ROIs were made and the MPIs of the detected dye for both the dye ROI and the control ROI were recorded for the entire capillary. PA image slices were visually observed for any slices that contained the capillary with the polymer clay end seals or completely missing the capillary tube due to imperfect alignment. Data obtained from these slices were removed from the data set. The remaining MPIs for each concentration and sample were averaged and are shown in Figure 2-3(vide infra) for all dyes.

\subsubsection{Minimum Detectable Concentration}

The LOD in vitro was calculated using EP17, Protocols for Determination of Limits of Detection and Limits of Quantitation described by Armbruster and Pry. ${ }^{120}$ The limit of blank (LOB, $L_{\text {blank }}$ ) was calculated using (1), which was then used to calculate the LOD ( $\left.L_{\text {detection }}\right)$ using (2).

$$
\begin{gathered}
L_{\text {blank }}=\mu_{\text {blank }}+1.645\left(\delta_{\text {blank }}\right) \\
L_{\text {detection }}=L_{\text {blank }}+1.645\left(\delta_{\text {low conc.sample }}\right)
\end{gathered}
$$

Linear regression lines of best fit were made for the MPIs as a function of concentration for each dye using data from 5 concentrations starting from one concentration below the LOB moving to higher concentrations (approx. 0 to approx. $25 \mu \mathrm{M}$ ). The MDC for each dye was estimated using the linear regression line of best fit equation and the LOD. Standard deviation was calculated from the line of best fit. MDCs and standard deviations are shown in 
Table 2.

\subsubsection{Linear Range}

The linear range of each dye was determined through the statistical methods described in EP6-A. ${ }^{134}$ Linear mixed effects models for small sample inference were used to estimate the parameters, outlier rejection, and recalculation of the fitted regression model. In all models, dye identity was included as a random factor to account for repeated measurements. The mean pixel intensities (MPIs) of the dyes, dependent on the dye concentration, were initially fit with models with a cubic function for the entire range of dye concentrations. If the coefficients of the cubic terms were significantly different from zero $(\mathrm{P}<0.05)$, then the plot of the MPI as a function of concentration was observed to remove data points from either the high concentration end or the low concentration end. The data was continuously fitted to the cubic function until the cubic coefficients became non-significant. Subsequently, a quadratic function was fitted to the data and if the coefficients of the quadratic term were significantly different from zero, then the same steps of removing data were taken as mentioned previously until the quadratic term was non-significant. Finally, the linear model was estimated and the various parameters including the y-intercept, slope, residual, and respective variances between samples were derived. The final estimated linearity model was assessed for lack of fit by basic evaluation of a plot of MPI and derived concentration levels. All analysis were completed using Stata 15.1 (StataCorp 2017).

\subsubsection{PA Photobleaching: PA Signal versus Imaging Time}

\subsubsection{Dye Preparation}

A single concentration for each dye was made from their respective stock solutions by diluting in PBS. Concentrations for each dye were selected such that the mean pixel intensities were similar for all of the dyes. (2.3.4). Prior to PA imaging, the absorbance of duplicate $2 \mu \mathrm{L}$ samples of each dye was scanned with PBS as a control using the spectrometer from $680 \mathrm{~nm}$ to $980 \mathrm{~nm}$ in $1 \mathrm{~nm}$ steps. As described above, the 
maximum absorbance for each dye and concentration were used to calculate the actual concentration of the dye. After PA imaging, the imaged dye samples were read again with the spectrophotometer under the same settings.

\subsubsection{PA Imaging}

The setup for the PA photobleaching experiment was nearly identical to that described in 2.3.5.2. However, for these experiments, the capillary tubes were carefully measured and cut to $3.5 \mathrm{~cm}$ segments. So that for each concentration, approximately $6 \mu \mathrm{L}$ was placed inside each capillary tube. For these experiments, each dye sample was imaged at their respective maximum peak absorbance every $0.5 \mathrm{~mm}$ along the length of the capillary tube. At each position, 3 frames were taken and averaged. The capillary tube was imaged repetitively, without delay for 1 hour as done in another study. ${ }^{114}$ Additionally, 1 hour of imaging is comparable to the total cumulative imaging time of mice in our quantitative study. ${ }^{17}$ As described above, each dye was imaged 3 times ( 3 different samples in their own respective capillary tubes) to demonstrate reproducibility and observe any variation.

\subsubsection{PA Image Data Analysis}

PA images were reconstructed using the Backprojection algorithm. Circular regions of interest were taken surrounding the dye and control capillary tubes with an area of $1.75 \mathrm{~mm}^{2}$ and the MPIs were recorded. A sample of 10 image slices per scan was selected for data analysis. The average and standard deviation MPI of the 10 slices for each of the three imaging samples were taken for each time point. The average MPI was then normalized to the maximum MPI of each dye respectively. The normalized MPI as a function of time is shown in Figure 2-6 (vide infra).

Using MATLAB codes provided by iThera Medical, the average laser energy at each time point was extracted from the data and the average summation of energy over time was noted for all three dyes. 


\subsection{Results}

The PA spectra of the dyes showed a change in the spectral shape compared to the molar extinction coefficient spectra. Most notably, the MPI was much higher at the shorter wavelengths $(<700 \mathrm{~nm})$ compared to the molar coefficient spectra. Furthermore, the peak absorption wavelength was characterized by a 5-10 nm hypsochromic shift for all dyes in the PA spectra.
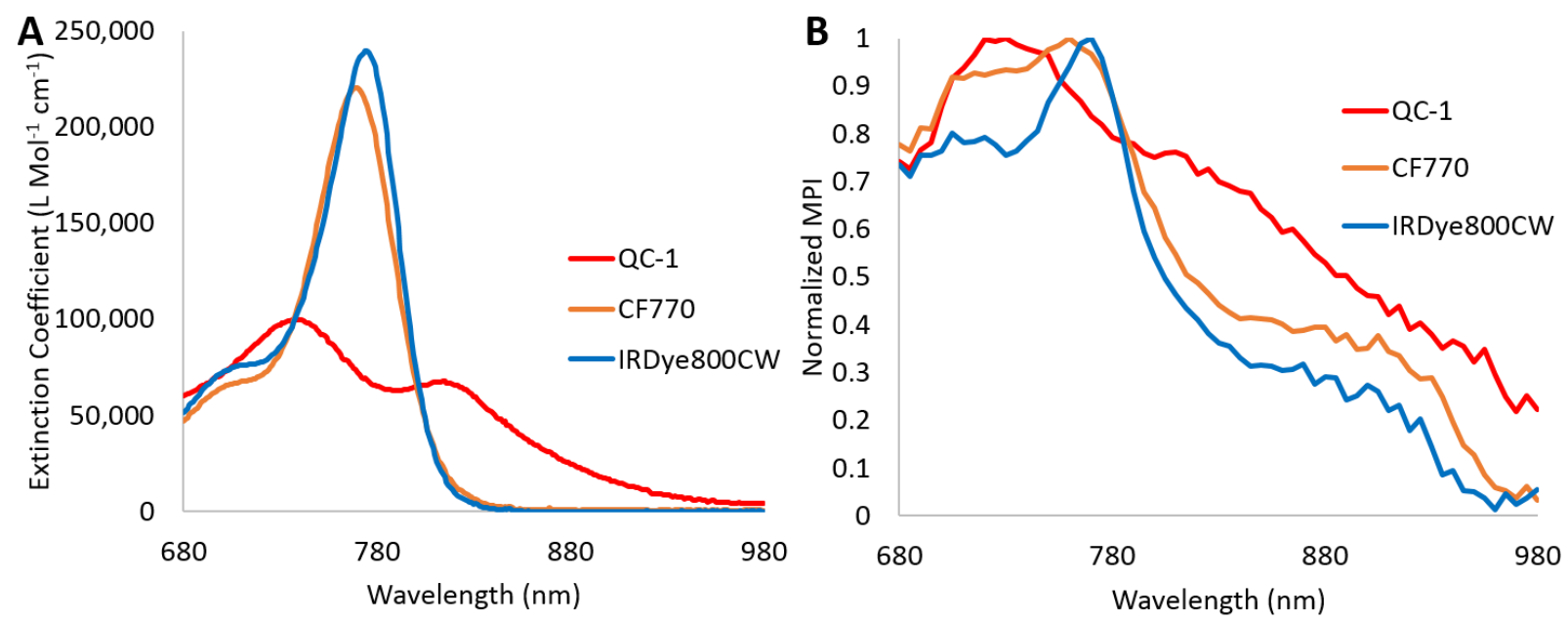

Figure 2-2. Near-infrared dye molar extinction coefficients and absorption spectra measured from 680 to $980 \mathrm{~nm}$. A) Molar extinction coefficients determined from the spectrophotometer measurements. B) PA mean pixel intensity (MPI)

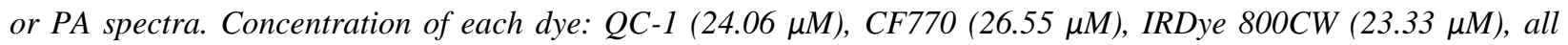
diluted in PBS.

\subsubsection{Relationship between PA Signal and Dye Concentration}

The PA signals, or MPIs, for each dye in Figure 2-3 show that at concentrations higher than approximately $10 \mu \mathrm{M}$, MPIs for QC-1 are remarkably larger than the other NIR dyes such as CF770, and IRDye 800CW that have similar MPIs. Further analysis determining the minimum detectable concentration (MDC) for each dye indicates that IRDye 800CW has the lowest MDC, followed by CF770 and QC-1 (Figure 2-4 and 
Table 2).
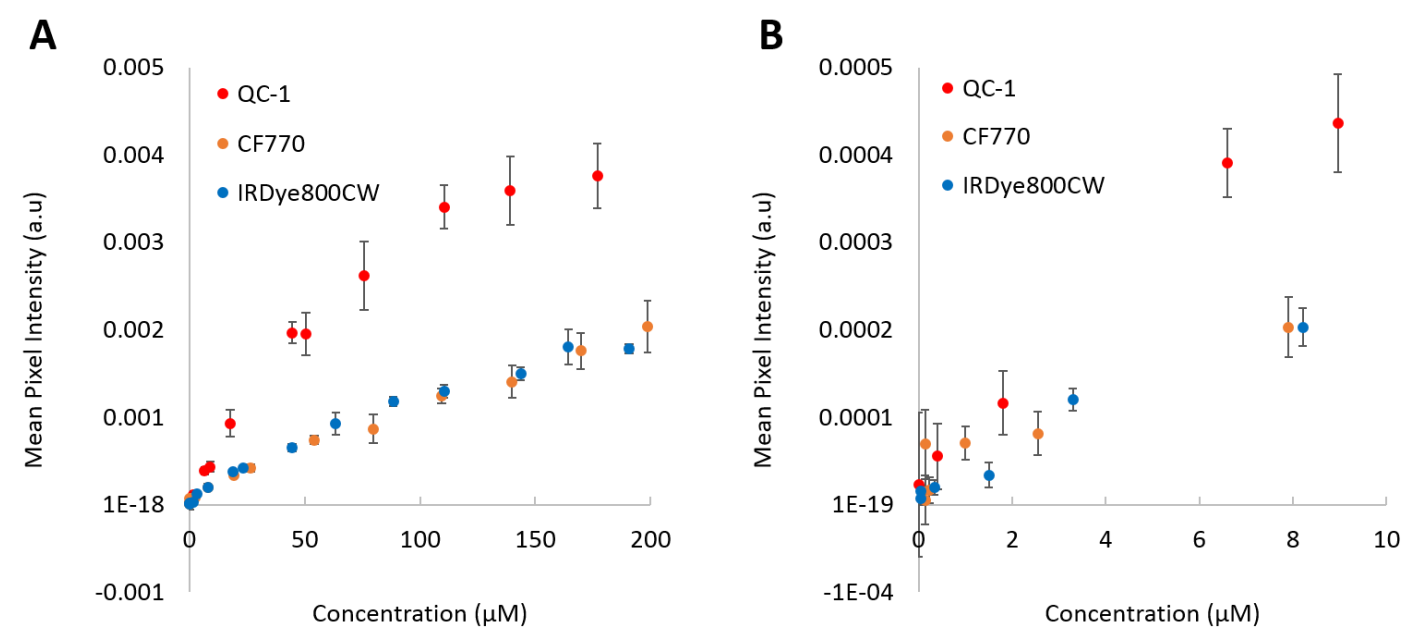

Figure 2-3. PA signal (mean pixel intensity, MPI) as a function of dye concentration for all near-infrared dyes. A) Concentration ranges from 0 to approximately $200 \mu \mathrm{M}$. B) Concentration ranges from 0 to approximately $10 \mu M$. Error bars represent the standard deviations of MPI between the imaging slices in the three samples.

\subsubsection{Minimum Detectable Concentration (MDC)}

IRDye QC-1 was shown to have the smallest MDC followed by IRDye $800 \mathrm{CW}$ and CF770 respectively. All MDCs were determined to be relatively close with small standard deviations. 

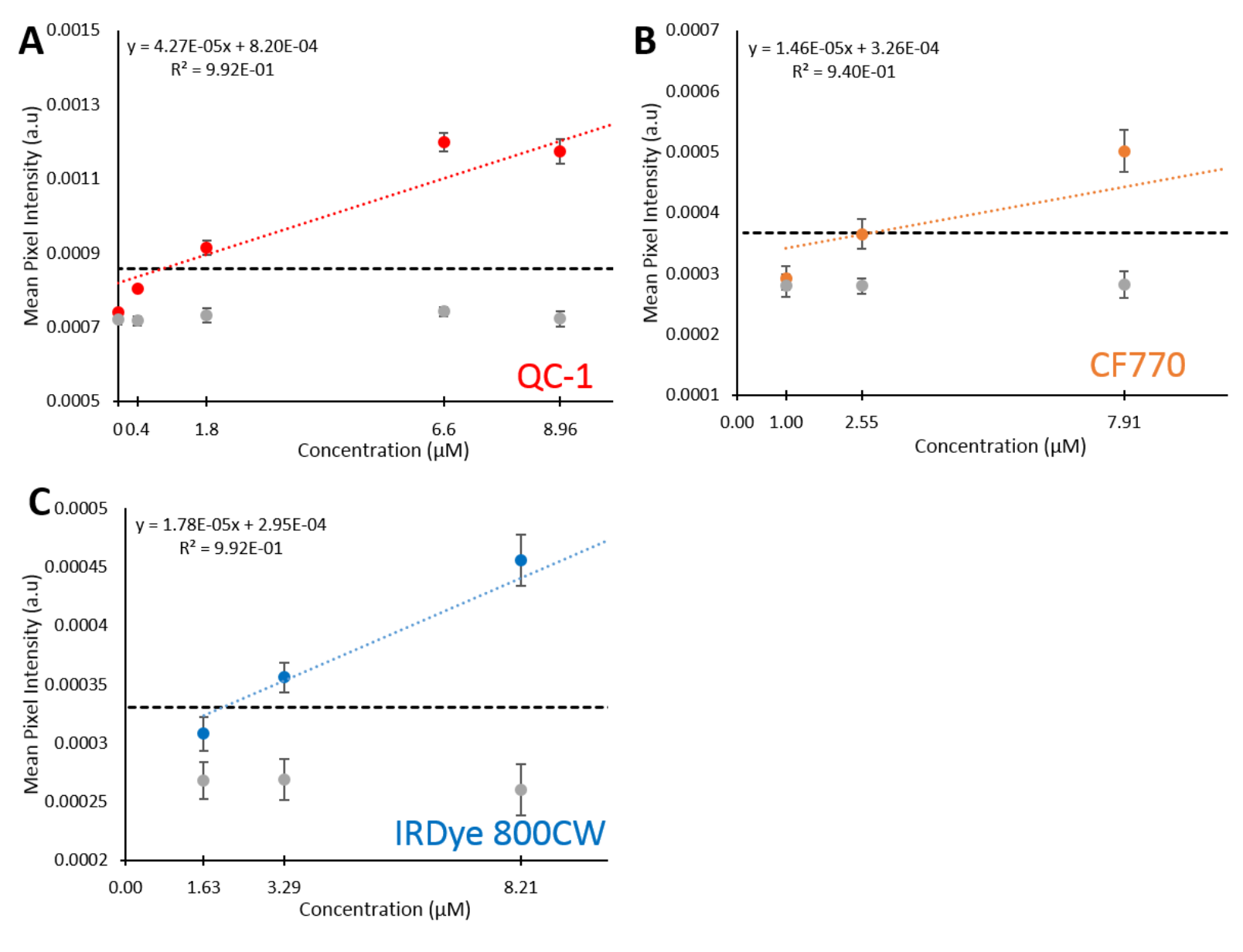

Figure 2-4. Minimum Detectable Concentration and PA signal (mean pixel intensity, MPI) as a function of dye concentration. Concentration ranges from $0 \mu \mathrm{M}$ to approximately $25 \mu \mathrm{M}$. A) IRDye QC-1 B) CF770 C) IRDye 800CW. Limit of Detection line shown in black. MDC for each dye is: QC-1 (0.000992 a.u.), CF770 (0.000367 a.u.), and IRDye $800 C W$ (0.00033 a.u.). PBS MPI is shown in gray. Error bars represent the standard deviations of MPI between the imaging slices in the three samples. 
Table 2. Minimum detectable concentration: The estimated minimum concentration detectable in vitro by PA imaging for NIR dyes. ${ }^{2}$

\begin{tabular}{ll}
\hline Dye & Minimum Detectable Conc. \\
\hline QC-1 & $0.89 \mu \mathrm{M} \pm 1.28 \mu \mathrm{M}$ \\
CF770 & $2.81 \mu \mathrm{M} \pm 2.89 \mu \mathrm{M}$ \\
IRDye 800CW & $1.99 \mu \mathrm{M} \pm 0.78 \mu \mathrm{M}$ \\
\hline
\end{tabular}

\subsubsection{Linear Range}

Graphical representation of the linear ranges for each dye is shown in Figure 2-5. The fluorescent CF770 dye has the largest statistically linear range of concentrations compared to the other two dyes. Two separate linear ranges were detected for IRDye $800 \mathrm{CW}$. The second, larger range for IRDye $800 \mathrm{CW}$ extended to the end of the concentration range tested, implying that the range may extend past the maximum quantifiable concentration indicated. However, after performing an additional experiment using concentrations from 87 to $306 \mu \mathrm{M}$, we found that there is a statistically linear range of IRDye $800 \mathrm{CW}$ from 63.5 to $306 \mu \mathrm{M}$ and a significant variance between the slopes of the three samples when these additional concentrations are added. QC-1 had the smallest linear range. The QC-1 showed the steepest linear range slope compared to CF770 and IRDye 800CWas indicated in Table 3.

\footnotetext{
${ }^{2}$ Standard deviation values were calculated using the expected concentration values from the line of best fit equations.
} 
Table 3. Linear range: The approximate ranges between two concentrations where the photoacoustic signal intensity is linearly related to the concentration.

\begin{tabular}{lll}
\hline Dye & Linear Range & Slope of Linear Range \\
\hline QC-1 & 0 to 75.7 & $3.563 \mathrm{E}-5$ \\
CF770 & 19.5 to 198.9 & $9.27 \mathrm{E}-6$ \\
IRDye 800CW & 0 to $23.3 \& 44.5$ to 190.6 & $1.82 \mathrm{E}-5 \& 7.00 \mathrm{E}-6$ \\
\hline
\end{tabular}

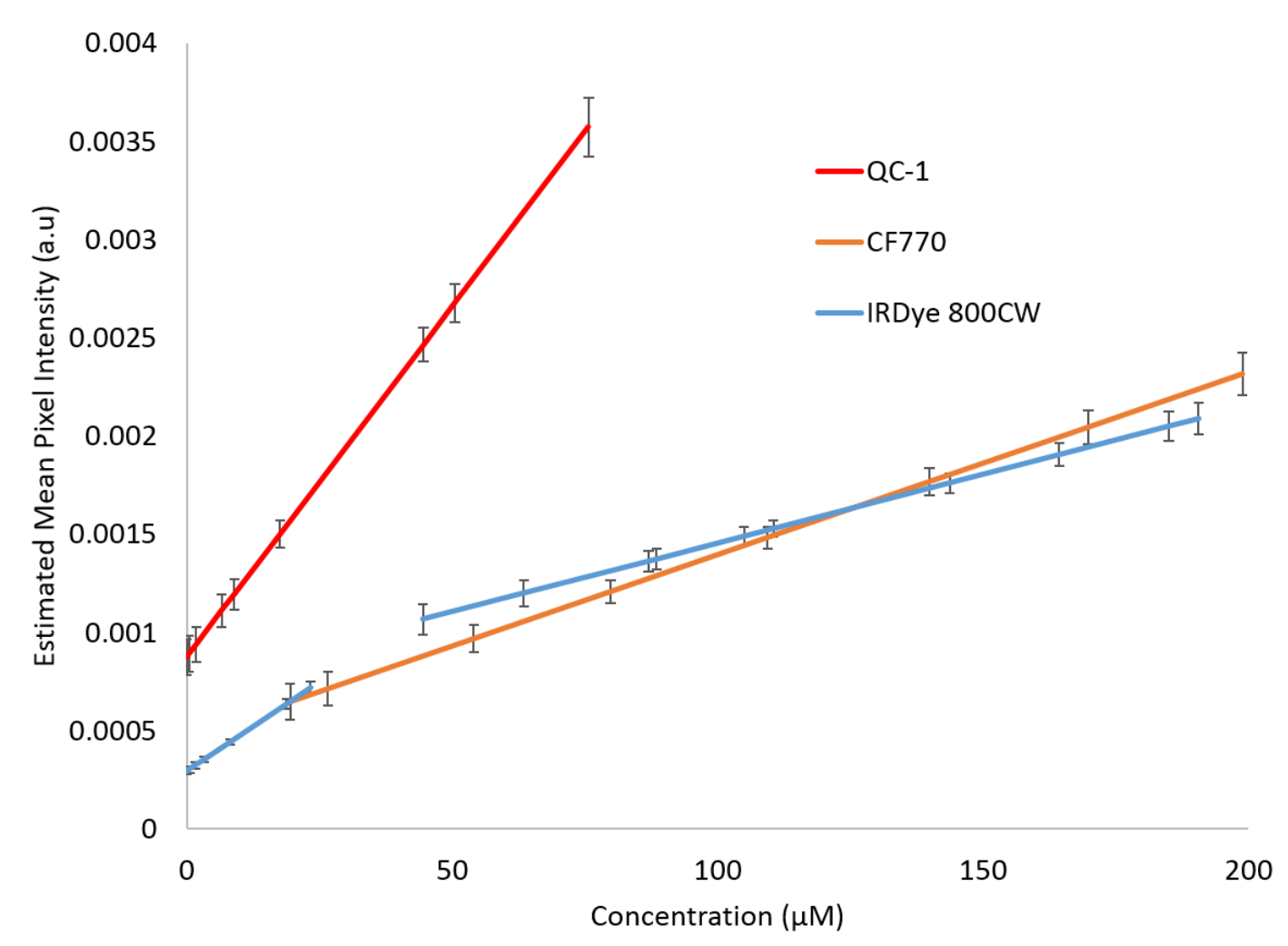

Figure 2-5. The line of best fit for the statistically linear range of concentrations for each dye. Bars represent the 95\% confidence interval. 


\subsubsection{PA Photobleaching: PA Signal versus Imaging Time}

The PA signal, or MPI, of the three NIR dyes showed a significant decrease after 60 min of imaging. QC1 showed the least amount of PA photobleaching having a decrease in signal of approximately $11 \%$. CF770 follows QC-1 with a decrease of $26 \%$, and IRDye $800 \mathrm{CW}$ has a decrease of $40 \%$. The average laser energy values integrated over time for the QC-1, CF770, and IRDye 800CW samples are 5.673, 5.877, and 5.709 $\mathrm{J} \cdot \mathrm{s}$ respectively with the largest difference in energies being between the QC-1 and CF770 samples. CF770 had a 3.6\% higher average summation of energy over time. We speculate that the steeper decline in MPI observed over time for CF770 compared to QC-1 is not entirely due to the slightly higher energy the CF770 samples were exposed to.

It is noteworthy that the effect of PA photobleaching had on the QC-1 samples do not appear to take effect until after 10 min of imaging. A similar observation can be made within 5 min of imaging for CF770.

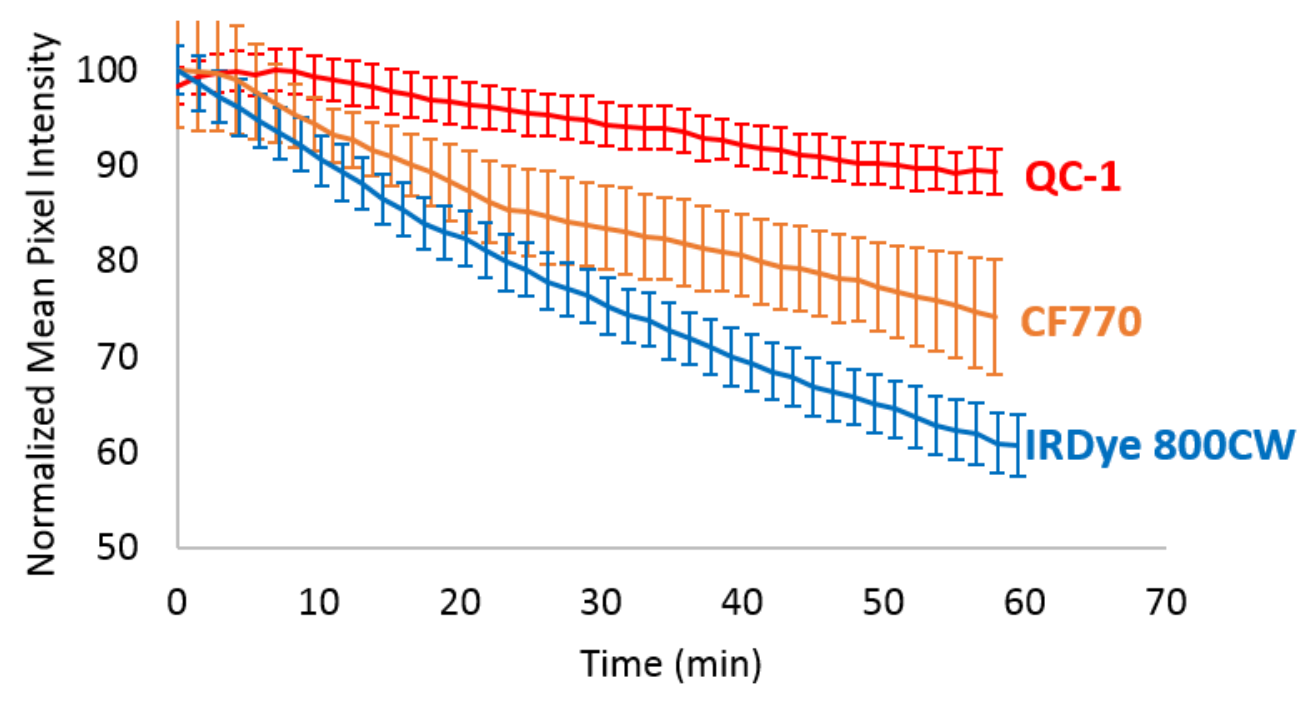

Figure 2-6. The normalized PA signal (mean pixel intensity, MPI) as a function of imaging time (total 1 hour) for QC1 (red), CF770 (orange), and IRDye 800CW (blue). Error bars represent the standard deviation between the three imaging sessions of three separate dye samples. 


\subsection{Discussion}

The results of these characterization tests indicate that no single dye from the ones we tested is a superior signaling compound for all 3 criteria. QC-1 has the smallest MDC making it the most sensitive NIR chromophore among the ones tested. The relationship between PA signal and concentration is also linear at these low concentrations for QC-1 meaning that the MDC is also equivalent to the minimum quantifiable concentration. However, at concentrations above approximately $80 \mu \mathrm{M}$, the relationship between the PA signal and concentration is not linear, making it a much narrower range of quantifiable concentrations by PA imaging when compared to CF770 and IRDye $800 \mathrm{CW}$. However, within the linear range, the slope is steeper for QC-1 compared to the other two dyes. QC-1 with the steepest slope, the photoacoustic imaging is more sensitive to detect variation in concentration compared to CF770 and IRDye 800CW. Furthermore, QC-1 showed the least amount of PA photobleaching in this study. Based on these results, QC-1 is a good choice of signaling compound for studies that require high PA sensitivity and/or repetitive PA imaging; however, since the linear range is so small, accurate quantification of QC-1 dye at higher concentrations is not feasible without the use of fluence correction methods. ${ }^{135}$

CF770 had the highest MDC of the three dyes tested; however, the MDC was still comparable to the other two dyes and should not be eliminated as a PA signaling compound for that reason alone. For the range of concentrations tested within this study, CF770 had the largest measured linear range. In terms of PA photobleaching, CF770 showed less photobleaching than IRDye $800 \mathrm{CW}$ and more than QC-1. If PA imaging applications require fluorescence imaging for validation, CF770 would be a good choice for a signaling compound provided that PA imaging is not performed for long periods of time (over approximately $5 \mathrm{~min}$ ) to reduce the effects of PA photobleaching for more accurate quantification. However, one could potentially quantify photobleached data, by taking into account the PA photobleaching 
rate and the total amount of light exposed to the signaling compound during the imaging sessions. The latter, which potentially involve complex fluence calculations, was beyond the scope of this paper.

Lastly, IRDye $800 \mathrm{CW}$ showed a small MDC as well as two distinct linear ranges. The presence of two linear ranges alone adds complexity to quantification; however, since there is a significant effect of PA photobleaching that occurs with IRDye $800 \mathrm{CW}$, it makes the dye difficult to quantify when repeated measurements over time are required. Despite PA photobleaching being a disadvantage in terms of PA quantification, it may have other potential uses such as light dose monitoring during near-infrared lightbased therapies. ${ }^{136}$

These experiments characterized commercially available NIR dyes as signaling compounds for PA imaging. Researchers choosing between NIR dyes, or other signaling compounds, for various applications should alter the assay described above to better suit their parameters; however, all parameters should be the same for all the dyes being tested.

FDA approved indocyanine green (ICG) was another NIR dye that was initially considered for this study due to clinical use; however, due to its well-established instability ${ }^{84}$, and poor water solubility, it was not included in this investigation.

In this study, three dyes were compared in vitro in aqueous medium mimicking lymph embedded within a light scattering phantom. The systematic approach described in this study may be also useful when studying contrast agents in more complex physiologically relevant media such as blood.

\subsection{Conclusion}

In conclusion, we developed a quantitative method for characterizing and comparing near-infrared dyes as signaling compounds for photoacoustic contrast agents. The criteria for comparison include the range of concentrations for which the relationship between concentration and photoacoustic signal is statistically linear, the minimum detectable concentration, and the effect repetitive imaging has on photoacoustic signal. 
These criteria are a way to measure the quantifiable range, sensitivity, and photostability respectively for a photoacoustic system and provide scientists with the insights on which signaling compound to use for their experimental needs. 


\section{Chapter 3 : A Hybrid Tracer for Photoacoustic and Fluorescence Imaging of Lymphatic Drainage from the Eye}

This chapter presents a manuscript that is deemed ready for submission by supervisors and co-authors Dr. Yeni Yucel and Dr. Carl Kumaradas.

Authors:

Kirsten Cardinell ${ }^{1-3}$, Neeru Gupta ${ }^{1,2,4,8,9}$, Bryan Koivisto ${ }^{7}$, J Carl Kumaradas ${ }^{3}$, Xun Zhou ${ }^{1,2}$, and Yeni H. Yücel $^{1-6}$

Affiliations:

${ }^{1}$ Keenan Research Centre for Biomedical Science, Li Ka Shing Knowledge Institute, St. Michael’s Hospital, Toronto, Ontario, Canada

${ }^{2}$ Department of Ophthalmology and Vision Sciences, St. Michael's Hospital, University of Toronto, Toronto, Ontario, Canada

${ }^{3}$ Department of Physics, Faculty of Science, Ryerson University, Toronto, Ontario, Canada

${ }^{4}$ Department of Laboratory Medicine \& Pathobiology, St. Michael's Hospital, University of Toronto, Toronto, Ontario, Canada

${ }^{5}$ Institute of Biomedical Engineering, Science and Technology (iBEST), St. Michael's Hospital, Ryerson University, Toronto, Ontario, Canada

${ }^{6}$ Department of Mechanical Engineering, Faculty of Engineering and Architectural Science, Ryerson University, Toronto, Ontario, Canada

${ }^{7}$ Department of Chemistry and Biology, Faculty of Science, Ryerson University, Toronto, Ontario, Canada 
Corresponding author: Yeni H. Yücel

Keenan Research Centre for Biomedical Science, St. Michael's Hospital

30 Bond Street

209 LKSKI Room 409

Toronto Ontario M5B 1W8

Phone: $416864-6060$ ext:77594

E-mail: yucely@smh.ca

\section{Preface}

Having previously demonstrated a method to characterize photoacoustic contrast agents using non-biased methods described in Chapter 2, we wanted to use this method of characterization to aid in the development of a hybrid photoacoustic-fluorescent contrast agent for use in lymphatic imaging. This paper, entitled "A Hybrid Tracer for Photoacoustic and Fluorescence Imaging of Lymphatic Drainage from the Eye", describes a study where we developed a hybrid photoacoustic-fluorescent contrast agent through labeling two different dyes to the same protein. I was the primary researcher of this study. I designed and executed the experiments, performed the data analysis, and prepared the figures.

I formulated the research concept with Dr. Yeni Yucel and Dr. Neeru Gupta, both of whom provided feedback and suggestions for experimental design and contributed intellectual ideas to this work. Dr. Bryan Koivisto provided the BODIPY dye used in this study and provided insights on the chemistry of the dye and the methods for labeling the BODIPY to the protein. Xun Zhou provided insights on experimental design and results and provided appropriate training. Dr. Carl Kumaradas provided insights on experimental results through committee meetings. All authors took part in editing this manuscript. 


\subsection{Abstract}

Photoacoustic (PA) imaging for biomedical applications often requires contrast agents to improve signal to background ratios. In vivo PA images are generated by computer-assisted qualitative and quantitative data analysis. Therefore, adding another imaging modality such as fluorescence imaging can validate in vivo findings. The availability of a single contrast agent that can be effectively used for in vivo PA and postmortem fluorescence imaging is currently lacking and would be advantageous in the field. Here we developed a hybrid tracer with the purpose of in vivo PA imaging (PAI) of lymphatics and post-mortem fluorescence imaging validation. Bovine serum albumin (BSA) was dually labeled with dark near-infrared (NIR) quencher dye QC-1 for PAI and Pentamethyl BODIPY for fluorescence imaging. The QC-1-BSABODIPY (QBB) tracer was characterized using a commercial PAI system (680 to $980 \mathrm{~nm})$ to determine the minimum detectable concentration (MDC), linear, or quantifiable, range, and the photostability. The MDC, linear range and photostability of the QBB tracer was $1.22 \mu \mathrm{M}$, from 0 to $73 \mu \mathrm{M}$, and a $14 \%$ decrease in PA signal after 1-hour laser light exposure, respectively. Furthermore, QBB remained fluorescent after repetitive laser exposure. The development of a hybrid tracer is promising for the PAI and requires stronger binding of the BODIPY to BSA to further improve the fluorescence imaging.

Keywords: Contrast Agent, Minimum Detectable Concentration, Limit of Detection, Linear Range, Photobleaching

\subsection{Introduction}

Photoacoustic (PA) imaging is a three-dimensional (3D) hybrid imaging modality that combines optical and acoustic imaging. ${ }^{137}$ The use of near-infrared (NIR) laser wavelengths within the range of 700 to 950 nm, known as the first NIR optical window, ${ }^{138}$ allows the imaging of deeper tissues since the absorption of oxygenated and deoxygenated hemoglobin are at a minimum. The generation of PA images require computer-assisted qualitative and quantitative analysis of the PA data ${ }^{64,139}$ and these software-generated images often require post-mortem or biopsy validation in biology and pathology applications. ${ }^{140-145}$ Post- 
mortem validation is also required to confirm both qualitative and quantitative results of PAI since this is a relatively new imaging modality. Histological validation of PAI is often performed by fluorescence imaging. ${ }^{146}$ In our previous work, we assessed in vivo lymphatic drainage from the eye in mice using PAI and a NIR non-fluorescent contrast agent. ${ }^{17}$ To validate in vivo PA images we performed, in a separate group of animals, post-mortem fluorescence imaging after the injection of a NIR fluorescent tracer. Development of a hybrid photoacoustic-fluorescent contrast agent would enable in vivo photoacoustic imaging and post-mortem fluorescence validation in the same animals, reduce interindividual variation, and improve the correlation studies between in vivo PA and fluorescence images.

As PAI depends on the absorption of NIR light by molecules, the detection of tissues in the body that do not absorb light in this range (ie. the non-pigmented tumors, lymph, and cerebrospinal fluid) requires the use of exogenous contrast agents. A contrast agent consists of two components: a targeting ligand and a signaling compound. ${ }^{51}$ A targeting ligand is used to target a specific biological entity or process. For example, albumin is drained from interstitial tissue by lymphatics and is accumulated in the lymph nodes, ${ }^{75}$ and is therefore often used as a targeting ligand in lymphatic imaging. ${ }^{147-151}$ A signaling compound, for PAI, is the component of the tracer that generates the photoacoustic signal. There are several categories of signaling compound: metallic nanoparticles, organic nanostructures, and NIR dyes. Of the three categories, NIR dyes are the smallest and can be used to label ligands such as proteins and antibodies with a minimal change in the size or in biocompatibility of the protein or antibody. ${ }^{51}$

Several studies have demonstrated the use and applications of combined photoacoustic and fluorescence imaging. ${ }^{152-159}$ Indocyanine green (ICG) is the only FDA approved NIR dye and as such, has been widely used as a contrast agent for both photoacoustic and fluorescence imaging. ${ }^{157-159}$ However, due to the dyeinstability and spectral shape changes as a function of concentration ${ }^{84,160}$, due to aggregation ${ }^{161,162}$, the quantification of the ICG signal becomes complicated. Alterations of ICG $^{163}$ or changes in imaging parameters are required to reduce photobleaching. Other fluorescent dyes that have been used as contrast agents for dual photoacoustic and fluorescence imaging include cyanine dyes ${ }^{153,}{ }^{155}$ and fluorescein ${ }^{154}$. 
IRDye800CW, another NIR fluorescent dye, has been used for photoacoustic and fluorescence imaging for pancreatic cancer detection, ${ }^{164}$ and lymphatic imaging in mice. ${ }^{165}$ Using NIR fluorescent dyes enables the use of both fluorescence and PA imaging without the need for additional signaling compounds. However, a study performed by Laufer et al. demonstrated that non-fluorescent proteins, when compared to fluorescent proteins, have a higher photoacoustic generation efficiency and a significantly higher photostability. ${ }^{166}$ For PAI, a higher photostability is characterized by a stable PA signal after long-duration laser exposure. In this paper, we will refer to a decrease in PA signal as a function of laser exposure as photoacoustic photobleaching. It is therefore likely that a non-fluorescent dye would exhibit similar photostability properties to a non-fluorescent protein resulting in high photoacoustic signal generation and less photoacoustic photobleaching. In addition, due to the peak absorbance wavelength of NIR fluorescent dyes being within the PAI wavelength range, the high energy of the photoacoustic laser may induce a photochemical change in the dye which results in the dye having reduced or completely inhibited fluorescence, a.k.a photobleaching. Therefore, a NIR non-fluorescent dye would be advantageous for PAI. An example of a NIR non-fluorescent dye is IRDye QC-1 (LI-COR), developed as a broad-range dark quencher for Förster resonance energy transfer assays. ${ }^{76}$ A quencher dye is able to absorb, or quench, the photons emitted by fluorescent dyes. The most efficient quenching occurs when there is a significant overlap of the absorption spectrum of the quencher dye and the fluorescent spectrum of the donor fluorescent dye and the dyes are in close proximity. ${ }^{76}$ Additionally, QC-1 has also been previously used as a photoacoustic contrast agent. ${ }^{17,}$ 65, 67, 78, 167 In our previous study, QC-1 demonstrated desirable photoacoustic imaging properties including a linear, or quantifiable, range with a steep slope allowing for easier differentiation between close concentrations, a low minimum detectable concentration, and good photostability with minimal change in photoacoustic signal generation with increasing imaging time. ${ }^{168}$ All these properties are desirable for quantitative photoacoustic imaging that requires multiple imaging sessions. 
However, a non-fluorescent dye like QC-1 cannot be detected using the fluorescence imaging modalities and therefore cannot be used to validate the PA images. A method to maintain the PA signal stability as well as to obtain fluorescence signal is to use a contrast agent that contains both a non-fluorescent and fluorescent dye. A fluorescent dye that requires higher energy photons to excite, and therefore shorter peak absorption and fluorescence wavelengths $(<700 \mathrm{~nm})$, would prevent the dye from being photobleached by the PA laser during imaging with the commercial photoacoustic tomography (MSOT, wavelength range $(680 \mathrm{~nm}-980 \mathrm{~nm})$, iThera Medical Inc.) used in this study. This fluorescent dye would therefore not produce a photoacoustic signal and can be paired with the dark NIR quencher QC-1 so long as the fluorescence of the dye is outside the quenching range of QC-1 (520 to $809 \mathrm{~nm}) .{ }^{76}$ Additionally, a dye that has maximum absorbance at a wavelength shorter than the NIR wavelength range of the MSOT photoacoustic imaging system would likely not undergo fluorescence photobleaching that would occur from the repetitive photoacoustic laser irradiation.

There are several well known dyes that have peak fluorescence wavelengths outside both the quenching range of QC-1 and the MSOT imaging range including, but not limited to, fluorescein, various Alexa Fluor dyes, and BODIPY derivatives (Table 4).

Table 4. Properties of commonly use fluorescent dyes with peak emission wavelengths below the imaging range of the $M S O T$ and the quenching range of $Q C-1$.

\begin{tabular}{lllll}
\hline Fluorophore & $\varepsilon$ & Abs max $(\mathrm{nm})$ & Emission max $(\mathrm{nm})$ & Quantum yield \\
\hline Alexa Fluor 405 & 35,000 & 401 & 421 & - \\
Alexa Fluor 350 & 19,000 & 346 & 442 & - \\
Alexa Fluor 488 & 73,000 & 495 & 519 & 0.92 \\
Pentamethyl BODIPY & 93,000 & 493 & 503 & 0.85 \\
Fluorescein & 76,900 & 491 & 512 & 0.93 \\
\hline
\end{tabular}


Fluorescein has a large molar extinction coefficient and a high quantum yield. However, it also has several limitations including having relatively high photobleaching, $\mathrm{pH}$-sensitive fluorescence, and quenching tendencies of their own fluorescence when conjugated to biopolymers. ${ }^{169}$

A suggested alternative to fluorescein is Alexa Fluor 488. Of the Alexa Fluor dyes with fluorescence peaks outside the quenching range of QC-1, Alexa Fluor has the largest molar extinction coefficient. Alexa Fluor 488 also has a similar absorption and emission spectra to fluorescein without any of the same limitations. However, Alexa Fluor dyes are highly charged which can result in the perturbation of conjugate functional properties. $^{90}$

4,4-Difluoro-4-bora-3a, 4a-diaza-s-indacenes (BODIPYs) (Figure 3-1. General structure of BODIPY : Z,Y $=\beta$ or 2,6 positions, $X=$ meso position, $\mathrm{W}=\alpha$ or 3,5 positions, $V=1,7$ positions or other $\beta$ positions) is a non-polar, electrically neutral family of dyes that has been used in a variety of biological imaging and advanced material applications due to their highly desirable properties such as high absorption coefficients, and sharp emission peaks exhibiting high quantum yields and therefore, they produce strong fluorescence signals. BODIPY can be used for intracellular imaging labeling reagents, ${ }^{170,}{ }^{171}$ biologically-relevant sensors, ${ }^{172}$ and photodynamic therapy ${ }^{86}$ In addition, they are stable under physiological conditions making them suitable for the fluorescence labeling of proteins for in vivo studies. ${ }^{86}$

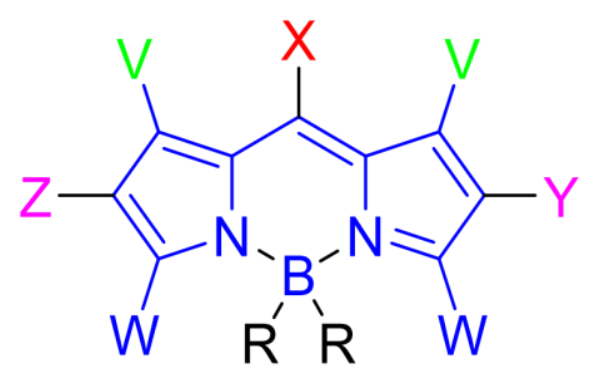

Figure 3-1. General structure of BODIPY : Z,Y $Y=\beta$ or 2,6 positions, $X=$ meso position, $W=\alpha$ or 3,5 positions, $V=$ 1,7 positions or other $\beta$ positions. Pentamethyl BODIPY is $Z, Y=-H ; X, V, W=$ methyl. 
Specifically, the BODIPY derivative used in this study was the pentamethyl BODIPY (BODIPY 493/503) derivative whose synthesis has been previously reported. ${ }^{173}$ While their spectroscopic characteristics can be adjusted by rational design, BODIPYs are often insoluble in aqueous environments without significant chemical modification. As a result of this insolubility, optimal performance in bioimaging is not possible. However, in this work, we report the physical encapsulation of this pentamethyl BODIPY within Bovine serum albumin (BSA).

We hypothesize that by combining the pentamethyl BODIPY dye that fluoresces outside the quenching range of QC-1 alongside the QC-1 dye, a hybrid photoacoustic-fluorescent contrast agent could be developed. To combine the QC-1 and BODIPY dyes into a hybrid contrast agent specifically for the purpose of lymphatic imaging, ${ }^{75}$ both dyes can be labeled to serum albumin. Serum albumin is the most common protein in blood with a concentration of 3.5-5.0 g/dl. It is $66 \mathrm{kDa}$ in size with dimensions of 40 by 40 by $140 \mathrm{~A}^{\circ} .{ }^{91}$ Serum albumin has previously been labeled with indocyanine green (ICG) for photoacoustic tumor imaging. ${ }^{100}$ Furthermore, serum albumin has a high uptake in the lymphatic system ${ }^{75}$ and we used bovine serum albumin (BSA) in our previous work as a targeting ligand for the lymph nodes. ${ }^{17}$ Prior to using the hybrid contrast agent in vivo, it needs to be tested in vitro for use in PA and fluorescence imaging. We previously described a non-biased, systematic method for characterizing dyes for PA imaging based on three criteria: minimum detectable concentration (MDC), linear range, and photostability. ${ }^{168}$ The MDC is the lowest concentration at which a dye can be detected with a photoacoustic imaging device. The linear range is the range of concentrations for which the relationship between the photoacoustic signal, and the concentration of the dye is linear. The photostability is the measurement of how consistent the photoacoustic signal is over repetitive laser exposure, and, if the dye is fluorescent, the fluorescence signal after repetitive laser exposure. Both linear range and photostability are important for quantitative in vivo PAI where the change in concentration of a tracer is important. 
In this study, we designed a hybrid tracer for in vivo photoacoustic tomography and post-mortem fluorescence imaging. We characterized this hybrid tracer for its photoacoustic and fluorescence imaging properties in vitro.

\subsection{Materials and Methods}

\subsubsection{Dual Labeling to Albumin}

IRDye QC-1 (NHS Ester, LI-COR Biosciences, NE, USA) was conjugated to bovine serum albumin (BSA; Sigma-Aldrich, MO, USA; MW: $66.4 \mathrm{kDa}^{174}$ ) to make a QC-1/BSA solution using the protocol described by the manufacturers (IRDye800CW Protein Labeling Kit-High MW, LI-COR Biosciences, NE, USA). After conjugation, the sample was filtered with a $3 \mathrm{kDa}$ Amicon Ultra-15 Centrifugal Filter Unit (SigmaAldrich, MO, USA) to remove any free dye. The filtered QC-1/BSA absorbance was measured from 250 $\mathrm{nm}$ to $980 \mathrm{~nm}$ in $1 \mathrm{~nm}$ steps with a microplate spectrophotometer (Synergy Neo2, BioTek, VT, USA) to determine the dye to protein ratio using the equation below:

$$
\frac{D}{P}=\frac{\left[\frac{A_{\text {max }}}{\varepsilon_{\text {Dye }}}\right]}{\left(\frac{A_{280}-C F * A_{\max }}{\varepsilon_{\text {protein }}}\right)}
$$

Where $A_{\max }$ is the absorbance of the QC-1 labeled protein at QC-1's maximum absorbance wavelength $(737 \mathrm{~nm}), A_{280}$ is the absorbance of the conjugated protein at the maximum absorbance wavelength of the

protein $(280 \mathrm{~nm}), \varepsilon_{\text {Dye }}$ is the extinction coefficient of the QC-1, $\varepsilon_{\text {protein }}$ is the extinction coefficient of the protein, and $\mathrm{CF}$ is the correction factor calculated by the following equation:

$$
C F=\left(\frac{A_{280}}{A_{\max }}\right)_{\text {dye }}
$$


Where $A_{280}$ and $A_{\max }$ here are the absorbance at $280 \mathrm{~nm}$ (the maximum absorbance wavelength of the BSA protein) and the maximum absorbance respectively for the free QC-1 dye.

Pentamethyl BODIPY is a strongly fluorescent BODIPY dye that is more hydrophobic than other BODIPYs, and the methylated periphery makes it less susceptible to degradation. Owing to the need to label the BODIPY into a hydrophobic BSA cavity through entropic labeling, it was selected as the most robust fluorophore, with the lowest propensity for leakage. The BODIPY was dissolved in N, NDimethylformamide (DMF) to make a stock solution of $25 \mathrm{mM}$. The BODIPY solution was mixed with the QC-1/BSA solution at a 10:1 BODIPY to BSA ratio. The QC-1/BSA/BODIPY (QBB) mixture was incubated at $37{ }^{\circ} \mathrm{C}$ for 5 hours at 40 RPM. After incubation, the solution was filtered with a new centrifugal filter unit to remove any free BODIPY dye and the absorbance of the sample was read with the spectrophotometer from $250 \mathrm{~nm}$ to $980 \mathrm{~nm}$ in $1 \mathrm{~nm}$ steps and the fluorescent endpoint was measured using the same device with a $490 \mathrm{~nm}$ excitation and a $520 \mathrm{~nm}$ emission.

To estimate the dye to protein ratio for the BODIPY in this dual-dye tracer, two additional correction factors were calculated:

$$
C F_{1}=\left(\frac{A_{750}}{A_{\max }}\right)_{\text {BODIPY }}
$$

Where $A_{750}$ and $A_{\max }$ here are the absorbance at $750 \mathrm{~nm}$ (the maximum absorbance of the QC-1 dye bound to BSA) and the maximum absorbance respectively for the free BODIPY dye, and:

$$
C F_{2}=\left(\frac{A_{497}}{A_{\max }}\right)_{\mathrm{QC}-1}
$$

Where $A_{750}$ and $A_{\max }$ here are the absorbance at $497 \mathrm{~nm}$ (the maximum absorbance of the free BODIPY dye) and the maximum absorbance respectively for the QC-1 dye bound to BSA. 
With these two correction factors we can make two systems of equations:

$$
\begin{aligned}
& A_{750 \mathrm{~nm}}=A_{\mathrm{Qc}-1 \text { at } 750 \mathrm{~nm}}+C F_{1} * A_{\mathrm{BODIPY}} \text { at } 450 \mathrm{~nm} \\
& A_{450 \mathrm{~nm}}=A_{\mathrm{BODIPY}} \text { at } 450 \mathrm{~nm} \\
& A_{2} * A_{\mathrm{Qc}-1} \text { at } 750 \mathrm{~nm}
\end{aligned}
$$

Where the total absorbance values $\left(A_{750 \mathrm{~nm}}\right.$ and $\left.A_{450 \mathrm{~nm}}\right)$ are the spectrophotometer readings for absorbance at their stated wavelengths. The two unknown values are $A_{\mathrm{Qc}-1}$ at $750 \mathrm{~nm}$ and $A_{\mathrm{BODIPY}}$ at $450 \mathrm{~nm}$ where $A_{\mathrm{Qc}-1}$ at $750 \mathrm{~nm}$ is the absorbance at $750 \mathrm{~nm}$ contributed by QC-1 and $A_{\text {BODIPY at } 450 \mathrm{~nm}}$ is the absorbance at $450 \mathrm{~nm}$ contributed by BODIPY.

After calculating for the two unknown absorbance values, they can be used to calculate the concentration of each dye using the Beer-Lambert law:

$$
C=\frac{A}{L * \varepsilon}
$$

Where $\mathrm{C}$ is the dye concentration, $\mathrm{A}$ is the absorbance value calculated as described above, $\mathrm{L}$ is the pathlength and $\varepsilon$ is the molar extinction coefficient of the dye. Assuming the dye to protein ratio does not change for QC-1 when BODIPY is added, we can use the following equation to estimate the dye to protein ratio of BODIPY in the QBB solution:

$$
\left(\frac{D}{P}\right)_{\mathrm{BODIPY}}=\frac{C_{\mathrm{BODIPY}}}{C_{\mathrm{QC}-1}} *\left(\frac{D}{P}\right)_{\mathrm{QC}-1}
$$

Prior to the dual labeling, we tested the labeling of BODIPY to BSA alone by following the same procedure described above, however, the incubation time was only 3 hours. To increase the labeling efficiency for the dual label tracer, the incubation time was increased to 5 hours. The dye to BSA ratio we estimated based on these calculations is 2.29:1, and 1.90:1 for QC-1 and BODIPY, respectively within the QBB tracer. 


\subsubsection{Phantom Preparation}

Light scattering, non-absorbing phantoms were made as described previously. ${ }^{168}$ A cylindrical mold was made using two modified $20 \mathrm{~mL}$ syringes (Becton, Dickinson and Company, Franklin Lakes, NJ, USA). and two capillary tubes (Inner diameter of $1.1 \mathrm{~mm}$ and outer diameter of $1.5 \mathrm{~mm}$, Thermo Fisher Scientific, ON, Canada). The phantoms were made by boiling a mixture of agar powder ( $75 \mathrm{mg}$ Sigma-Aldrich, MO, USA) in $50 \mathrm{~mL}$ of deionized water in a microwave oven (Sanyo Electric, Osaka, Japan). After allowing for 5 minutes of cooling, $2.5 \mathrm{~mL}$ of warmed Intralipid ${ }^{\circledR}$ (20\%, Sigma-Aldrich, MO, USA) was added to the mixture and allowed to cool and solidify within the phantom mold at room temperature. The final mixture of the phantom was $1 \%$ intralipid and $1.5 \%$ agar. The phantom was removed from the mold for PAI.

\subsubsection{Dye Preparation}

The stock solution of QBB was diluted using PBS to 17 concentrations using the calculated QC-1 concentration $(200,175,150,125,100,75,50,40,25,20,10,5,2.5,1.25,0.625,0.313$, and $0.156 \mu \mathrm{M})$. Prior to PA imaging, the absorbance of duplicate $2 \mu \mathrm{L}$ samples of each concentration and PBS as a control were measured using the spectrophotometer from $680 \mathrm{~nm}$ to $980 \mathrm{~nm}$ in $1 \mathrm{~nm}$ steps for concentration validation. Fluorescence endpoint measurements were taken with a $490 \mathrm{~nm}$ excitation and a $520 \mathrm{~nm}$ emission.

To determine the minimum detectable concentration (MDC) as well as the linear range, approximately 15 $\mu \mathrm{L}$ of each dye concentration was placed inside the capillary tubes cut to approximately $5 \mathrm{~cm}$ long. Both ends of each capillary tubes were sealed with a few millimeters of white polymerclay (Sculpey, Michael's Craft Store, TX, USA) and superglue was used to enhance the seal. Triplicates were made for each of the 17 concentrations. 


\subsubsection{Photoacoustic Imaging}

The QBB filled capillary tubes were imaged with a Multispectral Optoacoustic Tomographic system (MSOT inVision 128; iThera Medical, Heidelberg, Germany). Prior to imaging, the MSOT was turned on and allowed to warm up for 40 min for the water to reach $32{ }^{\circ} \mathrm{C}$, the generic preset temperature for the MSOT imager. The phantom was oriented inside the MSOT imaging chamber using the MSOT phantom holder with the QBB sample and PBS control horizontal to one another and the phantom was centered in the imaging chamber.

The phantom was imaged using wavelengths from $680 \mathrm{~nm}$ to $980 \mathrm{~nm}$ in $5 \mathrm{~nm}$ steps and was scanned every $2 \mathrm{~mm}$ along $1 \mathrm{~cm}$ of the phantom. At each position, 3 frames were taken and averaged for each wavelength. This was done for all 3 samples for all 17 concentrations to determine the MDC and the linear range.

\subsubsection{Photoacoustic Image Data Analysis}

All PA images were reconstructed using the Backprojection algorithm within the native viewMSOT software.

\subsubsection{Relationship between Photoacoustic Signal and Concentration}

Linear regression spectral unmixing was performed using the native viewMSOT software with wavelengths from $700 \mathrm{~nm}-900 \mathrm{~nm}$. The known water molar extinction coefficient spectrum provided by the software taken from literature as well as the molar extinction coefficient spectrum of QBB calculated by the spectrophotometer readings were the spectra used for the unmixing method. The linear regression spectral unmixing algorithm was used to determine unit-less relative proportions of water and the dye.

Circular regions of interest (ROIs) with an area of $1.75 \mathrm{~mm}^{2}$ were made and the MPIs of the detected QBB for both the QBB capillary ROI and the control capillary ROI were recorded for the entire length of each capillary. PA image slices were visually observed for any slices that contained the capillary with the 
polymer clay end seals or completely missing the capillary tube, and the data obtained from these slices were excluded from further analysis.

\subsubsection{Minimum Detectable Concentration}

The MDC was determined using the method described previously ${ }^{168}$ which was derived from the EP17, Protocols for Determination of Limits of Detection and Limits of Quantitation from the Clinical Laboratory Standards Institute. ${ }^{120}$ Using this protocol, a limit of the blank and limit of detection were calculated for the QBB dye.

Linear regression lines of best fit were made for the MPIs as a function of concentration for each dye using data from 5 concentrations starting from one concentration below the calculated limit of the blank moving to higher concentrations (approx. 0 to approx. $22 \mu \mathrm{M}$ ). The MDC for QBB was estimated by solving the linear regression line of best fit equation using the calculated limit of detection.

\subsubsection{Linear Range}

The method to determine the linear range of QBB was derived through the statistical methods described in EP6-A, Evaluating Quantitative Measurement Linearity from the Clinical Laboratory Standards Institute. ${ }^{134}$ Linear mixed effects models for small sample inference were used to estimate the parameters, outlier rejection, and recalculation of the fitted regression model. In all models, dye identity was included as a random factor to account for repeated measurements. The mean pixel intensities (MPIs) of the QBB, dependent on concentration, were fit with a cubic function model for the entire range of concentrations. If the cubic and quadratic coefficients were significantly different from zero $(\mathrm{P}<0.05)$, then the plot of the MPI as a function of concentration was observed to remove data points from either the high or low concentration end until the cubic coefficients became non-significant. Subsequently, a quadratic function was fitted to the data and if the coefficients of the quadratic term were significantly different from zero, then the same steps of removing data were taken until the quadratic term was non-significant. Finally, this 
reduced data set is considered to be within the linear range and a linear model was estimated. The final estimated linearity model was assessed for lack of fit by basic evaluation of a plot of MPI and derived concentration levels. Statistical analyses were completed using Stata 15.1 (StataCorp 2017).

\subsubsection{Photoacoustic Signal vs Repetitive Imaging}

For these experiments, the dye was prepared as described above. To determine the effect of extended laser exposure on the photoacoustic signal, an extra 3 capillary tubes, this time cut to exactly $3.5 \mathrm{~cm}$, were filled with $25 \mu \mathrm{M}$ of QBB.

To determine the photobleaching effect of extended laser light exposure on the photoacoustic signal, the phantom was imaged every $0.5 \mathrm{~mm}$ along $3 \mathrm{~cm}$ of the phantom to encompass the entire capillary filled with the QBB sample. The phantom was imaged with a wavelength of $750 \mathrm{~nm}$ which is the maximum absorption wavelength of QC-1 when conjugated to BSA and 3 frames were taken at each imaging slice. After

completing a scan of the entire phantom, the scan would continuously repeat until an hour of imaging time was completed.

The fluorescence signal of the QBB tracer was measured before and after the repetitive photoacoustic imaging using a $480 \mathrm{~nm}$ absorption and $520 \mathrm{~nm}$ emission.

Circular regions of interest were taken surrounding the QBB and control capillary tubes with an area of $1.75 \mathrm{~mm}^{2}$ within the reconstructed PA images and the MPIs were recorded. A sample of 10 image slices per scan was selected for data analysis. The average and standard deviation MPI of the 10 slices for each of the three imaging samples were taken for each time point. The average MPI was then normalized to the maximum MPI. 


\subsection{Results}

The absorbance spectrum of the free QC-1 dye, QC-1 dye labeled to BSA, free BODIPY dye, BODIPY labeled to BSA, and the hybrid QBB tracer are shown in Figure 3-2. When labeled to the BSA, the QC-1 absorption peak shifts to longer wavelengths (red-shift to approx. $750 \mathrm{~nm}$ ). In contrast, the absorption peak of BODIPY labeled to BSA shifts towards the shorter wavelengths (blue shift to approx. $450 \mathrm{~nm}$ ). There is also a noticeable change in spectral shape for both dyes when labeled to BSA. This change in spectral characteristics of the BODIPY and QC-1 dyes were also present when in the combined QBB tracer where the absorbance of QBB at $750 \mathrm{~nm}$ of the is attributed to the QC-1 and the absorbance at approximated 450 $\mathrm{nm}$ is attributed to the BODIPY. 

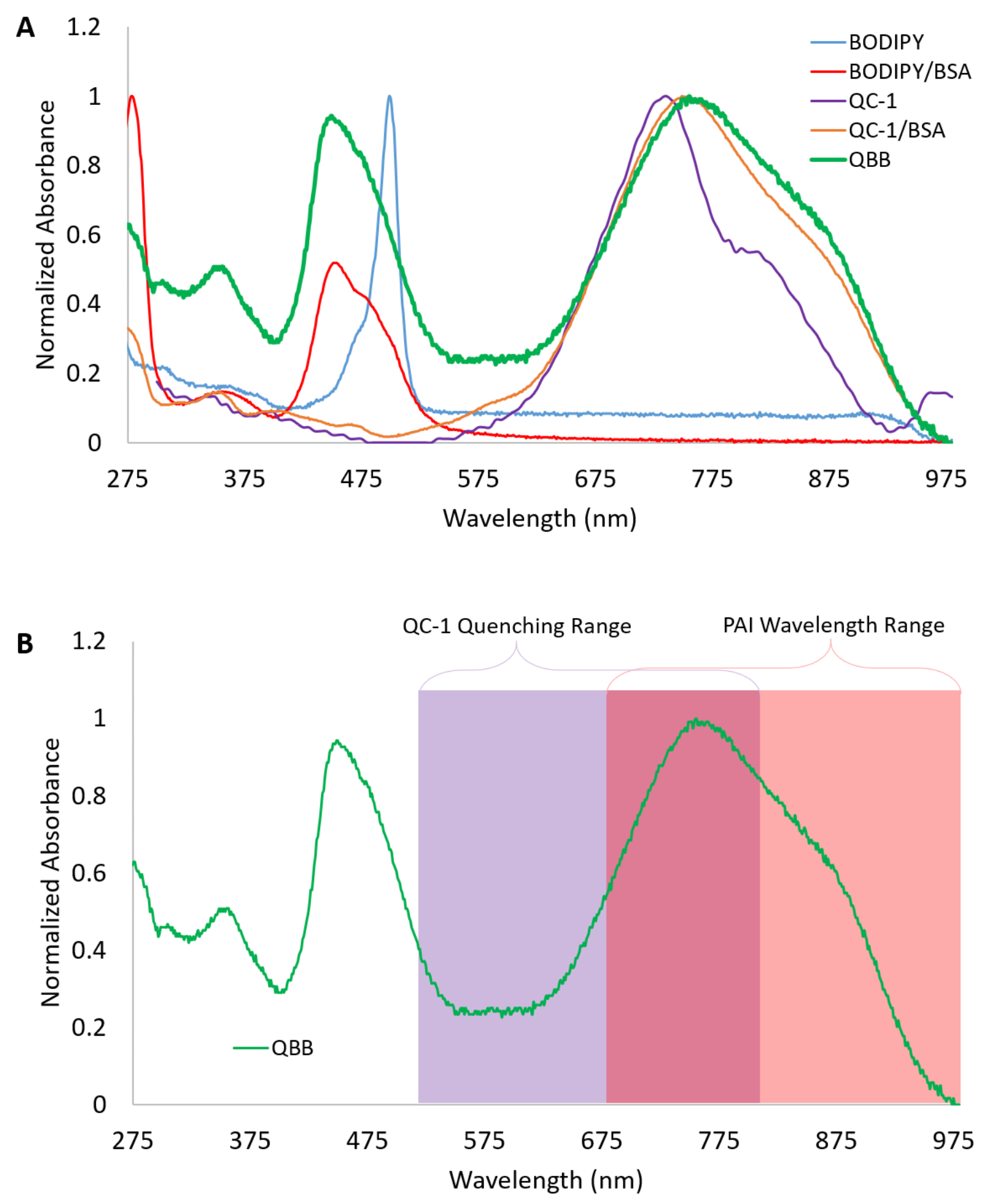

Figure 3-2. The normalized absorbance spectrum of A) free QC-1 (purple) and BODIPY (blue), single dye bound BSA (orange and red respectively), and the QBB tracer (green), and B) The QBB tracer alone. Semi-transparent purple is the quenching range of $Q C-1$ and semi-transparent red is the imaging range of the MSOT PA imager. 
The linear range of concentrations for the QBB tracer was found between 0 and $73 \mu \mathrm{M}$ as shown in Figure 3-3.

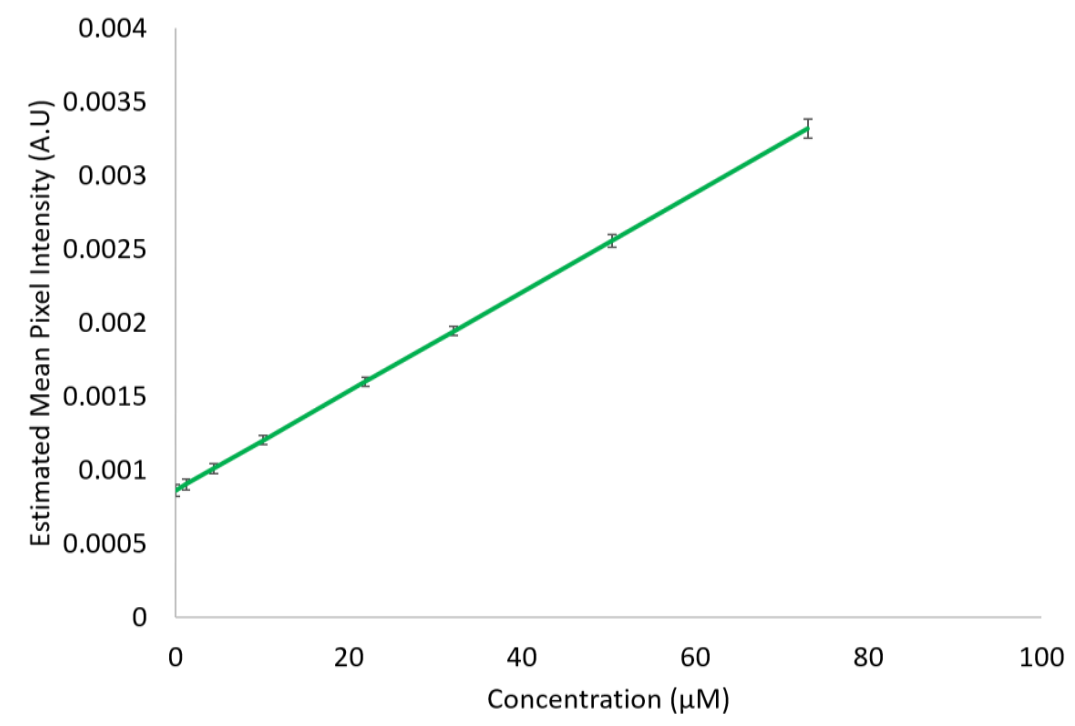

Figure 3-3. The linear range. The range of concentrations where the relationship between the QBB concentration (determined by the concentration of QC-1) and the photoacoustic detection is statistically linear. Error bars the indicate the standard deviation of the three trials. A.U. arbitrary unit.

The minimum detectable concentration graph visualization is shown in Figure 3-4 where QBB has a calculated MDC of $1.22 \pm 1.5 \mu \mathrm{M}$. 


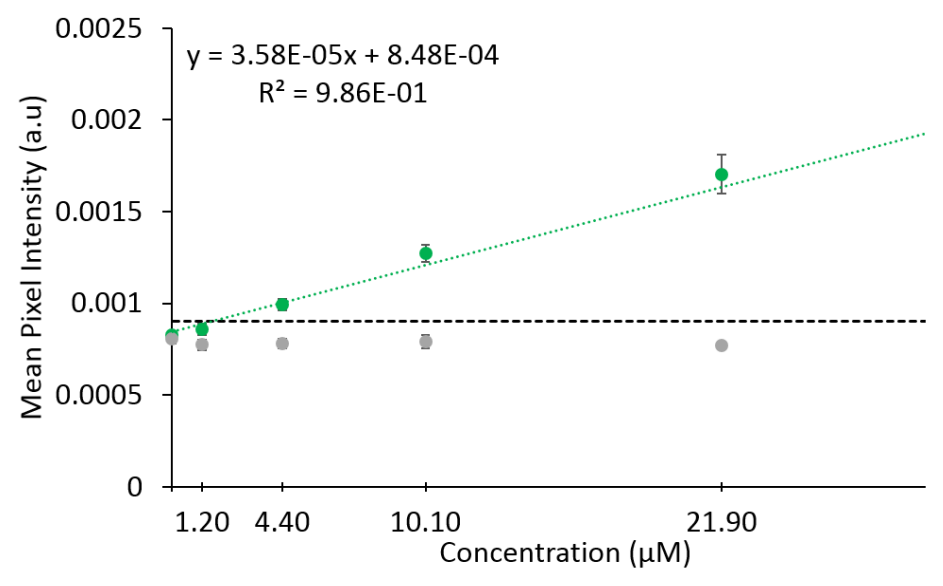

Figure 3-4. The graphical representation of the minimum detectable concentration for the QBB tracer. The green data points represent the average mean pixel intensity of the photoacoustic signal of QBB. Error bars are the standard deviation. The black line is the limit of detection, and the green line is the line of best fit. The intersection between the green and black lines is the minimum detectable concentration (1.22 $\mu M)$. A.U. arbitrary unit.

After one hour of repetitive photoacoustic imaging, the QBB photoacoustic signal showed a decrease of approximately 14\% shown in Figure 3-5.

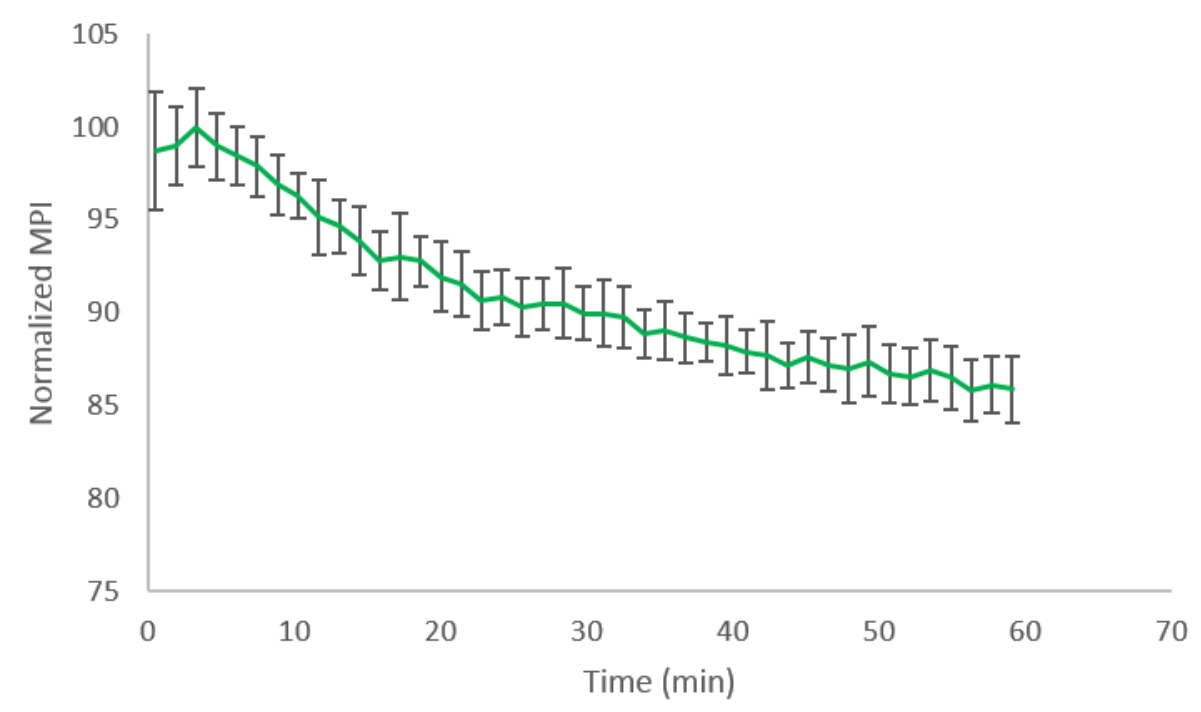

Figure 3-5. The average photoacoustic signal of QBB over time of laser irradiation at $735 \mathrm{~nm}$. Error bars represent the standard deviation between the three samples of $Q B B$. 
Table 5. Fluorescence count reading of QBB with $480 \mathrm{~nm}$ excitation and $520 \mathrm{~nm}$ emission. Values are the average of 3 samples.

\begin{tabular}{lll}
\hline & Without laser irradiation & With 1-hour laser irradiation \\
\hline QBB & 9522 & 12993 \\
\hline
\end{tabular}

\subsection{Discussion}

The development of a hybrid contrast agent for photoacoustic imaging and fluorescence imaging is needed to provide post-mortem validation of the computer-generated PA images. There have been several studies on the development of such a contrast agent. An anti-integrin antibody labeled with ICG developed for dual photoacoustic and fluorescence imaging ${ }^{157}$ was used to image tumor location, angiogenesis, and brain structure in mice. ${ }^{158}$ NIR dye Cy5.5 was used to develop a hybrid photoacoustic and fluorescent tracer to detect tumor-associated mRNA in vivo. ${ }^{153}$ Additionally, nanoparticles loaded with an in-house NIR dye was developed for photoacoustic and fluorescence imaging of tumor-draining sentinel lymph nodes. ${ }^{155}$ All of the aforementioned dual tracers used fluorescent dyes for both the photoacoustic and fluorescence imaging. Since studies have shown that fluorescent proteins ${ }^{166}$ and dyes ${ }^{168}$ tend to show a decrease in photoacoustic signal with increased laser irradiation time, the quantification of the PA images using these contrast agents is challenging. An antibody labeled with two dyes; Black Hole Quencher 3 (BHQ-3) and fluorescein has been demonstrated and shows promise as a hybrid photoacoustic and fluorescent contrast agent. ${ }^{154}$ The BHQ-3 is a non-fluorescent quencher that has a peak absorbance at $672 \mathrm{~nm}$ and quenches dyes that have emission wavelengths between 620 and $730 \mathrm{~nm}$. NHS-fluorescein has a maximum emission wavelength of $518 \mathrm{~nm}$, below the quenching range of BHQ-3. ${ }^{154}$ However, the peak absorption wavelength is below the range of the photoacoustic system they used (680 nm minimum) which reduces its effectiveness as a photoacoustic contrast agent. By developing a hybrid contrast agent using quencher dye QC-1 with a peak wavelength of $737 \mathrm{~nm}$, well within the imaging range of our photoacoustic device (680 nm to 980 $\mathrm{nm})$, the signal detection of the tracer was improved. 
While encapsulation of this highly hydrophobic BOPIPY $493 / 503$ has been seen in lipid droplets, ${ }^{89,} 175$ fluorescent BODIPY nanovesicles, ${ }^{176}$ and nanorods, ${ }^{177}$ to our knowledge this is the first time that BSA has been used as a delivery host for this fluorescent probe through this entropic method of labeling. Other derivatives of BODIPY have been labelled to BSA through several methods including amide bond formation and thiol-ene conjugation, ${ }^{88}$ as well as other proteins. ${ }^{178,} 179$

The photoacoustic characteristics of the hybrid QBB tracer were similar to those of the QC-1/BSA tracer shown in our previous work. ${ }^{168}$ The linear range ( $0-73 \mu \mathrm{M}$ and $0-75 \mu \mathrm{M}$, respectively), minimum detectable concentration $(1.22 \pm 1.5 \mu \mathrm{M}$ and $0.89 \pm 1.28 \mu \mathrm{M}$, respectively) and photoacoustic photobleaching (14\% and 11\% decrease, respectively). The fluorescence of the BODIPY in the QBB tracer is still strong enough to overcome any quenching overlap of the QC-1 dye. Furthermore, the increase in fluorescence signal after repetitive laser exposure indicates that the relative photoacoustic photobleaching of the QC-1 component of the tracer leads to a reduced amount of quenching of the BODIPY component. These in vitro results show promise as a hybrid tracer.

\subsection{Conclusion}

The NIR dark quencher dye QC-1 and fluorescent dye pentamethyl BODIPY were successfully labeled to bovine serum albumin through covalent and entropic labeling respectively. In vitro characterization demonstrated strong photoacoustic signal and strong fluorescence signal. Additional tests in vivo are required to determine feasibility as a tracer for lymphatic drainage in vivo. 


\section{Chapter 4 : Discussion and Conclusion}

In the first half of this thesis, a systematic, non-biased method of characterizing contrast agents for use in photoacoustic imaging was developed based on well-established physics standard methods ${ }^{120,134}$. These methods used statistical analyses to determine the minimum detectable concentration, or sensitivity, of a contrast to a photoacoustic device as well as the linear, or quantifiable, range of concentrations. Furthermore, the photostability, a measurement of a change in photoacoustic signal generation as a function of laser exposure, or, PAI time, that has been performed before in various other studies, ${ }^{113-115}$ was also used to characterize specific contrast agents. Of the three dyes characterized in this thesis, none appeared to have a noticeable difference in the minimum detectable concentration that would prevent any one of the dyes to be used as a signaling compound. The two fluorescent dyes, IRDye800CW and CF770 both have larger linear ranges than the dark quencher QC-1, indicating a larger range for which data can be quantified. However, the steeper slope of the QC-1 linear range indicates that the differentiation between similar concentrations will be more noticeable than the two fluorescent dyes. Furthermore, QC-1's linear range coincided with its minimum detectable concentration. This indicates that QC-1's minimum detectable concentration is equal to the minimum quantifiable concentration which is not the case for the two fluorescent dyes. Furthermore, QC-1 showed the least decay in photoacoustic signal intensity with prolonged laser exposure suggesting that QC-1 is a more photostable dye than the two fluorescent dyes which confirms the conclusions made in a similar study comparing fluorescent and non-fluorescent proteins. ${ }^{166}$

Our goal is to create a hybrid contrast agent for both in vivo quantitative photoacoustic imaging and postmortem fluorescence imaging validation of the lymphatic drainage from the eye in mice. This requires the use of a photoacoustic contrast agent that can withstand multiple imaging sessions and has a linear range that can be used to quantify the photoacoustic signal of the tracer in the lymph nodes. Although QC-1 has the smallest linear range, its minimum detectable concentration being the lower limit of the range allows 
for quantification of the tracer in the lymph nodes. Furthermore, its high photostability compared to the two fluorescent dyes makes it a better signaling compound for photoacoustic imaging at multiple time points after contrast agent injection. Despite this, QC-1 is the only dye of the three that cannot be used for fluorescence imaging and therefore cannot be used as a contrast agent for fluorescence validation purposes. Therefore, to maintain the photoacoustic characteristics of the QC-1 dye as well as to add fluorescent properties to the hybrid tracer, an additional fluorescent dye needs to be added to the targetting ligand. A NIR fluorescent dye like CF770 or IRDye $800 \mathrm{CW}$ could be used; however, the change to the absorption properties in the NIR wavelength range could potentially reduce the tracer's effectiveness by reduced photostability as a photoacoustic contrast agent. Furthermore, the quenching properties of the QC-1 may completely prevent any fluorescence. ${ }^{76}$ To work around the quenching of the QC-1, a fluorescent dye that emits light in the visible range was used for the hybrid tracer.

In the second half of this thesis, the lymph targeting protein albumin was labelled with NIR QC-1 quencher dye and fluorescent dye pentamethyl BODIPY which fluoresces at around $500 \mathrm{~nm}$, outside the quenching range of QC-1 and outside of the range of the laser used for PAI. Photoacoustic characterization yielded similar results to the QC-1 dye alone, and the hybrid tracer showed detectable fluorescence signal. Furthermore, the fluorescence signal of the tracer increased when the tracer was exposed to repetitive photoacoustic imaging. This may coincide with the slight photoacoustic photobleaching (decay in photoacoustic signal) of the QC-1, reducing any potential quenching effect it has on the BODIPY.

A systematic and statistical approach was described for use on photoacoustic contrast agents for the first time and was used to compare three NIR dyes. Of the three dyes, the dark quencher QC-1 was selected for having characteristics desirable for in vivo lymphatic photoacoustic imaging. Dark quencher QC-1 and the fluorescent dye BODIPY were used successfully to dual label to serum albumin to target the lymph nodes and analysis showed promise as a hybrid tracer for in vivo photoacoustic lymphatic imaging paired with fluorescence imaging validation in the same animals. 


\section{Chapter 5 : Future Directions}

In vivo experiments to test the hybrid photoacoustic and fluorescent tracer, $\mathrm{QBB}$, are required to determine whether it can be detected with photoacoustic imaging in vivo and with various fluorescent imaging modalities post-mortem as predicted based on our previous experiments. ${ }^{17}$ Several additional experiments need to be performed prior to the hybrid QBB tracer being used as a contrast agent for lymphatic photoacoustic imaging and fluorescent validation. First, the tracer needs to be considered stable and not deteriorate within physiological conditions. A Western blot of QBB harvested from the lymph nodes can be used to determine the size of the tracer and whether the tracer has been broken into its dye and protein components. If the tracer is shown to not be stable, thiol-reactive BODIPY should be considered for stronger covalent bonding to the tracer since previous studies demonstrated the bond between QC-1 and BSA is stable in physiological conditions. ${ }^{17}$

Additionally, the distribution and life-time of the tracer should be measured in other various organs and signs of toxicity should be monitored at various time points after injection.

\subsection{Materials and Methods}

\subsubsection{Animal Handling}

Mouse experiments were approved by the Animal Care Committee at St. Michael's Hospital. Mice were kept on a 12-hour light/dark cycle with ad libitum food and water. Three CD-1 albino mice (3 female; Charles River Laboratories, QC, Canada) under general anesthesia of 2\% isoflurane and $100 \%$ oxygen had their head and neck regions gently shaved with a hair trimmer (ChroMini, WAHL, IL). Hair removal cream (Nair, Chruch \& Dwight, ON, Canada) was applied for 30 seconds to remove the remaining hair. 


\subsubsection{Mouse Eye Injection}

Prior to injection, using a surgical microscope (Zeiss OPMI 1, Carl Zeiss, Oberkochen, Germany), a right peripheral temporal corneal paracentesis was performed to release approximately $2 \mu \mathrm{L}$ of aqueous humor from the mouse eye. This was to reduce the change in intraocular pressure that would occur after adding 2 $\mu \mathrm{L}$ of $535 \mu \mathrm{M}$ of QBB. Assuming the dye distributes homogenously within the eye, the resulting concentration QBB is approximately $153 \mu \mathrm{M}$ in the eye based on a $7 \mu \mathrm{L}$ anterior chamber in mice. ${ }^{180}$

Mice were injected into the right anterior chamber through the temporal cornea with a 33-G needle on a 10 uL NanoFil Syringe (World Precision Instruments, Sarasota, FL, USA). After injection of the QBB, the cornea was rinsed with artificial tear drops (GenTeal, Alcon, ON, Canada). The mouse eye was then examined under the operating microscope for hemorrhaging or evidence of intraocular injury. A rebound tonometer (TONOLAB, Icare Finland Oy, Vantaa, Finland) was used to measure the intraocular pressure immediately before and after injection. Measurements were taken under the surgical microscope by applying the tonometer perpendicular to the central corneal surface.

\subsubsection{Photoacoustic Imaging}

Mice were imaged under general anesthesia with the same photoacoustic system with wavelengths 680 , $695,735,755,775,795,825,885,925,955$, and $980 \mathrm{~nm}$. Ten frames per wavelength were captured at each scanning position which occurred every $0.5 \mathrm{~mm}$ from the eyes to the neck region. This region is $22 \mathrm{~mm}$ long along the Z-axis where the total imaging time per session is approximately 10 minutes. Mice were imaged prior to injection, as well as $20 \mathrm{~min}, 2$ hours, 4 hours, and 6 hours post-injection. Between imaging sessions, mice were removed from the imaging chamber and animal holder to recover from the anesthesia in a clean cage placed over a heating pad (E-Z Anesthesia, PA) before returning to the home cage with $\mathrm{ad}$ libitum food and water. Mice were sacrificed after the last imaging session for post-mortem fluorescence analysis. 


\subsubsection{Photoacoustic Imge Analysis}

Mouse scans were analyzed using the native viewMSOT software. Scans were reconstructed with the Backprojection algorithm and spectrally unmixed using the Adaptive Match Filter (AMF) to locate the QBB signal. ${ }^{17}$

\subsubsection{Ex Vivo In Situ Fluorescence Imaging}

Mice were sacrificed using $2 \mathrm{~mL}$ of T-61 Euthanasia solution (Merck, NJ, USA) injected into the heart. The skin and fat were removed from the head and neck prior to imaging with a confocal scanning laser ophthalmoscopy system (Spectralis, Heidelberg Engineering, Heidelberg, Germany). Both the eyes and the neck were imaged with Blue Autofluorescence (488 nm excitation, $500 \mathrm{~nm}$ barrier filter) and Infrared reflectance $(820 \mathrm{~nm})$ imaging modes.

\subsubsection{Tissue Preparation}

Mouse head and neck tissue, as well as the right submandibular lymph node, were fixed in $2 \% \mathrm{PFA}$ at $4^{\circ} \mathrm{C}$ for 24 hours after dissection. After three 10 minute PBS washes, the mouse tissue was cryoprotected by immersion in 10\% sucrose (Thermo Fisher Scientific) in PBS for 24 hours, and 20\% sucrose in PBS for 48 hours at $4{ }^{\circ} \mathrm{C}$. The mouse head and neck regions were dissected and the right and left eyes and orbits as well as the right and left cervical lymph nodes were removed and frozen in isopentane (Thermo Fisher Scientific) cooled by dry ice and embedded into cryomatrix (Tissue-Tek O.C.T. Compound, Sakura, South Holland, Netherlands). Tissue blocks were serially sectioned to $20 \mu \mathrm{M}$ thick using a cryostat (Leica CM1900, Leica Biosystems, ON, Canada). Sections were mounted onto charged microscope slides (Superfrost Plus, Thermo Fisher Scientific) and stored at $-20^{\circ} \mathrm{C}$. Sections were counterstained with DAPI nuclear stain (1:5000 dilution; Invitrogen). 


\subsubsection{Fluorescence Imaging of Tissue Sections}

Tissue blocks were serially sectioned using a cryostat (Leica CM1900, Leica Biosystems, ON, Canada) to $20 \mu \mathrm{m}$-thick sections. Sections were mounted onto charged microscope slides (Superfrost Plus, Thermo Fisher Scientific) and stored at $-20^{\circ} \mathrm{C}$. Sections were counterstained with DAPI nuclear stain (1:5000 dilution; Invitrogen). Stained sections of the right and left eyes and cervical lymph nodes were imaged with a confocal laser microscope (LSM700, Zeiss, Oberkochen, Germany) at 20x magnification.

\subsection{Results}

\subsubsection{In Vivo Results}

Strong photoacoustic signal of the QBB tracer was detected in the eye at 20 minute after injection for all three mice as shown in Figure 5-1. Over time, the QBB signal decreased in the eye but was still detected at the 6 hour time point for all mice. The QBB signal was detected in the region of the right cervical lymph node for all three mice as shown in Figure 5-2. The trace amounts of QBB signal was initially detected at varied time points in the right cervical lymph node for each mouse. QBB was detected as early as 2 hours for M2 and as late as 6 hours for M3. M1 showed detection in the right cervical lymph at 4 hours postinjection and two distinct regions are shown and potentially represent tracer in two adjacent lymph nodes. 


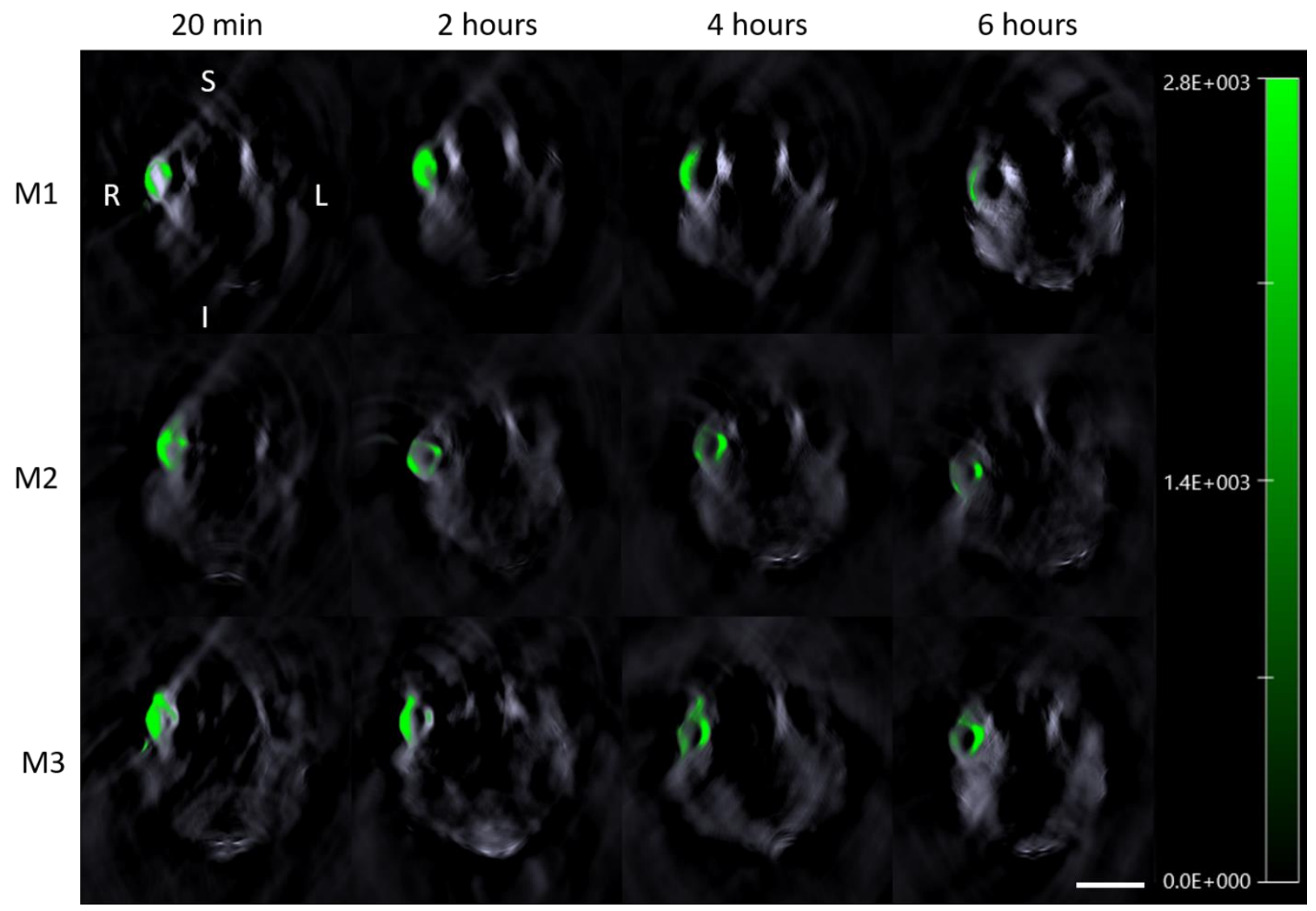

Figure 5-1. Photoacoustic images of the mouse head cross-section at the region of the eyes after injection of $Q B B$ tracer. The top row is mouse M1, the second row is M2, and the third row is M3. Green represents the photoacoustic signal generated from the $Q B B$ tracer. $S=$ Superior, $R=$ Right, $L=L$ Left, $I=$ Inferior. Scale bar $=5 \mathrm{~mm}$. The vertical bar on the right shows pixel intensity values of the QBB tracer ranging from 0 to 2800 a.u.. 


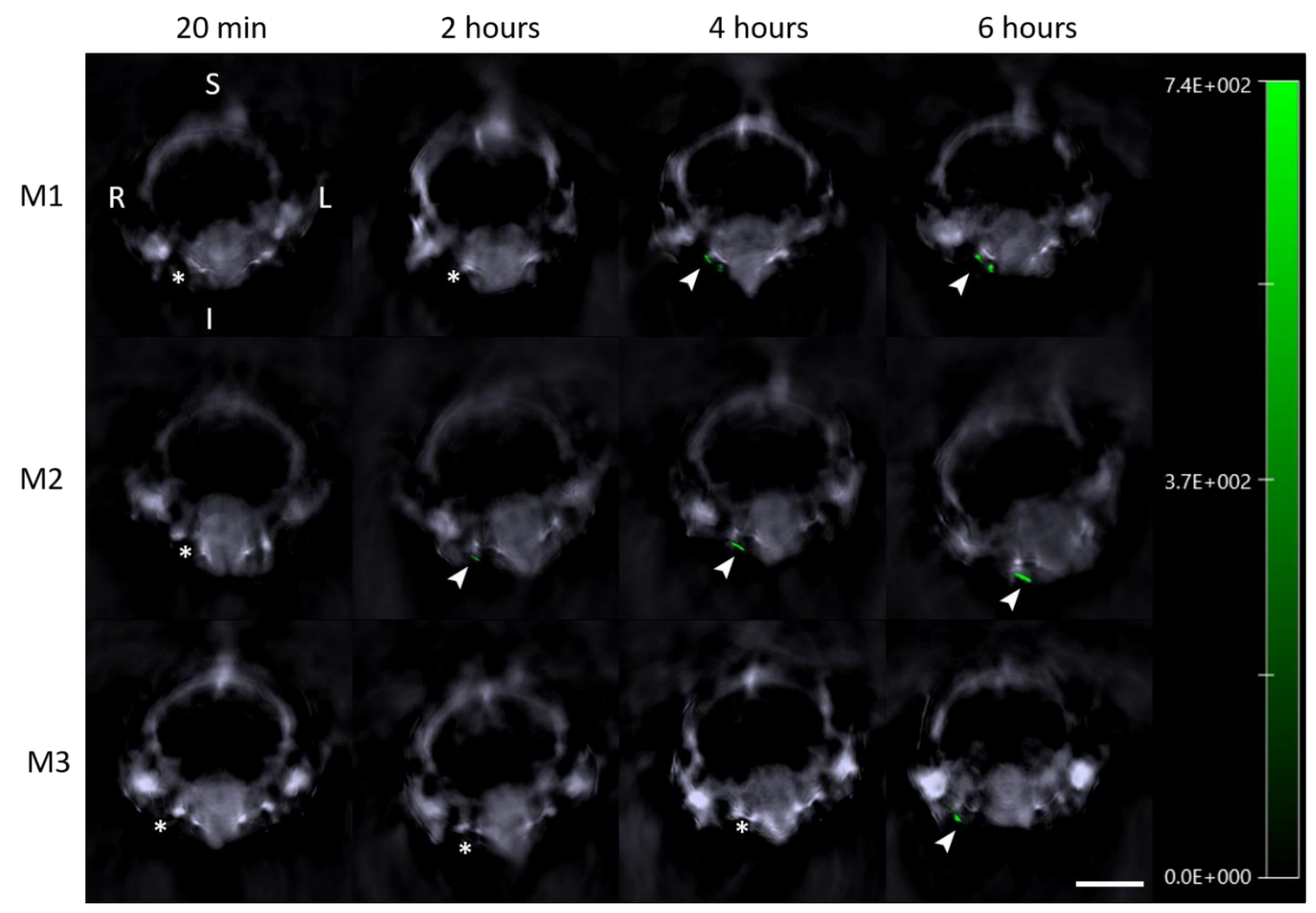

Figure 5-2. Photoacoustic images of the mouse head cross-section at the region of the neck and cervical lymph nodes after injection of $Q B B$ tracer. The top row is mouse M1, the second row is M2, and the third row is M3. Green indicates the $Q B B$ tracer signal. $S=$ Superior, $R=R i g h t, L=L e f t, I=I n f e r i o r$. Scale bar $=5 \mathrm{~mm}$. Asterisks indicate the location of right cervical lymph node without tracer signal. Arrows indicate the presence of tracer signal in right cervical lymph node. The vertical bar on the right shows pixel intensity values of the QBB tracer ranging from 0 to 740 a.u.

\subsubsection{Post Mortem Results}

All 3 mice had strong fluorescence signal in their injected right eye (Figure 5-3), however, the tracer signal was not detected in the right cervical lymph nodes. 


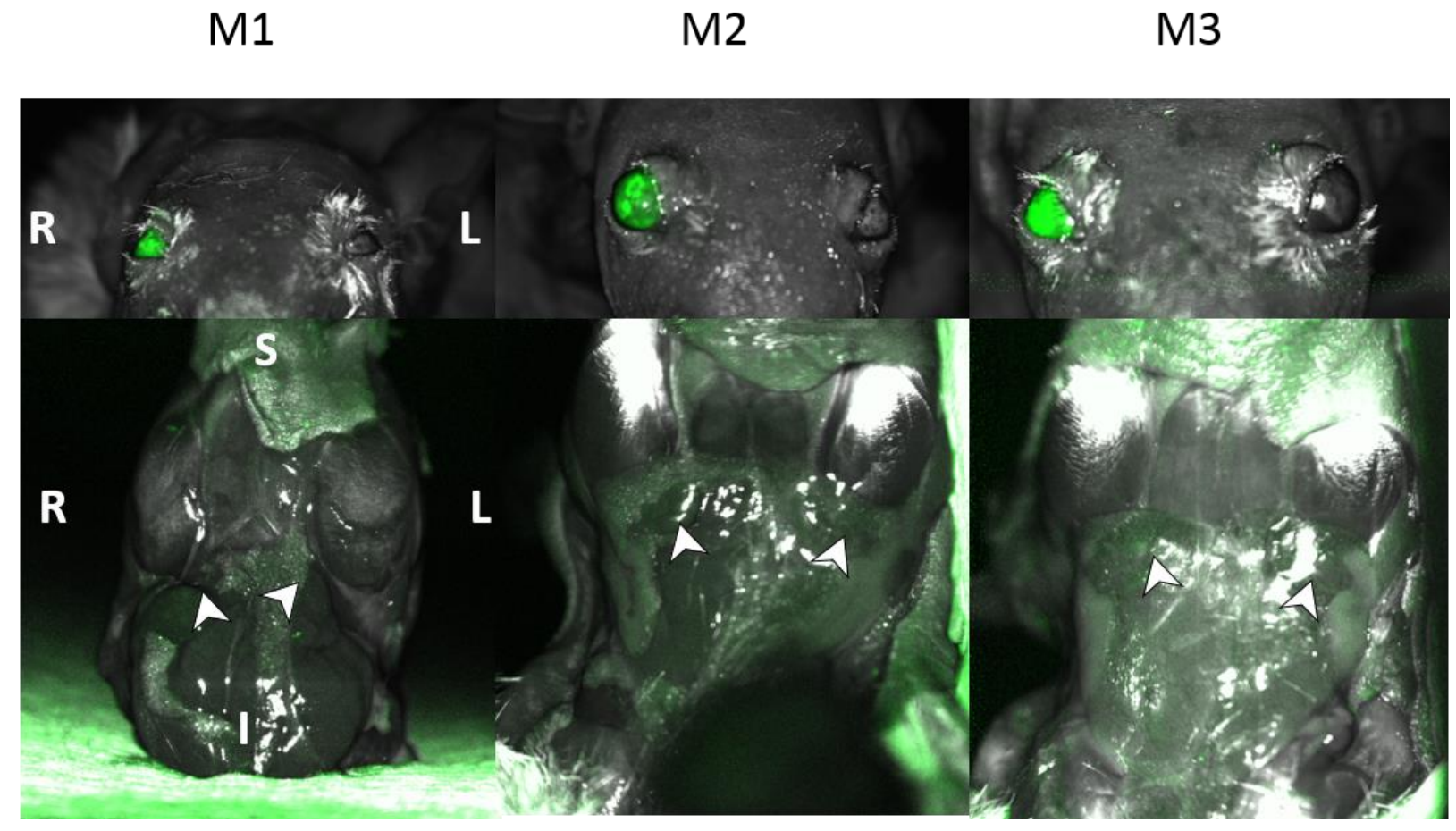

Figure 5-3. Infrared ( $\mathrm{nm}$ ) images of mice heads (top row) and necks (bottom row) with blue autofluorescence (nm) images overlaid in green. The green indicates the tracer in the right eye in all three mice. $R=R i g h t, L=L e f t$. The arrowheads indicate the location of the cervical lymph nodes. M1, M2, and M3 are the mice ID numbers.

Mouse right and left eye histology sections are shown in Figure 5-4. Fluorescence signal within the range of the QBB emission wavelengths was detected in the right eye lens, cornea, retina, ciliary body, and surrounding orbital tissue. This was not seen in the left eye sections. The sections of the right and left cervical lymph nodes showed no fluorescence signal of the tracer (Figure 5-5). 


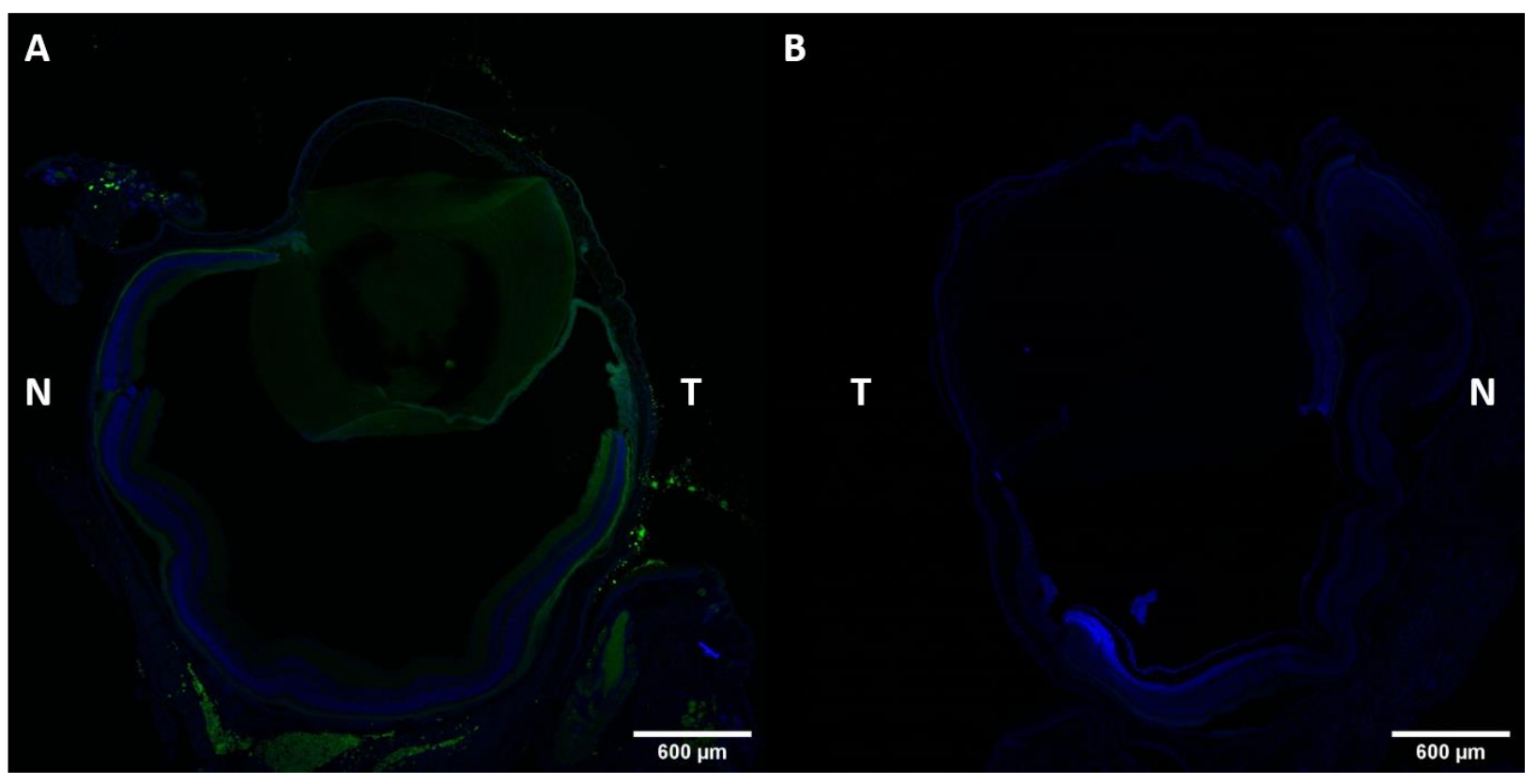

Figure 5-4. Right (injected) eye (A) and left (non-injected) eye (B) histology slide sections of mouse \# M2. Green is the tracer, blue is the nuclear stain. $N=$ Nasal and $T=$ Temporal. The scale bar is $600 \mu \mathrm{m}$. 


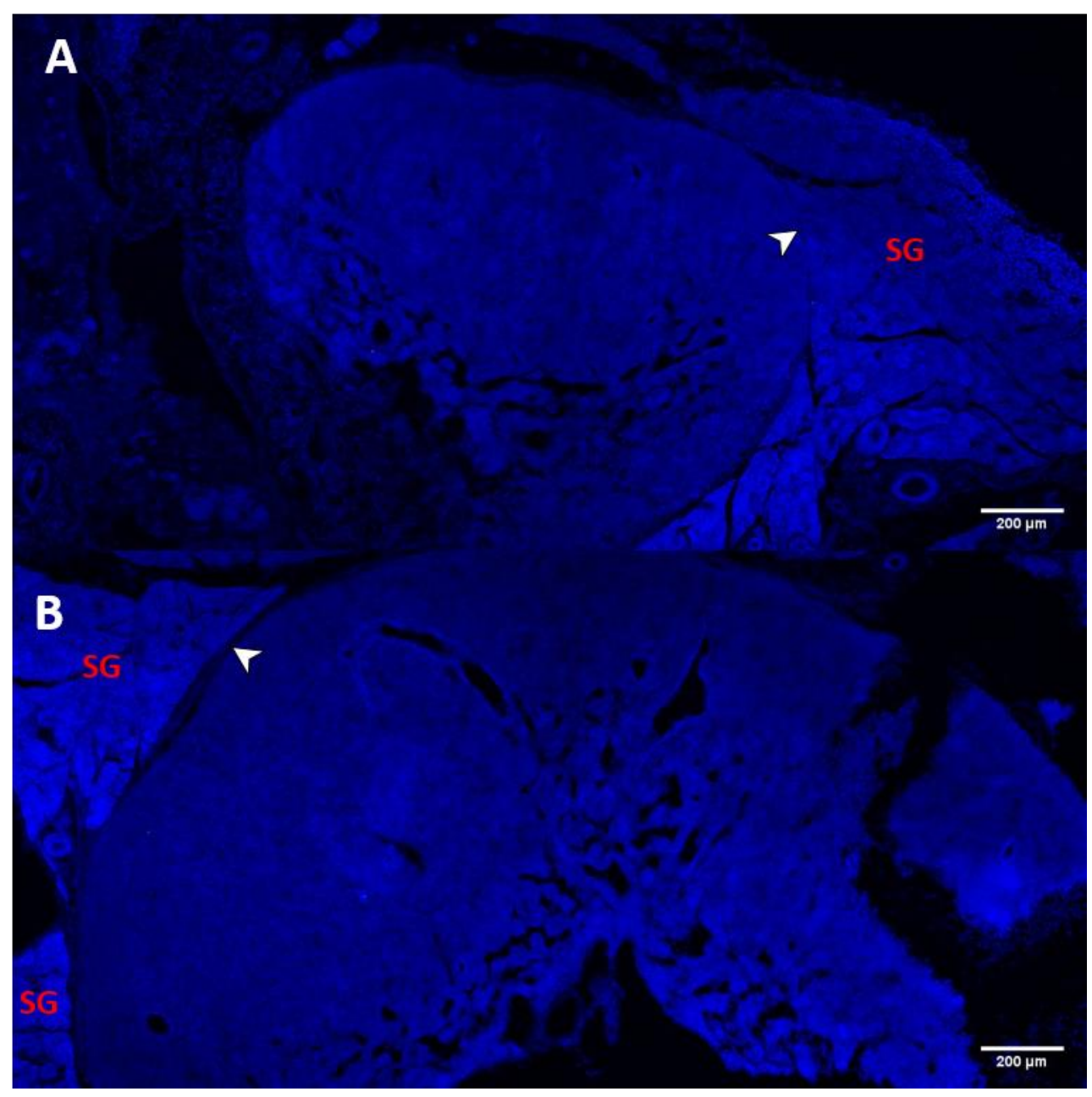

Figure 5-5. Right (A) and left (B) cervical lymph nodes histology slide sections of the mice M2 under confocal laser microscopy. Green is the tracer, blue is the nuclear counterstain with DAPI. No green tracer is noted in both cervical lymph nodes. $S G=$ salivary gland, arrowheads point to lymph node border. The scale bar indicates $200 \mu m$.

\subsection{Discussion and Conclusion}

The in vitro data from Chapter 3 indicates that the concentration injected in the eye $(153 \mu \mathrm{M}$ after dilution in the anterior chamber) is above the linear range of the QBB tracer $(73 \mu \mathrm{M})$. Therefore, despite strong QBB signal in the injected eye for all three mice, the concentration of the dye in the eye was too high to perform accurate quantitative analysis. Upon further evaluation of the fluorescence histology of 3 animals that underwent 4 sessions of PAI after tracer injection into the right eye, we see the fluorescence signal in 
the right eye in the lens and in the deeper layers of the retina, and not in the left eye shown in Error! Reference source not found.. This result suggests that the BODIPY fluorescence signal was detectable after laser irradiation and tissue processing. Due to the large size of BSA and the low permeability of the lens, this finding indicates that there was either a large amount of free BODIPY in the stock tracer solution even after filtration, or, that the BODIPY is becoming unbound from the hybrid tracer in vivo. Since there is a clear spectral change that occurs when BODIPY is bound to BSA, we can assume that the latter is the more likely reason.

When studying lymphatic drainage specifically, the signal of the contrast agent in the lymph node is more relevant. If quantification of aqueous humor drainage regardless of which pathway is taken is desired, then a lower injected concentration in the eye is needed. The in vivo photoacoustic images of the injected mice showed results similar to our previous experiments using a tracer containing only QC-1 conjugated BSA ${ }^{17}$ where the QBB was detected in the right lymph node in all three mice imaged. However, when tested for validation, no fluorescence signal was detected in the right lymph node in the low nor high power confocal scans. Since we were able to validate these same results in our previous study, ${ }^{17}$ we attribute these results to there being a fault in the hybrid tracer.

The relatively weak entropic binding of the BODIPY to the BSA compared to the covalent binding with QC-1 may explain these results. By using a BODIPY dye that can covalently bind to BSA without competing against the QC-1 binding may improve the post-mortem fluorescence imaging validation required for the lymphatic drainage. The BODIPY molecule we used was the lab-made version of the pentamethyl BODIPY dye by Invitrogen (D3922) which is primarily used for lipid binding. We hypothesize that under physiological conditions, stronger entropic forces are present and the affinity of the BODIPY to the BSA is not strong enough to keep the BODIPY bound to the BSA in in vivo conditions. Future experiments using BODIPY forms that covalently bind to proteins such as thiol-reactive BODIPYs (ie BODIPY iodoacetamides, BODIPY maleimides and BODIPY methyl bromides) ${ }^{88,105}$ should be tested for 
double labeling of BSA to determine if the stability of the hybrid tracer withstands physiological conditions while still maintaining photoacoustic and fluorescent properties in vitro.

Despite the promising results of the in vitro characterization of the QBB tracer in Chapter 3, the postmortem fluorescence imaging was not able to validate the photoacoustic signal detected in vivo in the lymph node. Further experimental work adjusting the methods of BODIPY labeling are required to develop a more physically stable, hybrid photoacoustic-fluorescent contrast agent for the use of dynamic lymphatic imaging in vivo. 


\section{References}

J. Xia, J. Yao, and L.H. V. Wang, Photoacoustic tomography: principles and advances (invited review), Prog. Electromagn. Res. 147, 1-22 (2014).

D. Salo, H. Zhang, D.M. Kim, and M.Y. Berezin, Multispectral measurement of contrast in tissuemimicking phantoms in near-infrared spectral range of 650 to $1600 \mathrm{~nm}$, J. Biomed. Opt. 19(8), 086008 (2014).

Y.C. Tham, X. Li, T.Y. Wong, H.A. Quigley, T. Aung, and C.Y. Cheng, Global prevalence of glaucoma and projections of glaucoma burden through 2040: A systematic review and metaanalysis, Ophthalmology 121(11), 2081-2090 (2014).

R.N. Weinreb, T. Aung, and F.A. Medeiros, The pathophysiology and treatment of glaucoma: A review, JAMA - J. Am. Med. Assoc. 311(18), 1901-1911 (2014).

H. Quigley, Glaucoma, Lancet 377(9774), 1367-77 (2011).

M. Goel, R.G. Picciani, R.K. Lee, and S.K. Bhattacharya, Aqueous Humor Dynamics : A Review, $52-59(2010)$.

U.R. Chowdhury, B.J. Madden, M.C. Charlesworth, and M.P. Fautsch, Proteome analysis of human aqueous humor, Investig. Ophthalmol. Vis. Sci. 51(10), 4921-4931 (2010).

K. Inada and E. Al, Increase of aqueous humor proteins with aging, Jpn J Ophthalmol 32(2), 126$131(1988)$.

D. Purves, G.J. Augustine, D. Fitzpatrick, et al., Anatomy of the Eye, in Neuroscience, 2nd ed.(Sinauer Associates, Sunderland (MA), 2001).

M.M. Civan and A.D.C. Macknight, The ins and outs of aqueous humour secretion, 78, 625-631 
(2004).

H.H. Mark, Aqueous Humor Dynamics in Historical Perspective, Surv. Ophthalmol. 55(1), 89-100 (2010).

M. Johnson, J.W. Mclaren, and D.R. Overby, Unconventional aqueous humor out flow : A review, Exp. Eye Res. 158, 94-111 (2017).

A. Alm and S.F.E. Nilsson, Uveoscleral outflow - A review, Exp. Eye Res. 88(4), 760-768 (2009).

Y.H. Yücel, M.G. Johnston, T. Ly, et al., Identification of lymphatics in the ciliary body of the human eye: A novel "uveolymphatic" outflow pathway, Exp. Eye Res. 89(5), 810-819 (2009).

M. Kim, M.G. Johnston, N. Gupta, S. Moore, and Y.H. Yücel, A model to measure lymphatic drainage from the eye, Exp. Eye Res. 93(5), 586-591 (2011).

A.L.C. Tam, N. Gupta, Z. Zhang, and Y.H. Yücel, Quantum dots trace lymphatic drainage from the mouse eye., Nanotechnology 22(42), 425101 (2011).

Y.H. Yücel, K. Cardinell, S. Khattak, et al., Active lymphatic drainage from the eye measured by noninvasive photoacoustic imaging of near-infrared nanoparticles, Investig. Ophthalmol. Vis. Sci. 59(7), 2699-2707 (2018).

H. Quigley and A.T. Broman, The number of people with glaucoma worldwide in 2010 and 2020, Br. J. Ophthalmol. 90(3), 262-267 (2006).

A. Mantravadi and N. Vadhar, Glaucoma, Prim. Care Clin. Off. Pract. 42(3), 437-49 (2015).

C. Review, The Pathophysiology and Treatment of Glaucoma A Review, 311(18), 1901-1911 (2014). 
E. Rulli, E. Biagioli, I. Riva, et al., Efficacy and Safety of Trabeculectomy vs Nonpenetrating Surgical Procedures, JAMA Ophthalmol. 131(12), 1573 (2013).

B. Ann, P.L. Kacfman, and C. Podos, Prostaglandin F2 Increases Uveoscleral Outflow in the Cynomolgus Monkey, Exp Eye Res 389-402 (1989).

A.L.C. Tam, N. Gupta, Z. Zhang, and Y.H. Yücel, Latanoprost Stimulates Ocular Lymphatic Drainage: An In Vivo Nanotracer Study., Transl. Vis. Sci. Technol. 2(5), 3 (2013).

M. Aihara, J.D. Lindsey, and R.N. Weinreb, Aqueous Humor Dynamics in Mice, Investig. Ophthalmol. Vis. Sci. 44(12), 5168-5173 (2003).

M. Aihara, J.D. Lindsey, and R.N. Weinreb, Reduction of intraocular pressure in mouse eyes treated with latanoprost, Investig. Ophthalmol. Vis. Sci. 43(1), 146-150 (2002).

J.D. Lindsey and R.N. Weinreb, Identification of the mouse uveoscleral outflow pathway using fluorescent dextran, Investig. Ophthalmol. Vis. Sci. 43(7), 2201-2205 (2002).

W.. Generoso, Mouse models for understanding human developmental anomalies, Prog. Clin. Biol. Res. 340C, 151-157 (1990).

S. Karaman, D. Buschle, P. Luciani, J.C. Leroux, M. Detmar, and S.T. Proulx, Decline of lymphatic vessel density and function in murine skin during aging, Angiogenesis 18(4), 489-498 (2015).

M. Ishikawa, T. Yoshitomi, C.F. Zorumski, and Y. Izumi, Experimentally Induced Mammalian Models of Glaucoma, Biomed Res. Int. 2015, 1-11 (2015).

T. V. Johnson and S.I. Tomarev, Rodent models of glaucoma, Brain Res. Bull. 81(2-3), 349-358 (2010). 
S.W. John, R.S. Smith, O. V Savinova, et al., Essential iris atrophy, pigment dispersion, and glaucoma in DBA/2J mice [published erratum appears in Invest Ophthalmol Vis Sci 1998 Aug;39(9):1641], Invest Ophthalmol Vis Sci 39(6), 951-962 (1998).

J. Grabska, R.H. Anderson, M. Loukas, J. Birungi, N. Abel, and R. Shane Tubbs, The cardiac lymphatic system (2011).

P. Moriguchi, P. Sannomiya, P.F. Lara, R.M. Oliveira-Filho, K. V. Greco, and L.S. Sudo-Hayashi, Lymphatic system changes in diabetes mellitus: Role of insulin and hyperglycemia, Diabetes. Metab. Res. Rev. 21(2), 150-157 (2005).

V. Mumprecht and M. Detmar, Lymphangiogenesis and cancer metastasis, J. Cell. Mol. Med. 13(8 A), 1405-1416 (2009).

J. Wilting, J. Becker, K. Buttler, and H. Weich, Lymphatics and Inflammation, Curr. Med. Chem. 16(34), 4581-4592 (2009).

N.L. Harvey, R.S. Srinivasan, M.E. Dillard, et al., Lymphatic vascular defects promoted by Prox1 haploinsufficiency cause adult-onset obesity, Nat. Genet. 37(10), 1072-1081 (2005).

T. Barrett, P.L. Choyke, and H. Kobayashi, Imaging of the lymphatic system: new horizons, Contrast Media Mol. Imaging 1(6), 230-245 (2006).

R.T. Lucarelli, M. Ogawa, N. Kosaka, B. Turkbey, H. Kobayashi, and P.L. Choyke, New Approaches to Lymphatic Imaging, Lymphat. Res. Biol. 7(4), 205-214 (2009).

40

S.K. Mouli, L.C. Zhao, R.A. Omary, and C.S. Thaxton, Lymphotropic nanoparticle enhanced MRI for the staging of genitourinary tumors, Nat. Rev. Urol. 7(2), 84-93 (2010). 
Flow Using Removal Rate Constants of Interstitial Macromolecules: A Critical Review of Lymphoscintigraphy, Lymphat. Res. Biol. 5(3), 183-202 (2007).

A. Sherman and M. Ter-Pogossian, Lymph node concentration of radioactive colloidal gold following interstitial injection, Cancer 6(6), 1238-1240 (1953). dextran for mammary lymphoscintigraphy in dogs, Vet. Radiol. Ultrasound 49(5), 487-491 (2008). autofluorescence from paraffin-embedded, formalin-fixed tissue sections, Photonic Appl. Biosensing Imaging 5969(October 2005), 59692E (2005).

A.C. Croce and G. Bottiroli, Autofluorescence spectroscopy and imaging: a tool for biomedical research and diagnosis, Eur. J. Histochem. 58(4), (2014).

N. Teraphongphom, C.S. Kong, J.M. Warram, and E.L. Rosenthal, Specimen mapping in head and neck cancer using fluorescence imaging, Laryngoscope Investig. Otolaryngol. 2(6), 447-452 (2017).

H. Wu, X. Xu, H. Ying, et al., Preliminary study of indirect CT lymphography-guided sentinel lymph node biopsy in a tongue VX2 carcinoma model, Int. J. Oral Maxillofac. Surg. 38(12), 1268$1272(2009)$.

A. Ruddell, M.I. Harrell, S. Minoshima, et al., Dynamic Contrast-Enhanced Magnetic Resonance Imaging of Tumor-Induced Lymph Flow, Neoplasia 10(7), 706-IN4 (2015).

49

S.M. Daly and M.J. Leahy, 'Go with the flow ': A review of methods and advancements in blood flow imaging, J. Biophotonics 6(3), 217-255 (2013). 
Optical + Biomedical Engineering Laboratory, Introduction to OCT, (n.d.).

J. Weber, P.C. Beard, and S.E. Bohndiek, Contrast agents for molecular photoacoustic imaging, Nat. Methods 13(8), 639-650 (2016).

F. Zhang, G. Niu, G. Lu, and X. Chen, Preclinical lymphatic imaging, Mol. Imaging Biol. 13(4), 599-612 (2011).

G. Ravizzini, B. Turkbey, T. Barrett, H. Kobayashi, and P.L. Choyke, Nanoparticles in sentinel lymph node mapping, Wiley Interdiscip. Rev. Nanomedicine Nanobiotechnology 1(6), 610-623 (2009).

T. Jones, The imaging science of positron emission tomography, Eur. J. Nucl. Med. 23(7), 807-813 (1996).

Y.-J. Lee, E.-J. Jeong, H.-W. Song, et al., Photoacoustic imaging probe for detecting lymph nodes and spreading of cancer at various depths, J. Biomed. Opt. 22(9), 091513 (2017).

C. Lee, J. Kim, Y. Zhang, et al., Dual-color photoacoustic lymph node imaging using nanoformulated naphthalocyanines, Biomaterials 73, 142-148 (2015).

D. Pan, X. Cai, C. Yalaz, et al., Photoacoustic sentinel lymph node imaging with self-assembled copper neodecanoate nanoparticles, ACS Nano 6(2), 1260-1267 (2012).

X. Cai, W. Li, C.-H. Kim, Y. Yuan, L. Wang, and Y. Xia, In Vivo Quantitative Evaluation of the Transport Kinetics of Gold Nanocages in a Lymphatic System by Noninvasive Photoacoustic Tomography, ACS Nano 5(12), 9658-9667 (2011).

A.G. Bell, Upon the production and reproduction of sound by light, J. Soc. Telegr. Eng. 9(34), 404426 (1880). 
L. V. Wang and C. Li, Photoacoustic tomography and sensing in biomedicine, Phys. Med. Biol. 54(19), R59-R97 (2009).

S. Tzoumas and V. Ntziachristos, Spectral unmixing techniques for optoacoustic imaging of tissue pathophysiology., Philos. Trans. A. Math. Phys. Eng. Sci. 375(2107), 20170262 (2017).

D.C. Heinz and C.I. Chang, Fully constrained least squares linear spectral mixture analysis method for material quantification in hyperspectral imagery, IEEE Trans. Geosci. Remote Sens. 39(3), 529$545(2001)$.

N. Keshava, A Survey of Spectral Unmixing Algorithms, Lincoln Lab. J. 14(1), 55-78 (2003).

S. Tzoumas, N. Deliolanis, S. Morscher, and V. Ntziachristos, Unmixing molecular agents from absorbing tissue in multispectral optoacoustic tomography, IEEE Trans. Med. Imaging 33(1), 4860 (2014).

S. Roberts, C. Andreou, C. Choi, et al., Sonophore-enhanced nanoemulsions for optoacoustic imaging of cancer, Chem. Sci. 9(25), 5646-5657 (2018).

66 J. Weber, P.C. Beard, and S.E. Bohndiek, Contrast agents for molecular photoacoustic imaging, Nat. Methods 13(8), 639-650 (2016). M.D. Laramie, M.K. Smith, F. Marmarchi, L.R. McNally, and M. Henary, Small molecule optoacoustic contrast agents: An unexplored avenue for enhancing in vivo imaging, Molecules 23(11), 2766 (2018).

68 R. Duncan and R. Gaspar, Nanomedicine(s) under the microscope, Mol. Pharm. 8(6), 2101-2141 (2011). 
nanoparticles, Nanoscale Res. Lett. 4(8), 858-864 (2009).

70

I. Fratoddi, I. Venditti, C. Cametti, and M.V. Russo, How toxic are gold nanoparticles? The stateof-the-art, Nano Res. 8(6), 1771-1799 (2015).

A.M. Alkilany and C.J. Murphy, Toxicity and cellular uptake of gold nanoparticles: What we have learned so far?, J. Nanoparticle Res. 12(7), 2313-2333 (2010).

S. Fraga, A. Brandão, M.E. Soares, et al., Short- and long-term distribution and toxicity of gold nanoparticles in the rat after a single-dose intravenous administration, Nanomedicine Nanotechnology, Biol. Med. 10(8), 1757-1766 (2014).

M. Rathore, I.R. Mohanty, U. Maheswari, N. Dayal, R. Suman, and D.S. Joshi, Comparative in vivo assessment of the subacute toxicity of gold and silver nanoparticles, J. Nanoparticle Res. 16(4), 1$12(2014)$.

C.P. Toseland, Fluorescent labeling and modification of proteins, J. Chem. Biol. 6(3), 85-95 (2013).

B. I., S. A.M., M. R., et al., 99mTc-Human serum albumin: An effective radiotracer for identifying sentinel lymph nodes in melanoma, J. Nucl. Med. 40(7), 1143-1148 (1999).

X. Peng, H. Chen, D.R. Draney, W. Volcheck, A. Schutz-Geschwender, and D.M. Olive, A nonfluorescent, broad-range quencher dye for Förster resonance energy transfer assays, Anal. Biochem. 388(2), 220-228 (2009).

B. Simard, B. Tomanek, F.C.J.M. Van Veggel, and A. Abulrob, Optimal dye-quencher pairs for the design of an "activatable" nanoprobe for optical imaging, Photochem. Photobiol. Sci. 12(10), 18241829 (2013).

J. Zhang, L.P. Smaga, N.S.R. Satyavolu, J. Chan, and Y. Lu, DNA Aptamer-Based Activatable 
Probes for Photoacoustic Imaging in Living Mice, J. Am. Chem. Soc. 139(48), 17225-17228 (2017).

R. Huang, J. Vider, J.L. Kovar, D.M. Olive, and I.K. Mellinghoff, Integrin a v b 3 -Targeted IRDye 800CW Near-Infrared Imaging of Glioblastoma, (7), 5731-5741 (2012).

B. Xie, I.M. Mol, S. Keereweer, et al., Dual-Wavelength Imaging of Tumor Progression by Activatable and Targeting Near-Infrared Fluorescent Probes in a Bioluminescent Breast Cancer Model, 7(2), 1-9 (2012).

J. Joseph, M.R. Tomaszewski, I. Quiros-Gonzalez, J. Weber, J. Brunker, and S.E. Bohndiek, Evaluation of Precision in Optoacoustic Tomography for Preclinical Imaging in Living Subjects, J. Nucl. Med. 58(5), 807-814 (2017).

A.B.E. Attia, C.J.H. Ho, P. Chandrasekharan, et al., Multispectral optoacoustic and MRI coregistration for molecular imaging of orthotopic model of human glioblastoma, J. Biophotonics 9(7), 701-708 (2016).

S.T. Proulx, P. Luciani, A. Alitalo, et al., Non-invasive dynamic near-infrared imaging and quantification of vascular leakage in vivo, Angiogenesis 16(3), 525-540 (2013).

M.L. Landsman, G. Kwant, G.A. Mook, and W.G. Zijlstra, Light-absorbing properties, stability, and spectral stabilization of indocyanine green, J. Appl. Physiol. 40(4), 575-583 (1976).

R.K. Nicholes, Studies on Indocyanine Green Dye Solution, 29(2), 2-6 (1970).

A. Loudet and K. Burgess, BODIPY Dyes and Their Derivatives: Syntheses and Spectroscopic Properties, Chem. Rev. 107(11), 4891-4932 (2007).

Y. Chen, K. Tsao, S.L. Acton, and J.W. Keillor, A Green BODIPY-Based, Super-Fluorogenic, Protein-Specific Labelling Agent, Angew. Chemie - Int. Ed. 57(38), 12390-12394 (2018). 
D. Kim, D. Ma, M. Kim, et al., Fluorescent Labeling of Protein Using Blue-Emitting 8-AminoBODIPY Derivatives, J. Fluoresc. 27(6), 2231-2238 (2017).

E.E. Spangenburg, S.J.P. Pratt, L.M. Wohlers, and R.M. Lovering, Use of BODIPY (493/503) to Visualize Intramuscular Lipid Droplets in Skeletal Muscle, J. Biomed. Biotechnol. 2011, 1-8 (2011). BODIPY Dye Series - Section 1 . 4, Thermo Fish. Sci. (n.d.).

A.K. Wright and M.R. Thompson, Hydrodynamic structure of bovine serum albumin determined by transient electric birefringence., Biophys. J. 15(2 Pt 1), 137-41 (1975).

R. Spinella, R. Sawhney, and R. Jalan, Albumin in chronic liver disease: structure, functions and therapeutic implications, Hepatol. Int. 10(1), 124-132 (2016).

H. Maeda and Y. Matsumura, A new concept for macromolecular therapeutics in cancer chemotherapy: mechanism of tumoritropic accumulation of proteins and the antitumor agent smancs, Cancer Res. 46(12 Pt 1), 6387-6392 (1986).

H. Maeda, Y. Miyamoto, L.W. Seymour, and L.W. Seymour, Conjugates of Anticancer Agents and Polymers: Advantages of Macromolecular Therapeutics in Vivo, Bioconjug. Chem. 3(5), 351-362 (1992). Y. Akdogan, Y. Wu, K. Eisele, M. Schaz, T. Weil, and D. Hinderberger, Host-guest interactions in polycationic human serum albumin bioconjugates, Soft Matter 8(43), 11106 (2012).

M. Biochemistry, D.R. January, and A. May, Evidence for a Large and Flexible Region of Human Serum Albumin Possessing High Affinity Binding Sites for Salicylate, Warfarin, and Other Ligands, 160-171 (n.d.). 
R. Kuhne, R. Franke, H. Sprinz, and G. Hubner, Hydrophobic binding of phenoxyacetic and phenylacetic acids to horseradish peroxidase and human serum albumin: Structure-activity relationships, Acta Biol. Med. Ger. 38(10), 1441-6 (1979).

B.A. Russell, K. Kubiak-Ossowska, P.A. Mulheran, D.J.S. Birch, and Y. Chen, Locating the nucleation sites for protein encapsulated gold nanoclusters: A molecular dynamics and fluorescence study, Phys. Chem. Chem. Phys. 17(34), 21935-21941 (2015).

M. Yang, S. Hoppmann, L. Chen, and Z. Cheng, Human Serum Albumin Conjugated Biomolecules for Cancer Molecular Imaging, Curr. Pharm. Des. 18(8), 1023-1031 (2012).

K. Kanazaki, K. Sano, A. Makino, et al., Development of human serum albumin conjugated with near-infrared dye for photoacoustic tumor imaging, J. Biomed. Opt. 19(9), 096002 (2014).

Q. Chen, X. Liu, J. Chen, J. Zeng, Z. Cheng, and Z. Liu, A Self-Assembled Albumin-Based Nanoprobe for in Vivo Ratiometric Photoacoustic pH Imaging, Adv. Mater. 27(43), 6820-6827 (2015).

Q. Jia, J. Ge, W. Liu, et al., Biocompatible Iron Phthalocyanine-Albumin Assemblies as Photoacoustic and Thermal Theranostics in Living Mice, ACS Appl. Mater. Interfaces 9(25), 21124-21132 (2017).

C. Sim, H. Kim, H. Moon, H. Lee, J.H. Chang, and H. Kim, Photoacoustic-based nanomedicine for cancer diagnosis and therapy, J. Control. Release 203, 118-125 (2015).

J. Steinhard, J. Krijn, and J.G. Leidy, Differences between Bovine and Human Serum Albumins: Binding Isotherms, Optical Rotatory Dispersion, Viscosity, Hydrogen Ion Titration, and Fluorescence Effects, Biochemistry 10(22), 4005-4015 (1971).

M.P. Handbook, F. Fundamentals, T.A. Derivatives, et al., Thiol-Reactive Probes Excited with 
Visible Light, (2019).

J. Shi, Y. Tang, and J. Yao, Advances in super-resolution photoacoustic imaging, Quant. Imaging Med. Surg. 8(8), 724-732 (2018).

H.F. Zhang, K. Maslov, G. Stoica, and L. V. Wang, Functional photoacoustic microscopy for highresolution and noninvasive in vivo imaging, Nat. Biotechnol. 24(7), 848-851 (2006).

L. Lin, P. Zhang, S. Xu, et al., Handheld optical-resolution photoacoustic microscopy, Opt. Express 22(4), 20097 (2017).

C. Kim, S. Park, J. Kim, et al., Objective-free optical-resolution photoacoustic microscopy, J. Biomed. Opt. 18(1), 010501 (2012).

D. Cai, T.T.W. Wong, L. Zhu, J. Shi, S.-L. Chen, and L. V. Wang, Dual-view photoacoustic microscopy for quantitative cell nuclear imaging, Opt. Lett. 43(20), 4875 (2018).

S. Arridge, P. Beard, M. Betcke, et al., Accelerated high-resolution photoacoustic tomography via compressed sensing, Phys. Med. Biol. 61(24), 8908-8940 (2016).

M.P. Mienkina, K. Hensel, T.N. Le, et al., Experimental characterization of ferucarbotran (resovist ()) as a photoacoustic nanoparticle contrast agent, Proc. - IEEE Ultrason. Symp. 1, 393-396 (2006).

W. Mahmood Mat Yunus, K.S. Chan, and W.M. Zin Wan Yunus, Study on photobleaching of methylene blue doped in PMMA, PVA and gelatin using photoacoustic technique, J. Nonlinear Opt. Phys. Mater. 12(01), 91-100 (2003).

N.A. George, B. Aneeshkumar, P. Radhakrishnan, and C.P.G. Vallabhan, Photoacoustic study on photobleaching of Rhodamine 6G doped in poly(methyl methacrylate), J. Phys. D. Appl. Phys. 32(14), 1745-1749 (1999). 
R. Carpentier, R.M. Leblanc, and M. Mimeault, Photoinhibition and chlorophyll photobleaching in immobilized thylakoid membranes, Enzyme Microb. Technol. 9(8), 489-493 (1987).

A.P. Xu, H.H. Han, J. Lu, et al., Charge transfer NIR dyes for improved photoacoustic effect, Dye. Pigment. 125, 392-398 (2016).

S. Park, U. Jung, S. Lee, D. Lee, and C. Kim, Contrast-enhanced dual mode imaging: photoacoustic imaging plus more, Biomed. Eng. Lett. 7(2), 121-133 (2017).

J. Chen, Z. Gao, G. Li, and T.D. Wang, Dual-modal in vivo fluorescence and photoacoustic imaging using a heterodimeric peptide, Chem. Commun. (Camb). 54(94), 13196-13199 (2018).

R. Weissleder, A clearer vision for in vivo imaging, Nat. Biotechnol. 19, 316-317 (2001).

D. Armbruster and T. Pry, Limit of Blank, Limit of Detection and Limit of Quantitation, Clin Biochem Rev 29(August), 49-52 (2008).

J. Joseph, K.N. Baumann, P. Koehler, et al., Distance dependent photoacoustics revealed through DNA nanostructures, Nanoscale 9(42), 16193-16199 (2017).

K. Haedicke, C. Brand, M. Omar, V. Ntziachristos, T. Reiner, and J. Grimm, Sonophore labeled RGD : a targeted contrast agent for optoacoustic imaging, Biochem. Pharmacol. 6, 1-8 (2017).

J. Laufer, A. Jathoul, M. Pule, and P. Beard, In vitro characterization of genetically expressed absorbing proteins using photoacoustic spectroscopy, Biomed. Opt. Express 4(11), 2477 (2013).

J. Joseph, M.R. Tomaszewski, I. Quiros-Gonzalez, J. Weber, J. Brunker, and S.E. Bohndiek, Evaluation of precision in optoacoustic tomography for preclinical imaging in living subjects., J. Nucl. Med. 58(5), 807-814 (2017).

C. Liu, Y. Yang, Z. Qiu, Y. Huang, and L. Sun, In Vivo Assessment of Protease Activity in 
Colorectal Cancer by Using Activatable Molecular Photoacoustic Imaging, 2015 IEEE Int. Ultrason. Symp. 4-7 (2015).

N. Tagaya, R. Yamazaki, A. Nakagawa, et al., Intraoperative identification of sentinel lymph nodes by near-infrared fluorescence imaging in patients with breast cancer, Am. J. Surg. 195(6), 850-853 (2008).

S. Ohnishi, S.J. Lomnes, R.G. Laurence, A. Gogbashian, G. Mariani, and J. V Frangioni, Organic Alternatives to Quantum Dots for Intraoperative Near-Infrared Fluorescent Sentinel Lymph Node Mapping, 4(3), 172-181 (2005).

W.S. Tummers, R.H. Kimura, L. Abou-elkacem, et al., Development and Preclinical Validation of a Cysteine Knottin Peptide Targeting Integrin a v b 6 for Near-infrared Fluorescent-guided Surgery in Pancreatic Cancer, 1667-1677 (2018).

S. Alfonso-Loeches, M. Pascual, and C. Guerri, Gender differences in alcohol-induced neurotoxicity and brain damage, Toxicology 311(1-2), 27-34 (2013).

A. Azhdarinia, P. Ghosh, S. Ghosh, N. Wilganowski, and E.M. Sevick-Muraca, Dual-labeling strategies for nuclear and fluorescence molecular imaging: A review and analysis, Mol. Imaging Biol. 14(3), 261-276 (2012).

M. Dabek, L. Ferrier, A. Annahazi, et al., Intracolonic infusion of fecal supernatants from ulcerative colitis patients triggers altered permeability and inflammation in mice: Role of cathepsin $\mathrm{G}$ and protease-activated receptor-4, Inflamm. Bowel Dis. 17(6), 1409-1414 (2011).

B. Cox, J.G. Laufer, S.R. Arridge, and P.C. Beard, Quantitative spectroscopic photoacoustic imaging: a review, J. Biomed. Opt. 17(6), 061202 (2012).

X.L. Deán-Ben, R. Ma, D. Razansky, and V. Ntziachristos, Statistical approach for optoacoustic 
image reconstruction in the presence of strong acoustic heterogeneities, IEEE Trans. Med. Imaging 30(2), 401-408 (2011).

D. Tholen, M. Kroll, J. Astles, et al., EP06-A Evaluation of the Linearity of Quantitative Measurement Procedures: A Statistical Approach; Approved Guideline (2003).

F.M. Brochu, J. Brunker, J. Joseph, M.R. Tomaszewski, S. Morscher, and S.E. Bohndiek, Towards quantitative evaluation of tissue absorption coefficients using light fluence correction in optoacoustic tomography, IEEE Trans. Med. Imaging 36(1), 322-331 (2017).

L. Feng, D. Tao, Z. Dong, et al., Near-infrared light activation of quenched liposomal Ce6 for synergistic cancer phototherapy with effective skin protection, Biomaterials 127, 13-24 (2017).

A. Bychkov, V. Simonova, V. Zarubin, E. Cherepetskaya, and A. Karabutov, The Progress in Photoacoustic and Laser Ultrasonic Tomographic Imaging for Biomedicine and Industry: A Review, Appl. Sci. 8(10), 1931 (2018).

E. Hemmer, A. Benayas, F. Légaré, and F. Vetrone, Exploiting the biological windows: Current perspectives on fluorescent bioprobes emitting above $1000 \mathrm{~nm}$, Nanoscale Horizons 1(3), 168-184 (2016).

C. Lutzweiler and D. Razansky, Optoacoustic imaging and tomography: Reconstruction approaches and outstanding challenges in image performance and quantification, Sensors (Switzerland) 13(6), 7345-7384 (2013).

140 L.J. Rich and M. Seshadri, Photoacoustic Imaging of Vascular Hemodynamics: Validation with Blood Oxygenation Level-Dependent MR Imaging, Radiology 275(1), 110-118 (2014).

J. Kang, E.K. Kim, G.R. Kim, C. Yoon, T.K. Song, and J.H. Chang, Photoacoustic imaging of breast microcalcifications: A validation study with 3-dimensional ex vivo data and spectrophotometric 
measurement, J. Biophotonics 8(1-2), 71-80 (2015).

J.R. Cook, W. Frey, and S. Emelianov, Quantitative Photoacoustic Imaging of Nanoparticles in Cells and Tissues, ACS Nano 7(2), 1272-1280 (2013).

L. Nie, M. Chen, X. Sun, P. Rong, N. Zheng, and X. Chen, Palladium nanosheets as highly stable and effective contrast agents for in vivo photoacoustic molecular imaging, Nanoscale 6(3), 1271$1276(2014)$.

S.A. Ermilov, T. Khamapirad, A. Conjusteau, et al., Laser optoacoustic imaging system for detection of breast cancer, J. Biomed. Opt. 14(2), 024007 (2009).

S. Manohar, S.E. Vaartjes, J.C.G. van Hespen, et al., Initial results of in vivo non-invasive cancer imaging in the human breast using near-infrared photoacoustics, Opt. Express 15(19), 12277 (2007).

S. Mallidi, K. Watanabe, D. Timerman, D. Schoenfeld, and T. Hasan, Prediction of tumor recurrence and therapy monitoring using ultrasound-guided photoacoustic imaging, Theranostics 5(3), 289$301(2015)$.

A. Bunschoten, T. Buckle, J. Kuil, et al., Targeted non-covalent self-assembled nanoparticles based on human serum albumin, 33(3), 867-875 (2012).

C.A. Davies-Venn, B. Angermiller, N. Wilganowski, et al., Albumin-binding domain conjugate for near-infrared fluorescence lymphatic imaging, Mol. Imaging Biol. 14(3), 301-314 (2012).

Y. Wang, L. Lang, P. Huang, et al., In vivo albumin labeling and lymphatic imaging, Proc. Natl. Acad. Sci. 112(1), 208-213 (2015).

Q. Chen, C. Liang, X. Wang, J. He, Y. Li, and Z. Liu, An albumin-based theranostic nano-agent for dual-modal imaging guided photothermal therapy to inhibit lymphatic metastasis of cancer post 
surgery, Biomaterials 35(34), 9355-9362 (2014).

L.V. Wang, Xueyi Xie, Jung-Taek Oh, et al., Combined Photoacoustic and Molecular Fluorescence Imaging In Vivo, 2005 IEEE Eng. Med. Biol. 27th Annu. Conf. 190-192 (2006). 
C. Kim, K.H. Song, F. Gao, and L. V. Wang, Sentinel Lymph Nodes and Lymphatic Vessels: Noninvasive Dual-Modality in Vivo Mapping by Using Indocyanine Green in Rats-Volumetric Spectroscopic Photoacoustic Imaging and Planar Fluorescence Imaging, Radiology 255(2), 442450 (2010).

V. Saxena, M. Sadoqi, and J. Shao, Degradation Kinetics of Indocyanine Green in Aqueous Solution, J. Pharm. Sci. 92(10), 2090-2097 (2003).

J.T. Alander, I. Kaartinen, A. Laakso, et al., A Review of indocyanine green fluorescent imaging in surgery, Int. J. Biomed. Imaging 2012, (2012).

F. Rotermund, R. Weigand, W. Holzer, M. Wittmann, and A. Penzkofer, Fluorescence spectroscopic analysis of indocyanine green J aggregates in water, J. Photochem. Photobiol. A Chem. 110(1), 7578 (1997).

V. Saxena, M. Sadoqi, and J. Shao, Enhanced photo-stability, thermal-stability and aqueous-stability of indocyanine green in polymeric nanoparticulate systems, J. Photochem. Photobiol. B Biol. 74(1), 29-38 (2004).

W.S. Tummers, S.E. Miller, N.T. Teraphongphom, et al., Intraoperative Pancreatic Cancer Detection using Tumor-Specific Multimodality Molecular Imaging, Ann. Surg. Oncol. 25(7), 18801888 (2018).

S.T. Proulx, Q. Ma, D. Andina, J.-C. Leroux, and M. Detmar, Quantitative measurement of lymphatic function in mice by noninvasive near-infrared imaging of a peripheral vein, JCI Insight 2(1), 362-365 (2017).

166

J. Laufer, A. Jathoul, M. Pule, and P. Beard, In vitro characterization of genetically expressed absorbing proteins using photoacoustic spectroscopy, 4(11), 2477-2490 (2013). 
J. Joseph, K.N. Baumann, P. Koehler, et al., Distance dependent photoacoustics revealed through DNA nanostructures, Nanoscale 9(42), 16193-16199 (2017).

K. Cardinell, N. Gupta, J.C. Kumaradas, et al., Assessing Near-Infrared Dyes for In Vivo Photoacoustic Lymphatic Imaging, Submitted (2019).

Fluorescein, Oregon Green and Rhodamine Green Dyes-Section 1.5, ThermoFisher Sci. (n.d.).

F. Heisig, S. Gollos, S.J. Freudenthal, A. El-Tayeb, J. Iqbal, and C.E. Müller, Synthesis of BODIPY derivatives substituted with various bioconjugatable linker groups: A construction kit for fluorescent labeling of receptor ligands, J. Fluoresc. 24(1), 213-230 (2014).

N. Boens, V. Leen, and W. Dehaen, Fluorescent indicators based on BODIPY, Chem. Soc. Rev. 41(3), 1130-1172 (2012).

X. Kong, F. Su, L. Zhang, et al., A Highly Selective Mitochondria-Targeting Fluorescent K + Sensor, Angew. Chemie - Int. Ed. 54(41), 12053-12057 (2015).

A.B. Nepomnyashchii, M. Bröring, J. Ahrens, and A.J. Bard, Synthesis, photophysical, electrochemical, and electrogenerated chemiluminescence studies. multiple sequential electron transfers in BODIPY monomers, dimers, trimers, and polymer, J. Am. Chem. Soc. 133(22), 8633$8645(2011)$.

K. Hirayama, S. Akashi, M. Furuya, and K. Fukuhara, Rapid confirmation and revision of the primary structure of bovine serum albumin by esims and Frit-FAB LC/MS, Biochem. Biophys. Res. Commun. 173(2), 639-646 (1990).

B.L. Bozaquel-Morais, J.B. Madeira, C.M. Maya-Monteiro, C.A. Masuda, and M. Montero-Lomeli, A new fluorescence-based method identifies protein phosphatases regulating lipid droplet metabolism, PLoS One 5(10), (2010). 
L. Quan, S. Liu, T. Sun, et al., Near-infrared emitting fluorescent BODIPY nanovesicles for in vivo molecular imaging and drug delivery, ACS Appl. Mater. Interfaces 6(18), 16166-16173 (2014).

J. Zhang, L. Wang, and Z. Xie, Size-Tunable and Crystalline BODIPY Nanorods for Bioimaging, ACS Biomater. Sci. Eng. 4(6), 1969-1975 (2018).

M. Albrecht, A. Lippach, M.P. Exner, et al., Site-specific conjugation of 8-ethynyl-BODIPY to a protein by [2+3] cycloaddition, Org. Biomol. Chem. 13(24), 6728-6736 (2015).

J. Karolin, L.B.A. Johansson, L. Strandberg, and T. Ny, Fluorescence and Absorption Spectroscopic Properties of Dipyrrometheneboron Difluoride (BODIPY) Derivatives in Liquids, Lipid Membranes, and Proteins, J. Am. Chem. Soc. 116(17), 7801-7806 (1994).

D. Zhang, L. Vetrivel, and A.S. Verkman, Aquaporin deletion in mice reduces intraocular pressure and aqueous fluid production., J. Gen. Physiol. 119(6), 561-569 (2002). 